\title{
Recommended Documentation for Computer Users
}

\section{at ANL}

By

April A. Heiberger

April 1992 


\section{ACKNOWLEDGMENTS}

Clifford Caruthers and Laura Opitz wrote the previous revision of this document. Mona Beavers, Pete Bertoncini, Fran Carnaghi, Linda Clark, Bob McMahon, Fred Moszur, Gary Schlesselman, and Mike Thommes assisted in the revision and review of this document.

We have created this document with Waterloo Generalized Markup Language (GML) and Waterloo Script (Version 86.1). 


\section{PREFACE}

Recommended Documentation for Computer Users at ANL is for all users of the services available from the Argonne National Laboratory (ANL) Computing and Telecommunications Division (CTD). This document will guide you in selecting available documentation that will best fill your particular needs.

Chapter 1 explains how to use this document to select documents and how to obtain them from the CTD Document Distribution Counter. Chapter 2 contains a table that categorizes available publications. Chapter 3 gives descriptions of the online DOCUMENT command for CMS, the VAX, and the Sun workstation. DOCUMENT allows you to scan for and order documentation that interests you. Chapter 4 lists publications ${ }^{1}$ by subject. Categories I and IX cover publications of a general nature and publications on telecommunications and networks, respectively. Categories II, III, IV, V, VI, VII, VIII, and X cover publications on specific computer systems. Category XI covers publications on advanced scientific computing at Argonne. Chapter 5 contains abstracts for each publication, all arranged alphabetically. Chapter 6 describes additional publications containing bibliographies and master indexes that the user may find useful. The appendix identifies available computer systems, applications, languages, and libraries.

\section{What's New In This Revision}

This revision includes abstracts and references for many documents added to the Document Distribution Counter inventory since the previous revision of this document in February 1990. Abstracts and references for obsolete documents (no longer in stock) have been deleted. Also, this revision includes a chapter on the use of the DOCUMEN'T command in CMS, VMS, and Unix.

\footnotetext{
1 In addition to documents, a small number of computer diskettes are available at the Document Distribution Counter. Chapters 4 and 5 list these diskettes along with the available documents.
}

\section{LImltations on the Use of Proprletary Software}

All Argonne users can use available software without restrictions. However, software vendors prohibit the use of certain software by outside (non-Argonne or non-DOE) users. Usually each outside user (or organization) must obtain permission from the vendor of the software package, and an additional surcharge is frequently required. Software packages in this category include:

$\begin{array}{ll}\text { Cuechart } & \text { Librarian } \\ \text { Disspla } & \text { SAS } \\ \text { Data Connection } & \text { Script } \\ \text { Historian } & \text { Speakeasy } \\ \text { IMSL } & \text { Tellagraf }\end{array}$




\section{CONTENTS}

Acknowledgments $\ldots \ldots \ldots \ldots \ldots \ldots \ldots \ldots \ldots \ldots \ldots \ldots \ldots \ldots$

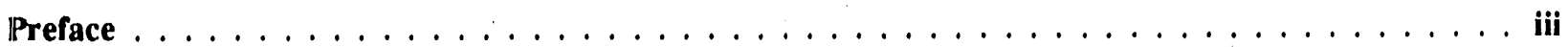

What's New in This Revision $\ldots \ldots \ldots \ldots \ldots \ldots \ldots$ iii

Limitations on the Use of Proprietary Software $\ldots \ldots \ldots \ldots \ldots$. . . . . . . . . . . . .

Chapter 1: Documentation Distribution Practices $\ldots \ldots \ldots \ldots \ldots \ldots \ldots$

How to Select Manuals for Your Needs $\ldots \ldots \ldots \ldots \ldots \ldots \ldots \ldots \ldots$

How to Obtain Publications $\ldots \ldots \ldots \ldots \ldots \ldots \ldots$

Chapter 2: Subject Categories for Publications $\ldots \ldots \ldots \ldots \ldots \ldots \ldots$

Chapter 3: Online Document Programs $\ldots \ldots \ldots \ldots \ldots \ldots \ldots \ldots$

Chapter 4: Recommended Publications by Subject $\ldots \ldots \ldots \ldots \ldots$

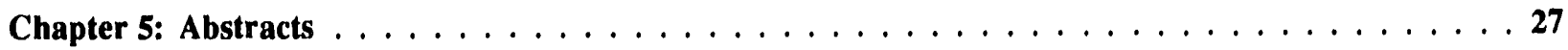

Chapter 6: Bibliographies and Master Indexes $\ldots \ldots \ldots \ldots \ldots$

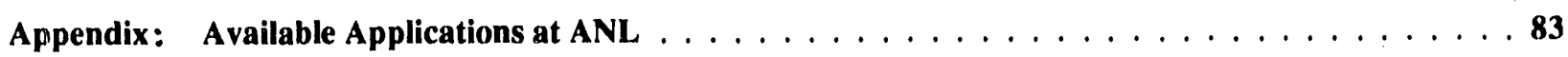

TABLES

1. Organization of Available Publications $\ldots \ldots \ldots \ldots \ldots \ldots \ldots$

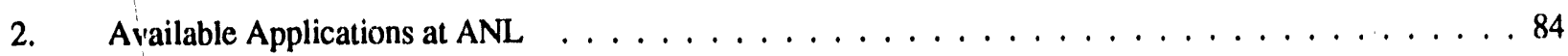




\section{CHAPTER 1}

\section{DOCUMENTATION DISTRIBUTION PRACTICES}

Users of the computing systems at ANL may purchase documents from the CTD Document Distribution Counter (Building 221, Room A-139) or through the mail (by calling extension 2-5405 and ordering them). We stock over 300 documents (including many IBM, $\mathrm{DEC}$, and Cray documents). Vendor documentation is also available for commercial packages used at ANL (such as SAS, Script, Speakeasy, and Tellagraf). We also stock many documents by CTD personnel and Mathematics and Computer Science personnel. These documents (called technical memoranda and ANL reports) usually report on various computational research projects or provide information specific to the ANL computer environment. Although we distribute some software for IBM and Apple Macintosh personal computers, Apple Macintosh software is also available on the Public Volume in AppleTalk zone "Public AlisaTalk" on file server "VAXserver." If you need a binder for a document, some are available from Publix Office Supplies through the Customer Information Control System (CICS).

In addition, we publish the Argonne Computing Newsletter, a monthly newsletter that contains items of topical interest. Users with current computer accounts automatically receive the Argonne Computing Newsletter. To be added to the Newsletter mailing list, call Claudette DaCosse at 708-252-5415. Other sources of more up-to-date information include bulletins (sent to those on the Argonne Computing Newsletter mailing list), online NEWS files, online HELP, sign-on messages, and a tape-recorded system status message that you can hear by calling extension 2-5466.

Between document revisions, CTD informs users of changes in services by means of addenda inserted into documents in stock. We do not automatically notify previ us document purchasers of addenda (although updates are indicated in the Newsletter), but users can keep track of new addenda by invoking the online DOCUMENT e. ec.

\section{HOW TO SELECT MANUALS FOR YOUR NEEDS}

Use Recommended Documentation for Computer Users at ANL to help you select the documentation you should order. In Chapter 2 you will find the subject categories for available publications.

Category I, "General Documentation," is subdivided into "Computer Protection," "Documentation Standards," and "General Applications."

Categories II through VI list applications and other relevant documents for Cray UNICOS (Category II), IBM CMS and MVS batch (Categories III and IV), DEC VAX/VMS (Category V), and Unix services (Category VI). You will find applications and documents for personal computers and scientific workstations in Category VII.

Category VIII covers the "Advanced Computing Research Facility." With two exceptions, these documents are available at no charge only through the Mathematics and Computer Science Division (extension 2-7222).

Category IX, "Telecommunications and Networks," lists documents on telecommunications, clectronic mail, and network access.

Category X, "Management Information Systems," covers the administrative sub-categories of "Disaster Recovery," "Financial Modeling," "Materials Systems," and "MIS Planning."

Category XI, "Computational Research," lists documents on advanced scientific computing at Argonne.

Refer to Table 1 in Chapter 2 to determine the category for a particular topic or computer system in which you are interested. Chapter 3 contains descriptions of the online DOCUMENT command in CMS, 
VMS, and the Unix. If you are looking for a particular application to use with Cray UNICOS, DEC VAX/VMS, IBM CMS, or IBM MVS batch, scan Table 2 in the appendix for the application you want. Look under the appropriate system to find the available softwarc packages.

To see a short abstract of each publication, arranged alphabetically, see Chapter 5. For further information on documentation outside the scope of this document, refer to the bibliographies listed in Chapter 6.

\section{HOW TO OBTAIN PUBLICATIONS}

CTD provides sclected computer-related documentation for Argonne employees or users of Argonne's computer systems. To recover documentation costs, CTD charges a user's account for each document the user receives. The price of each document reflects the cost of purchasing, publishing, producing, and handling. Argonne employees who do not have computer accounts can charge documentation costs to the general overhead account of their organization.

To request documents by telephone, call extension 2-5405. To pick up documents yourself, complete and present the "Documentation Request" form (CTD-107) in person at the service counter in Room A-134. When you need assistance in completing this form or have other related questions, visit the User Services Consulting Office (Room A-139) or call extension 2-5405.

Unless you specify otherwise, we will mail outof-stock documents to you when the shipment arrives.

Currently, if we have an agreement with a vendor (Computer Associates, Cray Research, Digital Equipment Corporation, and IBM), we can place a special order for documents we do not stock. CTD will receive the documents and verify that the order has been filled correctly before mailing them to you. Also, you can order any special document through any Technical Information Services (TIS) library.

Certain documents (technical memoranda, articles, and papers) dealing with the Advanced Computing Research Facility are available at no charge only through the Mathematics and Computer Science Division (extension 2-7222).

If you have any questions about documents, call the User Services consultant on duty at extension 2-5405.

If you are not receiving the Argonne Computing Newsletter but wish to receive it, call the User Services consultant on duty at extension 2-5405. There is no charge for the Argonne Computing Newsletter. 


\section{CHAPTER 2}

\section{SUBJECT CATEGORIES FOR PUBLICATIONS}

For easy reference, we have grouped publications available at the Document Distribution Counter into 11 categories. Categories I and IX cover publications of a general nature and publications on telecommunications and networks, respectively. Categories II, III, IV, V, VI, VII, VIII, and $X$ cover publications on specific computer systems. Category XI covers publications on advanced scientific computing at Argonne. Table 1 lists these categories and sub-categories. If you do not find a document title that describes a specific applica- tion, contact the User Services consultants for assistance.

We recommend that new users enroll in the CTD introductory courses and obtain the documents recommended for those courses. In particular, to get an overview of the computing facilities available at Argonne, we urge you to read Guide to Computing at ANL ANL/TM 336, REVISION 2). 
Table 1

Organization of A vailable Publications

CATEGORY

SUB-CATEGORIES

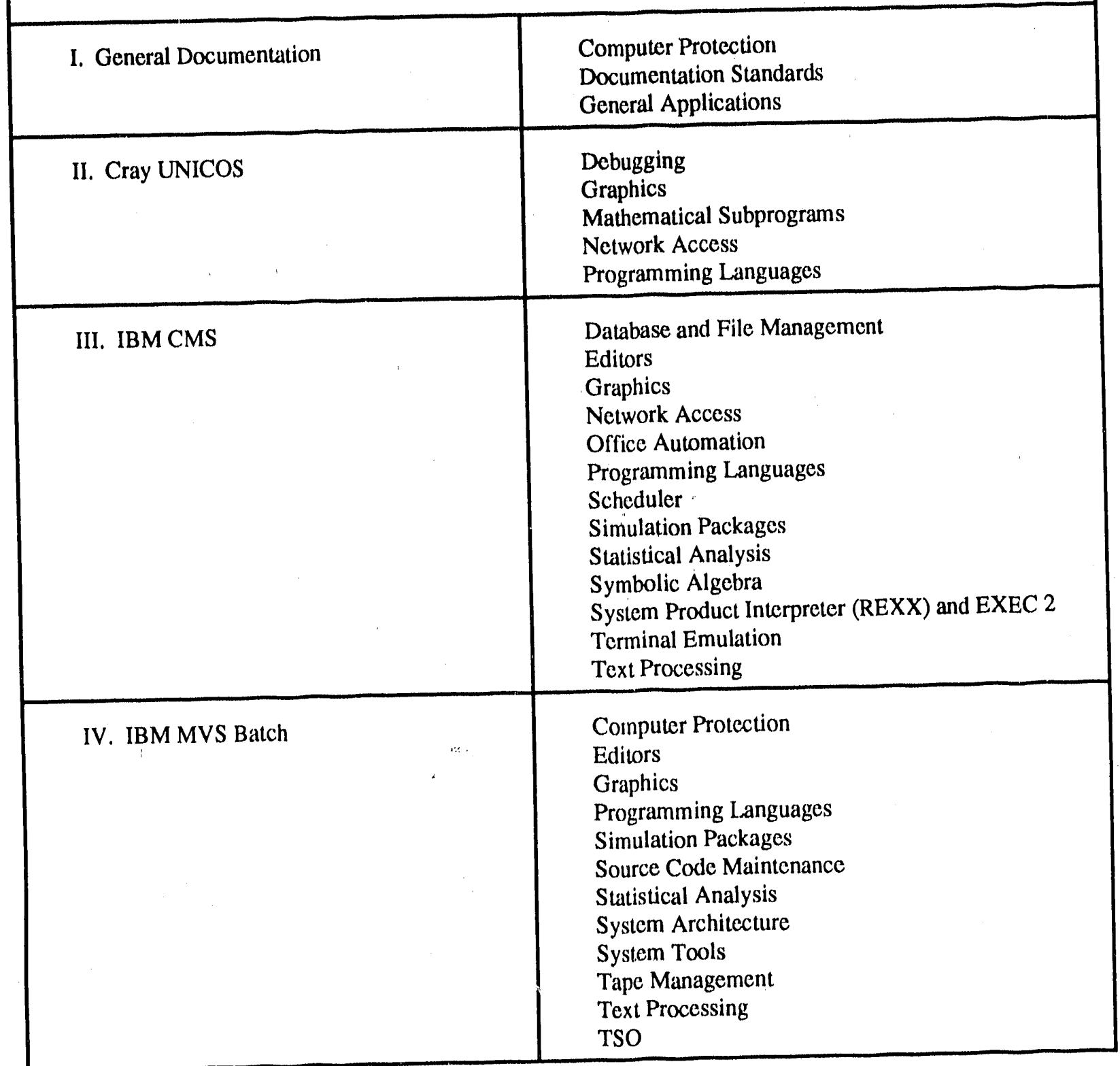


Table 1 (Continued)

Organization of Available Publications

CATEGORY

SUB-CATEGORIES

\begin{tabular}{|c|c|}
\hline V. DEC VAX/VMS & $\begin{array}{l}\text { Debugging } \\
\text { Digital Command Language } \\
\text { Editors } \\
\text { Graphics } \\
\text { Mathematical Libraries } \\
\text { Programming Languages } \\
\text { Statistical Analysis } \\
\text { Symbolic Algebra } \\
\text { Text Processing } \\
\end{array}$ \\
\hline VI. Unix Services & $\begin{array}{l}\text { Editors } \\
\text { Graphics } \\
\text { Parallel Programming } \\
\text { Symbolic Algebra } \\
\end{array}$ \\
\hline $\begin{array}{l}\text { VII. Personal Computers and } \\
\text { Scientific Workstations }\end{array}$ & $\begin{array}{l}\text { Communications } \\
\text { File Transfer } \\
\text { Graphics } \\
\text { Statistical Analysis } \\
\text { Terminal Emulation } \\
\text { Visualization Tools }\end{array}$ \\
\hline \multicolumn{2}{|c|}{ VIII. Advanced Computing Research Facility } \\
\hline IX. Telecommunications and Networks & $\begin{array}{l}\text { BITnet } \\
\text { ESnet } \\
\text { Telephone Services }\end{array}$ \\
\hline X. Management Information Systems & $\begin{array}{l}\text { Disaster Recovery } \\
\text { Financial Modeling } \\
\text { Materials Systems } \\
\text { MIS Planning } \\
\end{array}$ \\
\hline XI. Computational Research & \\
\hline
\end{tabular}




\section{CHAPTER 3 \\ ONLINE DOCUMENT PROGRAMS}

The DOCUMENT program is available in CMS, VAX/VMS, and the central Unix servers to provide online information contained in Recommended Documentation for Computer Users at ANL (ANL/TM 379, REVISION 3). This capability provides users with a convenient way to determine what documents are available for distribution by CTD. The software also provides users the ability to order documents easily. In addition to providing titles, the DOCUMENT program provides price, current date of publication, and the date of the most current addendum (if any). The date field indicates the most current versions in the CTD inventory; the addendum date reflects vendor technical newsletters or locally written addenda that CTD automatically distributes with the current base document.

To request document information or to order documents in CMS or the central VAX cluster, enter:

$$
\text { DOCOMANT topia }
$$

where "topic" is a portion of the title of the document, the document-code number, or a category of documents (such as Fortran). To request document information or to order documents from the achilles Sun server, enter:

\section{documant topic}

where "topic" is a single word with more than one character but less than 20 characters. DOCUMENT will display the document information at your terminal or workstation.

To request a document abstract, enter (in CMS):

DOCUMENT ABSTRACT documant-coda

where "document-code" is a unique code (up to 16 characters) that corresponds to vendor-assigned reference codes. When vendors do not use such codes, CTD arbitrarily generates a unique code from the document title.
To obtain further information on DOCUMENT in CMS or VMS, enter:

HortP DOCUMANT

To obtain further information on DOCUMENT on the achilles Sun server, enter:

man documant

The DOCUMENT program can be installed in other Unix or VMS environments. For additional information, contact the User Services consultants at exten. sion 2-5105.

\section{Online DEC Documentation}

Bookreader, a VMS DECwindows (X Window) application, is an online information display tool that displays final-form files that are optimized for fast online display and rapid random access. Users can view Bookreader documents on the Argonne central VAX cluster from $X$ Window servers. The central VAX cluster provides Bookreader access to the entire VMS documentation set and other DEC software and hardware documentation.

\section{Online Sun Documentation}

Sun Microsystems distributes documents as online documentation available with a product called Answerbook. If you use the Sun environment, contact your division's Sun administrator to learn if Answerbook is available. 


\section{CHAPTER 4}

\section{RECOMMENDED PUBLICATIONS BY SUBJECT}

\section{GENERAL DOCUMENTATION}

A Plan for Scientific Visualizaiton at ANL (ANL/TM 476)

Argonne National Laboratory Computer Networks

Argonne National Laboratory Computing and Telecommunications Division

Argonne National Laboratory Computing and Telecommunications Division Rates

Central IBM Systems at Argonne National Laboratory

Central VAX Computing at Argonne National Laboratory

CMS at ANL (ANL/TM 423, REVISION 2)

Computational Science and Engineering at Argonne National Laboratory

Cray Computing at Argonne National Laboratory

CTD Information Packet

Electronic Mail at ANL (ANL/TM 431, REVISION 2)

Expert Systems Tools and Applications (0-471-83950-7)

Guide to Computing at ANL (ANL/TM 336, REVISION 2)

Guide to Telecommunications at ANL (ANL/TM 422)

MVS Batch Computing and Data Management at ANL (ANL/TM 400, REVISION 1)

Recommended Documentation for Computer Users at ANL (ANL/TM 379, REVISION 3)

Using ihe Central VAX 8700 Computer at ANL (ANL/TM 455, REVISION 2)

\section{COMPUTER PROTECTION}

Data Physician Plus! Computer Virus Protection System

Disinfectant $v 2.8$

Guide to Computer Protection at ANL (ANL/TM 413)

Security Guidelines for Microcomputers and Word Processors (DOE/MA-0181)

Security of Personal Computer Systems: A Management Guide (NBS500-120)

Sensitive Unclassified Computer Security Program Compliance Review Guidelines (DOE/MA-0188/1)

Viruscan/Clean-Up for IBM PC V86B

\section{DOCUMENTATION STANDARDS}

CTD Writing and Editing Standards (ANL/TM 487)

Guideli es for Document Designers (400-78-0043)

Handbook of Technical Writing, Third Edition (0-312-35810-5)

\section{GENERAL APPLICATIONS}

\section{GRAPHICS}

CA-Graphics Connection User Guide, Version 1.0 (RG 04 GC 10UGS)

Choosing the Right Chart

ISSCO-GKS Command Reference Guide

NCSA HDF Calling Interfaces and Utilities, Version 3.1

NCSA Image for the Color Macintosh, Version 3.0

NCSA PC Show for the IBM PC, Version 1.0

NCSA X DataSline for the X Window System, Version 1.0

NCSA X Image for the X Window System, Version 1.0

SAS/GRAPH User's Guide, Version 5 Edition (0-917382-68-4)

Scientific Chart Design: Computer-Generated Charts Using TELL-A-GRAF

VMS DECwindows User's Guide (AA-MG18A-TE)

$X$ Window System User's Guide (0-937175-14-5) 


\section{CUECHART}

CA-Cuechart User Guide, Release 2.1 (RG99CC2001S)

Cuechart Stencil Maker's Guide

DISSPLA

ANL Supplement to the CA-Disspla User's Manual (ANL/TM 467)

CA-Disspla Codebook: Guide to Making CA-Disspla Codebook Masters (QG 99 CB 10MMS)

CA-Disspla Codebook User Guide (RG 99 CB 1001S)

CA-Disspla First Facts, Version 11.0 (QG 99 DS 11FFS)

CA-Disspla Pocket Guide: Version 11.0 (QG 99 DS 11P1S)

CA-Disspla User Manual, Version 11.0 (RG 99 DS 1101S)

\section{SAS/GRAPH}

SAS/GRAPH Guide to Hardware Interfaces, Version 5 Edition (0-917382-69-2)

SAS/GRAPH Software: Introduction, Version 6, First Edition (1-55544-395-8)

SAS/GRAPH Software: Reference, Version 6, First Edition (1-55544-379-6)

SAS/GRAPH User's Guide, Version 5 Edition (0-917382-68-4)

\section{TELLAGRAF}

CA-DATA CONNECTION User Manual, Release 1.0 (RG99DC1001S)

CA-Tellagraf Brief: Bulletin of Release Information on Enhancement Fixes, Version 6.1 (UD 99 TG 610BS)

CA-Tellagraf CGM B.R.I.E.F. Bulletin of Release Information on Enhancements/Fixes, Version 6.1 (UD 99 TG 61CGS)

CA-Tellagraf Command Reference Manual, Release 7.0 (R199TG7001S)

CA-Tellagraf Menus Primer, Release 1.5 (QG 99 TG 15MPS)

CA-Tellagraf Primer (QG 99 TG 10PRS)

CA-Tellagraf User's Manual, Version 6.1 (RG 99 TG 6101S)

Design Ideas Using Tables

Scientific Chart Design: Computer-Generated Charts Using TELL-A-GRAF

Tables Designer's Guide

Tables User's Guide

TellagrafIPinpoint User's Manual (PIN-UG-0985)
TellagraffPinpoint Workstation Reference Card DEC VT241 VT240 Hewlett-Packard 262326472648 (PIN-QR-1085-DEC)

TellagraflPinpoint Workstation Reference Card IBM 3179 G $3270 P C / G 3270 P C / G X$

(PIN-QR-1085-3179)

TellagraffPinpoint Workstation Reference Card IBM 32793270 PC (PIN-QR-1085-3279)

TellagraflPinpoint Workstation Reference Card Tektronix 4010401240134014401540164051 405240544081 (PIN-QR-1085-4010)

TellagraflPinpoint Workstation Reference Card Tektronix 4027 (PIN-QR-1085-4027)

TellagraflPinpoint Workstation Reference Card Tektronix 410441054106 (PIN-QR-0386-4104)

TellagraflPinpoint Workstation Reference Card Tektronix 410641074109 (PIN-QR-1085-4106)

TellagrafiPinpoint Workstation Reference Card Tektronix 4112411341144115411641254128 4129 (PIN-QR-1085-4112)

\section{MATHEMATICAL LIBRARIES}

FUNPACK--A Package of Special Function Subroutines (TM 385)

Guidelines for Using the AMDLIB, IMSL, and NAG Mathematical Software Libraries at ANL (ANL-81-73)

KWIC Index and Cross Reference Charts (TM 261)

LINPACK User's Guide (0-89871-172-X)

Matrix Eigensystem Routines--EISPACK Guide Extension--51 (3-540-08254-9)

Matrix Eigensystem Routines--EISPACK Guide--6 (3-540-06710-8)

Nonlinear Optimization Routines in AMDLIB (TM 297)

Solution of the General Nonlinear Programming Problem with Subroutine VMCON (ANL-80-64)

User Guide for MINPACK-1 (ANL-80-74)

INTERNATIONAL MATHEMATICAL AND STATISTICAL LIBRARY (IMSL)

IMSL Libraries Edition 10.0 Update Guide (8709) IMSL Library Reference Manual

NUMERICAL ALGORITHMS GROUP LIBRARY (NAGLIB)

NAG Fortran Library Introductory Guide Mark 13 (1-85206-046-8)

NAG Fortran Library Introductory Guide Mark 14 (1-85206-054-9)

NAG Fortran Mini Manual Mark 12 (1-85206-033-6) 


\section{STATISTICAL ANALYSIS SYSTEM (SAS)}

Introducing the SAS System, Version 6, First Edition (1-55544-449-0)

SAS Companion for the CMS Environment, Version 6, First Edition (1-55544-399-0)

SAS Companion for the CMS Operating System (1-55544-001-0)

SAS Companion for the MVS Environment, Version 6 , First Edition (1-55544-398-2).

SAS Companion for the VMS Environment, Version 6 , First Edition (1-55544-392-3)

SAS Guide to Macro Processing, Version 6, Second Edition (1-55544-382-6)

SAS Language and Procedures: Introduction, Version 6. First Edition (1-55544-410-5)

SAS Language and Procedures: Syntax, Version 6, First Edition (1-55544-400-8)

SAS Language and Procedures: Usage, Version 6, First Edition (1-55544-371-0)

SAS Language: Reference, Version 6, First Edition (1-55544.381-8)
SAS Procedures Guide, Version 6, Third Edition (1-55544-378-8)

SAS Programmer's Guide for PL/I, Version 5 Edition (0-917382-91-9)

SAS User's Guide: Basics, Version 5 Edition (0-917382-65-X)

SAS User's Guide: Statistics, Version 5 Edition (0-917382-66-8)

SAS/ETS User's Guide, Version 6, First Edition (1-55544-325-7)

SAS/FSP User's Guide, Version 5 Edition (0-917382-67-6)

SAS/GRAPH Guide to Hardware Interfaces, Version 5 Edition (0-917382-69-2)

SAS/GRAPH Software: Introduction, Version 6, First Edition (1-55544-395-8)

SAS/GRAPH Software: Reference, Version 6, First Edition (1-55544-379-6)

SAS/GRAPH User's Guide, Version 5 Edition (0-917382-68-4)

SUGI Supplemental Library User's Guide, Version 5 Edition (1-55544-000-2) 


\section{CRAY UNICOS}

A Practical Guide to UNIX System V (0-8053-0243-3)

ANL Supplement to the UNICOS Primer (ANL/TM 460)

CF77 Compiling System, Volume 1: Fortran Reference Manual (SR-3071 5.0)

CF77 Compiling System, Volume 2: Compiiler Message Manual (SR-3072 5.0)

CF77 Compiling System, Volume 3: Vectorization Guide (SG-3073 5.0)

CF77 Compiling System, Volume 4: Parallel Processing Guide (SG-3074 5.0)

Cray Research, Inc. Silver Binder (I Inch)

Cray Research, Inc. Silver Binder (1.5 Inch)

Cray Research, Inc. Silver Binder (2 Inch)

Cray Research, Inc. Silver Binder (3 Inch)

Cray Research, Inc. Silver Binder Primer (1.5 Inch)

NSPCG (Non-Symmetrix Preconditioned Conjugate Gradient) User's Guide (CNA-216)

Symbolic Machine Instructions Reference Manual (SR-0085)

UNICOS Overview for Users (SG-2052-A)

UNICOS Performance Utilities Reference Manual (SR-2040 6.0)

UNICOS Primer (SG-2010 6.0)

UNICOS Shell and Variable Ready Reference (SQ-2060)

UNICOS Support Tools Guide (SG-2016 6.0)

UNICOS System Calls Reference Manual (SR-2012 5.0)

UNICOS Tape Subsystem User's Guide (SG-2051 6.0)

UNICOS User Commands Ready Reference Manual (SQ-2056 6.0)

UNICOS User Commands Reference Manual (SR-2011 6.0)

UNICOS vi Reference Card (SQ-2054)

UNICOS X Window System Reference Manual (SR-2101 6.0)

\section{DEBUGGING}

UNICOS CDBX Debugger User's Guide (SG-2094 6.0)

UNICOS CDBX Symbolic Debugger Reference Manual (SR-2091 6.0)

UNICOS Symbolic Debugging Package Reference Manual (SR-0112 C)

\section{GRAPHICS}

\section{DISSPLA}

CA-Disspla First Facts, Version 11.0

(QG 99 DS 11FFS)

CA-Disspla Pocket Guide, Version 11.0

(QG 99 DS 11P1S)

CA-Disspla User Manual, Version 11.0

(RG 99 DS 1101S)

See also "Graphics" in Section I.

\section{FORTRAN}

A Guidebook to Fortran on Supercomputers (0)-12-444760-0)

Fortran (CFT) Reference Manual (SR-0009-M)
Fortran 90 Explained (0-19-853772-7)

Getting Started with Fortran 77

\section{MATHEMATICAL SUBPROGRAMS}

Boeing Mathematical Subprograms Library

\section{NETWORK ACCESS}

TCPIIP and OSI Network User's Guide (SG-2009 6.0)

\section{PROGRAMMING LANGUAGES}

CAL Assembler Reference Manual, Version 2

(SR-2003)

Cray C Reference Manual (SR-2024D)

Cray Standard C Programmer's Reference Manual (SR-2074 3.0)

Cray Y-MP, Cray X-MP, and Cray-1 C Library

Reference Manual (SR-0136C)

Segment Loader (SEGLDR) and LD Reference Manual

(SR-0066 6.0)

UNICOS CFT Reference Card (SQ-0022)

UNICOS CFT77 Reference Card (SQ-0138 B)

UPDATE Reference Manual (SR-0013 K) 
Volume 1: UNICOS Fortran Library Reference Manual (SR-2079 6.0)

Volume 3: UNICOS Math and Scientific Libraryi

Reference Manual (SR-2081 6.0) 


\section{IBM CMS}

IBM Virtual Machine/Facility/370: Directory Maintenance Guide for General Users (SC20-1839)

IBM Virtual Machine Facility/370: Glossary and Master Index (GC20-1813)

IBM Virtual Machine Facility/370: Introduction (GC20-1800)

IBM Virtual Machine Library Binder (SX24-5129)

IBM Virtual Machine/Extended Architecture System Product VM/XA SP, Release 1 and Release 2: CMS Primer (SC23-0368-0)

IBM Virtual Machine/Extended Architecture System Product VM/XA SP, Release I and Release 2: CMS Primer Summary of Commands (SC23-0421-0)

IBM Virtual Machine/Extended Architecture System Product VMIXA SP, Release 1 and Release 2: CMS User's Guide (SC23-0356-0)

IBM Virtual Muchine/Extended Architecture System Product VMIXA SP, Release 2: CMS Command Reference (SC23-0354-1)

IBM Virtual Machine/Extended Architecture System Product VM/XA SP, Release 2: CP Command Reference (SC23-0358-1)

IBM Virtual Machine/Extended Architecture System Product VM/XA SP, Release 2: System Messages and Codes Reference (SC23-0376-1)

IBM 3084 Functional Characteristics (GA22-7088-4)

VM/SP: VM/Pass-Through Facility Guide and Reference (SC24-5208)

\section{DATABASE AND FILE MANAGEMENT}

Sec "Statistical Analysis System (SAS)" in Section I.

\section{EDITORS}

IBM Virtual Machine/Extended Archilecture System Product VMIXA SP, Release 1 and Release 2: System Product Editor Command and Macro Reference (SC23-0372-())

IBM Virtual Machine/Extended Architecture System Product VM/XA SP, Release 1 and Release 2: System Product Editor User's Guide (SC23-0373-0)

VM/System Product: Editor Xedit Program Function Key T'emplate for IBM Display Terminals (SX24-5123)

\section{GRAPHICS}

Sec "Graphics" in Section I.

\section{NETWORK ACCESS}

IBM Transmission Control ProtocollInternet Command Reference Manual (GC09-1204-02)

Network Access to CICS: Full Screen Terminal or ASCII Terminal

\section{OFFICE AUTOMATION}

IBM Binder for PROFS Documents (SB30-0480)

IBM Professional Office System Quick Guide (SX20-2396)

Using Line-Mode Support with the Professional Office System (SH20-6802)

Using the Professional Office System (SH20-6797-01)

VM/System Product: Getting Started with the Professional Office System (SH20-5602)

\section{PROGRAMMING LANGUAGES}

\section{ASSEMBLER}

Programmer's Iniroduction to IBM System/ 360 Assembler Language: Student Text (SC20-1646)

BASIC

IBM Basic Application Programming: System Services (SC26-4028)

IBM Basic Language Reference (GC26-4026-2)

IBM Basic Programming Guide (SC26-4027-2)

VS Basic (CMS) Reference Summary (SX28-6386) 
C

American National Standard for Information Systems--.Programming Language -.C (ANSI X3.159-1989)

C Compiler User's Guide for VM/CMS (SC09-1130-01)

IBM C Language Manual (SC09-1128-01)

The Waite Group's New C Primer Plus

(0-672-22687-1)

\section{FORTRAN}

Fortran (CFT) Reference Manual (SR-0009-M) IBM OS Fortran IV (H Extended) Compiler Programiner's Guide (SC28-6852)

\section{FORTRAN 66}

Fortran 66 (Fortran IV) is obsolete but still available at ANL.

\section{FORTRAN OPTIMIZING COMPILER}

IBM System/370 Fortran H Extended Optimization Enhancement Program Description and Operator's Manual (SH20-2100)

\section{FORTRAN 77}

American National Standard Programming Language Fortran (ANSI X3.9-1978)

Getting Started with Fortran 77

IBM VS Fortran Version 2 Linguage and Library Reference, Release 5 (SC26-4221-6)

IBM VS Fortran Version 2 Programming Guide for CMS and MVS, Release 5 (SC26-4222-5)

VS Fortran Version 2 Interactive Debug Guide and Reference, Release 3 (SC26-4223-2)

\section{FORTRAN 90}

Fortran 90 Explained (0-19-853772-7)

\section{PASCAL}

VS/Pascal Application Programmer Guide (SC26-4319)

VS/Pascal Language Reference (SC26-4320)

\section{PL/I}

Class Notes for a PL/I Course (ANL-76-70)

$O S$ and DOS PL/I Language Reference Manual (GC26-3977)

OS PL/I Checkout and Optimizing Compilers: Keywords Reference Summary (SX33-6002)

OS PL/I Checkout and Optimizing Compilers: Terminal Commands and Compiler Options Summary (SX33-6005)

PL/I Primer: Student Text (SC28-6808)

\section{PL/I OPTIMIZING COMPILER}

Operating System PL/I Optimizing Compiler: Messages (SC33-0027)

OS PL/I Optimizing Compiler: CMS User's Guide (SC33-0037)

OS PL/I Optimizing Compiler: Execution Logic (SC33-0025)

OS PL/I Optimizing Compiler: General Information Manual (GC33-0(0)1)

OS PL/I Optimizing Compiler: Programmer's Guide (SC33-0006)

\section{SPEAKEASY}

Highlights of Speakeasy Epsilon Lectures on Speakeasy

\section{SCHEDULER}

VMSCHEDULE User's Manual

\section{SIMULATION PACKAGES}

\section{CONTINUOUS SYSTEMS MODELING PROGRAM (CSMP)}

Continuous System Modeling Program III and Graphic Feature General Information (GH19-7000)

Continuous System Modeling Program III (CSMP III) Operations Guide (SH19-7002)

\section{STATISTICAL ANAL YSIS}

Sce "Statistical Analysis System (SAS)" in Section I.

\section{SYMBOLIC ALGEBRA}

REDUCE User's Manual 


\section{SYSTEM PRODUCT INTERPRL TER (REXX)} AND EXEC 2

IBM Virtual Machine/Extended Architecture System Product VM/XA SP, Release 1 and Release 2: EXEC 2 Reference (SC23-0361-0)

IBM Virtual Machine/Extended Archilecture System Product VM/XA SP, Release 1 and Release 2: System Product Interpreter Reference (SC23-0374-0)

IBM Virtual Machine/Extended Architecture System Product VM/XA SP, Release 1 and Release 2: System Product Interpreter User's Guide (SC23-0375-0)

\section{TERMINAL EMULATION}

Using the Hydra Protocol Converter for IBM Full Screen Terminal Emulation at ANL (ANL/TM 457, REVISION 1)

\section{TEXT PROCESSING}

Using the Apple LaserWriter at ANL (ANL/TM 452)

\section{POSTSCRIPT}

PostScript Language Reference Manual (0-201-18127-4)

PostScript Language Tutorial and Cookbook (0-201-10179-3)

\section{SCRIPT}

INDEXER: A Program to Produce a Quality Index (TM 299)

Script Reference Card

Waterloo Script and a PostScript Printer

Waterloo Script GML User's Guide

Waterloo Script Reference Manual

Waterloo Script User's Guide

Waterloo Script with Formula Processing

Waterloo Syspub User's Guide 


\section{IBM MVS BATCH}

IBM Interactive System Productivity Facility/Program Development Facility Version 2 Release 3 for MVS Edit and Edit Macros (SC34-4121-00)

IBM Interactive System Productivity Facility/Program Development Facility Version 2 Release 3 for MVS Guide (SC34-4118-00)

IBM System/370 Extended Architecture: Principles of Operation (SA22-7085-1)

IBM 3084 Functional Characteristics (GA22-7088-4)

Introduction to Virtual Storage in System/370 Student Text (GR20-4260)

MVS Batch Computing and Data Management at ANL (ANL/TM 400, REVISION 1)

MVS/Extended Architecture JCL Reference (GC28-1352-4)

MVS/Extended Architecture JCL User's Guide (GC28-1351-4)

MVS/Extended Architecture Message Library: JES3 Messages (GC23-0062-4)

MVS/Extended Archilecture Message Library: System Codes (GC28-1157-6)

MVS/Extended Architecture Message Library: System Messages, Volume 1 ADY-IEB (GC28-1376-7)

MVS/Extended Architecture Message Library: System Messages, Volume 2 IEC.ITV (GC28-1377-7)

MVS/Extended Architecture Operations: JES3 Commands (SC23-0063-3)

MVS/System Product Version 2 Release 2 General Information (GC28-1500-3)

MVS/370 Integrated Catalog Administration Access Method Services Reference, Release 1.2 (GC26-4051-2)

MVS/370 JCL Reference (GC28-1350-3)

MVS/370 JCL User's Guide (GC.28-1349-3)

MVS/370 Message Library: System Codes (GC38-1008)

MVS/370 Message Library: System Messages Volume 1 AHL-IEB (GC28-1374-4)

MVS/370 Message Library: System Messages Volume 2 IEC-ISG (GC28-1375)

OS/VS Tape Labels (GC26-3795-3)

OS/VS2 MVS Overview (GC28-0984)

OS/VS2 MVS System Programming Library: Service Aids (GC28-0674)

OS/VS2 System Programming Library: Data Management (GC26-3830-4)

\section{COMPUTER PROTECTION}

Guide to Computer Protection at ANL (ANL/TM 413)

OS/VS2 MVS RACF Messages and Codes

(SC38-1014-10)

OS/VS2 System Programming Library: Data Management (GC26-3830-4)

RACF General Information Manual (GC28-0722-12)

RACF Summary for Central IBM Computers at ANL (ANL/TM 478)

RACF/MVS General User Command Reference Card (SX28-0609-05)

RACF/VM General User Command Reference Card (SX22-0008-2)

Resource Access Control Facility (RACF) Command Language Reference, Version 1, Release 9 (SC28-0733-11)

Security Guidelines for Microcomputers and Word Processors (DOE/MA-0181)

Security of Personal Computer Systems: A Management Guide (NBS500-120)

Sensitive Unclassified Computer Security Program Compliance Review Guidelines (DOE/MA-0188/1)

\section{MISCELLANEOUS UTILITIES AND} CATALOGED PROCEDURES

MVS Batch Utilities for File Management at ANL (ANL/TM 412)

MVS/370 Data Administration Utilities, Release 1.2 (GC26-4065-3)

Producing Microfiche Output at ANL (TM 373)

\section{EDITORS}

OBS Wylbur Reference Manual

SLAC Wylbur Tutorial

Wylbur User Reference Summary

\section{GRAPHICS}

See "Graphics" in Section I. 


\section{PROGRAMMING LANGUAGES}

\section{ASSEMBLER}

IBM System/370: Principles of Operation (GA22-7000)

IBM System/370: Reference Summary (GX20-1850)

Programmer's Introduction to IBM System/360 Assembler Language: Student Text (SC20-1646)

\section{ASSEMBLER (VS ASSEMBLER)}

OS/VS DOS/VS and VM/370 Assembler Language (GC33-4010)

OS/VS VM/370 Assembler Programmer's Guide (GC33-4021)

\section{COBOL}

IBM OS/VS Cobol Compiler and Library

Programmer's Guide (SC28-6483-2)

IBM VS Cobol for OS/VS (GC26-3857-04)

\section{FORTRAN}

Fortran (CFT) Reference Manual (SR-0009-M) IBM OS Fortran IV ( $H$ Extended) Compiler

Programmer's Guide (SC28-6852)

\section{FOR'TRAN 66}

Fortran 66 (Fortran IV) is obsolcte but still available at ANL.

\section{FORTRAN OPTIMIZING COMPILER}

IBM System/370 Fortran H Extended Optimization Enhancement Program Description and Operator's Manual (SH20-2100)

\section{FORTRAN 77}

American National Standard Programming Language Fortran (ANSI X3.9-1978)

Getting Started with Fortran 77

\section{FORTRAN 90}

Fortran 90 Explained (0-19-853772-7)

\section{PL/I}

Class Notes for a PL/I Course (ANL-76-70)

OS and DOS PL/I Language Reference Manual (GC26-3977)

OS PL/I Checkout and Optimizing Compilers: Keywords Referense Summary (SX33-6()()2)

OS PL/l Checkout and Optimizing Compilers: Terminal Commands and Compiler Options Summary (SX33-6005)

PL/I Primer: Student Text (SC28-6808)

\section{PL/I OPTIMIZING COMPILER}

Operating System PL/I Optimizing Compiler: Messages (SC33-0027)

OS PL/I Optimizing Compiler: Execution Logic. (SC33-()25)

OS PL/I Optimizing Compiler: General Information Manual (GC33-(0)(01)

OS PL/I Optimizing Compiler: Programmer's Guide (SC33-0006)

\section{SIMULATION PACKAGES}

\section{CONTINUOUS SYSTEMS MODELING PROGRAM (CSMP)}

Continuous System Modeling Program III and Graphis. Feature General Information (GH19-7000)

Continuous System Modeling "'ogram III (CSMP III) Operations Guide ('sH19-7002)

TRANSIENT HEAT TRANSFER, VERSION B (THTB)

THTB al ANL (TM 281)

\section{SOURCE CODE MAINTENANCE}

\section{HISTORIAN}

Historian Plus User's Manual

\section{STATISTICAL ANALYSIS}

\section{BIOMEDICAL COMPUTER PROGRAMS (BMDP)}

BMDP Statistical Software Manual (0-520-07112-3) BMDP User's Digest (0-520-(071158)

See also "Statistical Analysis System (SAS)" in Section I. 


\section{SYSTEM ARCHITECTURE}

\section{DIRECT ACCESS DISK DEVICES}

IBM 3380 Direct Access Storage Reference Summary Card (GX26-1678)

Introduction to IBM Direct-Access Storage Devices and Organization Methods (GC20-1649)

\section{PRINTERS}

Fiorms Design Reference Guide for the IBM 3800 Printing Subsystem (GA26-1633)

IBM 3800 Printing Subsystem Character Set Catalog (GA26-1656)

IBM 3800 Printing Subsystem Operator's Guide (GA26-1634)

IBM 3800 Printing Subsystem Programmer's Guide (Gr.26-3846)

Introducing the IBM 3800 Printing Subsystem and Its Programuning (GC26-3829)

Reference Manual for the IBM 3800 Printing Subsystem (GA26-1635)

3800 Printing Subsystem Character Gauge (GX35-5012)

\section{SYSTEM TOOLS}

JES3

Introduction to JES3 (GC28-0607)

JES3 Commands (SC23-0045)

JES3 Commands: Reference Summary (SX23-0007)

JES3 Messages (GC23-0044)

LINKAGE EDI'T OR AND LOADER

MVS/370 Linkage Editor and Loader User's Guide (GC26-4061)

\section{SORTING AND MERGING}

DFSORT Application Programming Guide, Release 11 (SC33-4035-14)

\section{SYSTEM INTERNALS}

IBM System/370: Principles of Operation

(GA22-7000)

IBM System/370: Reference Summary (GX20-1850)
MVS/370 Data Administration Macro Instruction Reference (GC26-4057)

OS/VS2 MVS Supervisor Services and Macro Instructions (GC28-1114)

\section{VIRTUAL STORAGE ACCESS METHOD (VSAM)}

MVS/370 VSAM Administration Guide (GC26-4066) VSAM Primer and Reference (G320-5774)

\section{TAPE MANAGEMENT}

IBM 3803 Model 1, IBM 3420 Models 3, 5, and 7 Magnetic Tape Subsystems Component Description (GA32-(0)20)

IBM 3803 Model 2, IBM 3420 Models 4, 6, and 8 Magnetic Tape Subsystems Component Description (GA32-()(21)

MVS Batch Computing and Data Management at ANL (ANL/TM 4(X), REVISION 1)

\section{TEXT PROCESSING}

Using the Apple LaserWriter at ANL (ANL/TM 452)

\section{POSTSCRIPT}

PostScript Language Reference Manual (0-201-18127-4)

PostScript Language Tutorial and Cookbook $(0-201-10179-3)$

\section{SCRIPT}

INDEXER: A Program to Produce a Quality Index (TM 299)

Script Reference Card

Waterloo Script and a PostScript Printer

Waterloo Script GML User's Guide

Waterloo Script Reference Manual

Waterloo Script User's Guide

Waterloo Script with Formula Processing

Waterloo Syspub User's Guide

\section{TSO}

IBM TSO Extensions Version 2 Command Reference (SC28-1881-3)

IBM TSO Extensions Version 2 User's Guide (SC28-1880)-2)

TSO Messages (GC28-131()-4) 


\section{DEC VAX/VMS}

A Practical Guide to UNIX System V (0-8053-0243-3)

DM User's Guide

Guide to VMS Files and Devices (AA-LA06A-TE)

Pathworks for DOS User's Handbook, Version 4.0 (AA-PAF7B-T'K)

Summary of ANSYS Revision 4.4A New Features

VAX Documentation Binder (1.5 Inch) (99-07858-01)

VAX Documentation Binder (2.5 Inch) (99-07862-01)

VMS User's Manual, Version 5.4 (AA-LA98B-TE)

\section{DEBUGGING}

VMS Debugger Manual (AA-LA59B-TE)

DIGITAL COMMAND LANGUAGE

Guide to Using VMS Command Procedures

(AA-LA11A-TE)

VMS DCL Dictionary: Part I, Version 5.4

(AA-PBK5A-TE)

VMS DCL Dictionary: Part II, Version 5.4

(AA-PBK6A-TE)

\section{EDITORS}

VAX EDT Reference Manual (AA-LA16A-TE)

GRAPHICS

Sce "Graphics" in Strtion I.

MATHEMATICAL LIBRARIES

Sec "Mathematical Lilpraries" in Section I.

PROGRAMMING LANGUAGES

VAX Fortran Languasg Reference Manual

(AA-D034E-TE)

VAX Fortran User's Marmal (AA-D035E-TE)

C

Portable Programs for Parallel Processors (0-03-014404-3)

\section{FORTRAN}

Fortran (CFT) Reference Manual (SR-0009-M)

\section{FORTRAN 77}

American National Standard Programming Language Fortran (ANSI X3.9-1978)

Getting Started with Fortran 77

FORTRAN 90

Fortran 90 Explained (0-19-853772-7)

\section{PROLOG}

An Introduction to the Design, Creation and Use of Dalabases Using Prolog

Prolog Programming for Artificial Intelligence, Second Edition (0-201-416(6-9)

\section{STATISTICAL ANALYSIS}

Sec "Statistical Analysis System (SAS)" in Section I.

\section{SYMBOLIC ALGEBRA}

Introductory MACSYMA Documentation: A Collection of Papers

MACSYMA Reference Manual Volume I

MACSYMA Reference Manual Volume II

\section{TEXT PROCESSING}

\section{LATEX}

La'Tex User's Guide and Reference Manual (0-201-15790-X)

\section{MASS-11}

Mass-11 Word Processing

TEX

The TeXbook (0-201-13448-9) 
A Practical Guide to UNIX System V (0-8053-0243-3)

\section{EDITORS}

Gnu Emacs Manual

Gnu Emacs Reference Card

\section{GRAPHICS}

$X$ Window System User's Guide (0-937175-14-5), Part Threc

See also "Graphics" in Section I.

\section{PARALLEL PROGRAMMING}

Portable Programs for Parallel Processors (0-03-014404-3)

\section{SYMBOLIC ALGEBRA}

Mathematica: A System for Doing Mathematics by Computer (0-201-51507-5) 


\section{PERSONAL COMPUTERS AND SCIENTIFIC WORKSTATIONS}

Microsoft Learning DOS (089-015V200-3.5)

Microsoft Learning DOS (089-015V200-5.25)

Security of Personal Computer Systems: A Management Guide (NBS500-120)

University of Chicago Agreements with Personal Computer Vendors

Using MS-DOS Kermit: Connecting Your PC to the Electronic World (1-55558-082-3)

Using MS-DOS 5 (0-88022-668-4)

\section{COMMUNICATIONS}

\section{APPLE MACINTOSH COMPUTERS}

Network Access to CICS: Apple Macintosh

IBM PERSONAL COMPUTERS

Network Access to CICS: IBM Personal Computer

\section{FILE TRANSFER}

Kermit User Guide

\section{IBM PERSONAL COMPUTERS}

Kermit-MS for IBM Personal Computer, Version 3.II (3 1/2-inch diskette)

Kermit-MS for IBM Personal Computer, Version 3.11 (5 1/4-inch diskette)

\section{GRAPHICS}

See "Graphics" in Section I.

\section{STATISTICAL ANALYSIS}

\section{STATISTICAL ANALySIS SYSTEM (SAS)}

Changes and Enhancements to Base SAS Software for Personal Computers, Release 6.03, SAS Technical Report P-171 (1-55544-071-1)

SAS Guide to the Micro-to-Host Link, Version 6 Edition (1-55544-069-X)

SAS Introductory Guide for Personal Computers, Release 6.03 (1-55544-095-9)

SAS Language and Procedures: Introduction, Version 6, First Edition (1-55544-410-5)

SAS Language and Procedures: Syntax, Version 6, First Edition (1-55544-400-8)
SAS Language Guide for Personal Computers, Release 6.03 (1-55544-099-1)

SAS Procedures Guide for Persona! Computers, Release 6.03 (1-55544-089-4)

SAS Technical Report P-201: Changes and Enhancements to SAS Sofitware for Personal Computers, Release 6.04 (1-55544-393-1)

SAS Technical Report P-204: SAS Software Changes and Enhancements, Release 6.06 (1-55544-409-1)

SAS/ETS User's Guide, Version 6, First Edition (1-55544-325-7)

SAS/PC Base Software, Release 6.04

SAS/PC ETS Software, Release 6.04

SAS/PC Graph Software, Release 6.04

SASIPC Stat Software, Release 6.04

SAS/STAT User's Guide, Release 6.03 Edition (1-55544-088-6)

SAS/STAT User's Guide, Version 6, Fourth Edition (1-55544-376-1)

\section{TERMINAL EMULATION}

NCSA Telnet for PC Version $2.2 T N$ and Version $2.2 D$ (UD-99 US-14)

NCSA Telnet for the IBM PC Version 2.2TN and 2.2D

(3 1/2-inch diskette)

NCSA Telnet for the IBM PC Version $2.27 \mathrm{~N}$ and $2.2 \mathrm{D}$

(5 1/4-inch diskettc)

NCSA Telnet for the Macintosh, Version 2.4

in3270 for the Macintosh Reference Guide, Version 2.0

\section{VISUALIZATION TOOLS}

\section{APPLE MACINTOSH COMPUTERS}

NCSA Image for the Color Macintosh, Version 3.0

IBM PERSONAL COMPUTERS

NCSA PC Show for the IBM PC, Version 1.0

NCSA PC Show for the IBM PC, XT, AT, AND PS/2

User's Guide 


\section{ADVANCED COMPUTING RESEARCH FACILITY (ACRF)}

Each year the Mathematics and Computer Science Division (MCS) produces a complete annotated listing of its publications. These publications, as well as the listing, are available directly from MCS (extension 2-7222).

The documents cited below are selections from the listing that contain information of general interest to Argonne scientists.

Activities and Operations of the Advanced Computing Research Facility, November 1987-December 1988 (ANL-89/1)

Advanced Architecture Computers (ANL/MCS-TM-57, REVISION 2)

DO Loop Transforming Tools

Graphics Tools for Developing High-Performance Algorithms (MCS-P28-1288)

LAPACK Working Note No. 6: Tools to Aid in the Analysis of Memory Access Patterns for Fortran Programs (ANL/MCS-TM-120)

Parallelizing the SDI Access Algorithm for the Connection Machine-2 (ANL-89/41)

The Advanced Computing Research Facility: Opening New Avenues of Advanced Scientific Computing Research 


\section{TELECOMMUNICATIONS AND NETWORKS}

Distribution of Mathematical Software via Electronic Mail (ANL/MCS-TM-48)

Electronic Mail at ANL (ANL/TM 431, REVISION 2)

Guide to Telecommunications at ANL (ANL/TM 422)

Guide to VAX/VMS NJE Networking at ANL (ANL/TM 444)

Investigation of GOSIP Technology at ANL (ANL/TM 499)

Kermit User Guide

Kermit-MS for IBM Personal Computer, Version 3.11 (3 1/2-inch diskette)

Kermit-MS for IBM Personal Computer, Version 3.11 (5 1/4-inch diskette)

Manager's Guide to the Argonne NJE Emulator (ANL/TM 479)

MultiNet Version 3.0 Manuals

NCSA Telnet for PC Version 2.2TN and Version 2.2D (UD-99 US-14)

NCSA Telnet for the IBM PC Version 2.2TN and 2.2D (3 1/2-inch disketue)

NCSA Telnet for the IBM PC Version 2.2TN and 2.2D (5 1/4-inch diskette)

NCSA Telnet for the Macintosh, Version 2.4

Reference Card for Using the Computer Callback Service

Specifications for the PBX-Based Digital Telecommunications System (ANL/TM 418)

Telebit T2500 Asynchronous Modem User's Guide with Operating Instructions (ANL/TM 492)

tn3270 for the Macintosh Reference Guide, Version 2.0

\section{BITNET}

Electronic Mail at ANL (ANL/TM 431, REVISION 2)

\section{ESNET}

The ANL/ESnet Node Maintenance Guide

(ANL/TM 491)

\section{TELEPHONE SERVICES}

Asynchronous Data Communications User Guide with ADI/ACI 100 Operating Instructions (ANL/TM

469)

ITE 4 Telephone User Guide (ANL/TM 471)

ITE 12 Telephone User Guide (ANL/TM 472)

ITE 24 Telephone User Guide (ANL/TM 473)

Single Line Telephone (STE) User Guide (ANL/TM 470) 


\section{MANAGEMENT INFORMATION SYSTEMS}

A Plan for Administrative Computing at ANL FY1989 through FY1991 (ANL/TM 464)

A Plan for Administrative Computing at ANL FY1990 through FY1992 (ANL/TM 474)

A Plan for Administrative Computing at ANL FY1991 through FY1993 (ANL/TM 483)

A Plan for Administrative Computing at ANL FY992 through FY1994 (ANL/TM 489)

\section{DISASTER RECOVERY}

Disaster Recovery Plan for Administrative Systems (ANL/TM 456, REVISION 1)

\section{FINANCIAL MODELING}

Automated Materials/Payables System Information Guide (ANL/TM 481)

Guide to Reporting Time in the Financial Information System at ANL ANL/TM 414)

Manual Purchase Order Closing (ANL/TM 480, REVISION 1)

Overview of the Non-Programmatic Cost Projection Prototype (ANL/TM 440)

Retrieving and Analyzing Automated Materials/Payables System Data at ANL (TM 408)

Retrieving and Analyzing Computer Usage Accounting Data at ANL (ANL/TM 402, REVISION 1)

Using the Financial Management System at ANL (ANL/TM 446)

Using the Purchase Information Reporting System at ANL (ANL/TM 419)

\section{MATERIALS SYSTEMS}

Argonne Materials Order System Requester's Guide Introduction to Materials and Services Tracking at ANL (ANL/TM 448)
Reference Card for the Online Materials Catalog Stores Standing Orders at ANL (ANL/TM 441, REVISION 1)

The Argonne Materials Order System (ANL/TM 454)

\section{MIS PLANNING}

A Plan for Administrative Computing at ANL FY1989 through FY1991 (ANL/TM 464)

A Plan for Administrative Computing at ANL FY1990 through FY1992 (ANL/TM 474)

A Plan for Administrative Computing at ANL FY199I through FY1993 (ANL/TM 483)

A Plan for Administrative Computing at ANL FY992 through FY1994 (ANL/TM 489)

ANL Site Response for the DOE FY1993 Information Technology Resources Long-Range Plan (ANL/TM 485)

ANL Statement of Site Strategy for Computing Workstations (ANL/TM 458, REVISION 3)

CTD Software Library Maintenance Tools for MIS Software (ANL/TM 468)

Survey of ANL Organization Plans for Word Processors, Personal Comiputers, Workstations, and Associated Software (ANL/TM 459, REVISION 3)

The Computing and Telecommunications Division Software Library (ANL/TM 463)

Using System Transmittals at ANL (ANL/TM 437) 


\section{COMPUTATIONAL RESEARCH}

An Assessment of Scientific Computing Technology 1991-2001 (ANL/TM 486)

Argonne National Laboratory Study of the Transfer of Federal Computational Technology to Manufacturing Industry in the State of Michigan (ANL/TM 498)

Center for Energy Research Computation Project Report for FY1988 (ANL/TM 465)

The Advanced Software Development and Commercialization Project: Progress Report PR-1 (ANL/TM 484)

The Advanced Software Development and Commercialization Project: Progress Report PR-2 (ANL/TM 488)

The CERC Report: Results of the CERC Project with Recommendations for ANL Institutional Strategies in Advanced Scientific Computing (ANL/TM 475) 


\section{CHAPTER 5}

\section{ABSTRACTS}

All doc|ıments appear alphabetically by document title. Leading "A's," "An's," and "The's" are ignored.

\section{AC'TIVITIES AND OPERATIONS OF THE ADVANCED COMPUTING RESEARCH FACILITY, NOVEMBER 1987-DECEMBER 1988 (ANL-89/1)}

Describes advanced scientific computing research in the Advanced Computing Research Facility (ACRF), including the projects and proposals of outside users, from November 1987 through December 1988. This document is available through the Mathematics and Computer Sciennce Division (extension 2-7222).

\section{ADVANCED ARCHITEC'TURE COMPUTERS (ANL/MCS-TM-57, REVISION 2)}

Describes the characteristics of several recent computers that employ vectorization or parallelism to achieve high performance in floating-point calculations. The authors consider both top-of-the-range supercomputers and computers based on readily available and inexpensive units. In each case, the document revicws the architectural base, novel features, performance, and cost. This document is available through the Mathematics and Computer Science Division (extension 2-7222).

\section{THE ADVANCED COMPUTING RESEARCH FACILITY: OPENING NEW AVENUES OF ADVANCED SCIENTIFIC COMPUTING RESEARCH}

Is a brochure that briefly defines the Advanced Computing Research Facility (ACRF) and its objectives: (1) to encourage experimentation on computers with innovative designs, (2) to assess the suitability and diverse machines for specific applications, (3) to support the Division's research in parallel computation, and
(4) to operate as a national user facility. This brochure supersedes ACRF: Opening New Avenues of Advanced Scientific Computing Research.

\section{THE ADVANCED SOFTWARE DEVELOPMENT AND COMMERCIALIZATION PROJECT: PROGRESS REPORT PR-1 (ANL/TM 484)}

Is the first of a series of reports pertaining to progress in the Advanced Software Development and Commercialization Project, a joint collaborative effort between the Center for Supercomputing Research and Development of the University of Illinois and the Computing and Telecommunications Division of Argonne National Laboratory. The purpose of this work is to apply techniques of parallel computing that were pioneered by University of Illinois researchers to mature computational fluid dynamics (CFD) and structural dynamics (SD) computer codes developed at Argonne.

\section{THE ADVANCED SOFTWARE DEVELOPMENT AND COMMERCIALIZATION PROJECT: PROGRESS REPORT PR-2 (ANL/TM 488)}

Is the second of a series of reports pertaining to progress in the Advanced Software Development and Commercialization Project, a collaborative effort between the Center for Supercomputing Research and Development of the University of Illinois and CTD. The purpose of this work is to apply techniques of parallel computing, many of which were pioneered by University of Illinois researchers, to mature computational fluid dynamics (CFD) and structural dynamics (SD) computer codes developed at Argonne. 


\section{AMERICAN NA'TIONAL STANDARD FOR INFORMATION SYSTEMS-.PROGRAMMING LANGUAGE-.C (ANSI X3.159-1989)}

Specifies the form and establishes the interpretation of programs expressed in the programming language $C$. Its purpose is to promote portability, reliability, maintainability, and efficicnt exccution of $\mathrm{C}$ language programs on a varicty of computing systems. Sections detail the $\mathrm{C}$ language itself and the contents of the C-language execution library. Appendixes surnmarize aspects of both of them and cnumerate factors that influence the portability of $\mathrm{C}$ programs. This document is intended to guide knowledgeable C-language programmers and implementators of C-language translation systems.

\section{AMERICAN NATIONAL STANDARD PROGRAMMING LANGUAGE FORTRAN (ANSI X3.9-1978)}

Specifies the form and establishes the interpretation of Fortran programs. It consists of a full language and a subset languagc. Its purpose is to promote portability of Fortran programs for use on a variety of data processing systems. The designation "Fortran 77" distinguishes this standard from previous Fortran standards and any possible future revisions. Fortran 77 is a revision of American National Standard Fortran (ANSI X3.9-1966), Fortran is the full language and appears on the right-hand pages; Subset Fortran is a subset of the full language and appears on the left-hand pages.

\section{ANL SITE RESPONSE FOR THE DOE FY 1993 INFORMATION TECHNOLOGY RESOURCES LONG-RANGE PLAN (ANL/TM 485)}

Is one of many contributions to the DOE information tcchnology resources long-range planning process. It provides data on these resources over an eight year period consisting of the base year (FY1990), the current year (FY 1991), the budget year (FY 1992), the plan year (FY 1993), and the out years (FY 1994-FY1997). This dxcument consists of four parts: Part 1, "Site Overview," describes the A I,L Mission, overall organization structure, the strategic approach toward meeting information technology resource needs, the planning process, major issues, and points of contact. Part 2A, "Information Systems Plans for DOE Contractors," defines the current and planned automated information systems associated with the management of ANL, the stewardship of its resources, and the provision of day" to-day general operations and services. Part 3, "Computing Resources Plan," defines the requirements, resources, acquisitions, and budget for computing at ANL for FY1990 through FY1997. Part 4, "Telecommunications Plan," documents the existing and planned telecommunications resources required at ANL from FY1990 through FY1997. Part 5, "Printing and Publishing Plan," updates the ANL Printing and Publishing Activities Plan (FY1992-FY1994) and contains the FY1990 Printing Activities Reports.

\section{ANL STATEMENT OF SITE STRATEGY FOR COMPUTING WORKSTATIONS (ANL/TM 458, REVISION 3)}

Explains the overall Argonne strategy for defining, acquiring, using, and evaluating computing workstations and other automated office support systems. While the primary concern of this document is to ensure optimal integration of computing workstations in the hierarchy of computing that exists at ANL, it also sets forth guidelines for the controlled introduction of other microprocessor technologies that become available in the computing workstation environment. This document supersedes ANL Statement of Site Strategy for Computing Workstations (ANL/TM 458, REVISION 2).

\section{ANL SUPPLEMENT TO THE CA-DISSPLA USER'S MANUAL (ANL/TM 46"7)}

Summarizes installation-dependent options and features of the Computer Associates (CA) Disspla graphics package. 'This document supplements the $C A$ Disspla User Manual. Unless this document states otherwise, you should follow instructions in the $C A$. Disspla User Manual for writing Disspla programs. Information in this document applies to Version 11.0 of Disspla, which is currently installed in CMS, in MVS batch on the central VAX cluster, and in scveral Argonne VAX/VMS systems, and to Version 11.0 of Disspla, which is currently installed on the Cray $\mathrm{X}-\mathrm{MP} / 18$ under the Unix-Based Cray Operating System (UNICOS). This document supersedes all Disspla information in Using Cuechart, Tellagraf, and Disspla at ANL (ANL/TM 433). 


\section{ANL SUPPLEMENT TO THE UNICOS PRIMER (ANL/TM 460)}

Introduces the Cray X-MP interactive and batch services available at Argonne. It serves as a companion to the UNICOS Primer (SG-2010 6.0). Whereas the UNICOS Primer discusses standard Unix issues of Cray computing, this document discusses those issues specific to Cray computing at ANL.

\section{THE ANL/ESNET NODE MAINTENANCE GUIDE (ANL/TM 491)}

Describes the operation and maintenance of the Argonne National Laboratory (ANL) Encrgy Sciences network (ESnet) node. Although this document is written primarily for the CTD Service Engineering Group, others participating in the operation and management of the ANL/ESnet node may also benefit from it.

\section{THE ARGONNE MATERIALS ORDER SYSTEM (ANL/TM 454)}

Describes the procedures and controls implemented by Argonne National Laboratory for systems contracting. Chapter One (the executive summary) lists the organizations directly responsible for the operation of the Argonne Materials Order System (AMOS) and gives a broad overview of the functions of the system. Chapter Two describes in detail how the system works, including internal controls. The appendixes contain facsimiles of relevant forms and topics.

\section{ARGONNE MATERIALS ORDER SYSTEM REQUESTER'S GUIDE}

Is a four-page handout that provides some background on AMOS, explains how to place an AMOS order, and specifies what you must do when you receive your order.

\section{ARGONNE NATIONAL LABORATORY COMPUTER NETWORKS}

Is a brochure that describes the computer networks available at Argonne.

\section{ARGONNE NATIONAL LABORATORY COMPUTING AND TELECOMMUNICATIONS DIVISION}

Is a brochure that provides an overview of the computing services available at Argonne. This brochure supersedes the October 1990 brochure.

\section{ARGONNE NATIONAL LABORATORY COMPUTING AND TELECOMMUNICATIONS DIVISION RATES}

Lists the processing rates of various computers as well as provides information about the computing services, batch services, and interactive services. Computing rates are determined by the shift in effect (primc, overnight, or weekend) when the job starts.

\section{ARGONNE NATIONAL LABORATORY STUDY OF THE TRANSFER OF FEDERAL COMPUTATIONAL TECHNOLOGY TO MANUFACTURING INDUSTRY IN THE STATE OF MICHIGAN (ANL/TM 498)}

Describes a pilot study to develop, initiate the implementation, and document a process to identify computational technology capabilities resident within Argonne National Laboratory to small and mediumsized businesses in the State of Michigan.

\section{AN ASSESSMENT OF SCIENTIFIC COMPUTING TECHNOLOGY 1991-2001 (ANL/TM 486)}

Was originally published as Appendix C ("Information Technology Resources Assessment") of the FY1991-FY1995 Department of Energy (DOE) Information Technology Resources Long-Range Plan. This technical memorandum summarizes the current status and forecasts future trends of the technologies and methods on which scientific computing and the associated information systems are based. DOE uses these findings as guidelines in identifying required technological and human resources and in planning various DOE programs and facilitics. 


\section{ASYNCHRONOUS DATA COMMUNICATIONS \\ USER GUIDE WITH ADI/ACI 100 OPERATING INSTRUCTIONS (ANL/TM 469)}

Describes how to use the Private Branch Exchange (PBX) for asynchronous data communications. This document begins with a general description of the data communication capabilitics of the PBX. The description is fairly detailed; parts of it may ise beyond the interest of casual users who typically use one computer to communicate with another computer system. However, we recommend that all users read this document to gain a fuller understanding of the system.

\section{AUTOMATED MATERIALS/PAYABLES SYSTEM INFORMATION GUIDE (ANL/TM 481)}

Contains general information about the Automated Materials Payables System (AMPS). Management Information Systems (MIS) recommends it for all users of AMPS.

\section{BMDP STATISTICAL SOFTWARE MANUAL $(0-520-07112-3)$}

Is a two-volume set that describes the capabilities and usage of the Biomedical Computer Programs (BMDP). This document covers the changes and additions to BMDP for the new 1990 release. Volume 1 introduces BMDP and describes the most commonly used programs. Volume 2 describes the more specialized programs. Readers can range from new to experienced users. This document supersedes the BMDP Statistical Software Manual (0-520-06473-9).

\section{BMDP USER'S DIGEST (0-520-071158)}

Is a 9-inch by 4-inch, casy-to-carry, greatly condensed version of BMDP Biomedical Computer Programs (the 1979 document). This document places emphasis on the control language and on describing the most efficient methods of using the available program options. This document gives examples for each instruction (parameter) with a short explanation and document reference.

\section{BOEING MATHEMATICAL SUBPROGRAMS LIBRARY}

Is a four-volume set of documents consisting of The Boeing Extended Mathematical Subprogram Library, Computer Supplement for Release 12, Fundamental Subprograms in Release 12, and Advanced Subprograms in Release 12. The set contains information on Release 12 of the BCSLIB Mathematical/Statistical Library provided by Boeing Computer Scrvices. BCSLIB is an American National Standards Institute (ANSI) Fortran 77 subprogram library that provides comprehensive coverage of the basic areas of numerical mathematics and statistics, as well as useful utilitics such as in-core sorting and line printer plotting. The Boeing Extended Mathematical Subprogram Library provides an overview of BCSLIB-EXT and complete usage information for all BCSLIB-EX'T subprograms. Computer Supplement for Release 12 provides information about all system specific features, such as computers and systems for which BCSLIB is available. Fundamental Programs in Release 12 provides an overview of BCSLIB and complete usage information for all fundamental BCSLIB subprograms. Advanced Subprograms in Release 12 provides complete usage information for the advanced programs. Readers should have a working knowledge of Fortran 77.

\section{COMPILER USER'S GUIDE FOR VM/CMS (SC09-1130-01)}

Is a reference guide for programmers writing $\mathrm{C}$ programs under CMS on the System $/ 370$ architecture. It provides an overview of how the compiler works and explains how to compile, link, execute, and debug programs using the System/370 C Compiler on CMS. Readers should be familiar with CMS.

\section{CA-CUECHART USER GUIDE, RELEASE 2.1 (RG99CC2001S)}

Provides information for all levels of users on CACuechart--software for chart making. It has five chapters: "Overview," "Before Getting Started," "Running CA-Cuechart," "Sample Session," and "Sample Charts." The appendixes contain set-up stencils, instruction strings, and data cntry methods. 


\section{CA-DATA CONNECTION USER MANUAL, RELEASE 1.0 (RG99DC1001S)}

Describes THE DATA CONNECTION, an integrated portfolio of tools designed to solve data access problems. As the name suggests, THE DATA CONNECTION builds connecting links between Tellagraf and the many sources of data used to produce meaningful, up-to-date graphical presentations. THE DATA CONNECTION consists of four tools, each of which solves a particular set of data acquisition, data analysis, or data management problems: FILE CONNECTION, REPORT CONNECTION, EXTERNAL PROGRAM CONNECTION, and DECISION SUPPORT CONNECTION. The reader should be familiar with the Tellagraf product, including basic Tellagraf commands and files. This document supersedes the Data Conniction User's Guide.

\section{CA-DISSPLA CODEBOOK: GUIDE TO MAKING CA-DISSPLA CODEBOOK MASTERS (QG 99 CB 10MMS)}

Describes the Codebook interactive option of Disspla and the different Codebook files and subfiles; explains the individual processor commands and their parameters; and shows how to use processor commands together to get input, loop, test conditions (including new subfiles), and direct output. Readers should have a working knowledge of Disspla.

\section{CA-DISSPLA CODEBOOK USER GUIDE (RG 99 CB 1001S)}

Describes the Codebook option of Disspla, its features and uses, and discusses Codebook terminology. This document is for both novice and experienced Disspla users.

\section{CA-DISSPLA FIRST FACTS, VERSION 11.0 (QG 99 DS 11FFS)}

Introduces novice users to the features of the CADisspla graphics system. This document presents a varicty of examples to show you how to generate graphics. Each example is a working Fortran prograrn that produces the graphic show with the code. With CA-Disspla, you cas produce line, bar, and pie charis; Gantl charts and calendars; three-dimensional models of objects; multiple grap.'s or pictures displayed with text; contour diagrams and iartographic mapping; and displays that interactive menus or real-time data acquisition can modify.

\section{CA-DISSPLA POCKET GUIDE, VERSION 11.0 (QG 99 DS 11P1S)}

Is a compact reference that describes technical features and lists rouline parameters for the Disspla Graphics Software package. It also includes several devicedependent routines as well as DISSPOP postprocessor commands and directives. The organization of the Guide is identical to the CA.DISSPLA User Manual. This document supersedes the CA-DISSPLA Pocket Guide, Version 10.5 .

\section{CA-DISSPLA USER MANUAL, VERSION 11.() (RG 99 DS 1101S)}

Consists of two volumes that describe all features coming with the base CA-Disspla package. These features include subroutines that draw axes, curves, simple figures, text, three-dimensional axes, and many tools such as interpolation and blanking.

Volume 1: The Basic Graphics System is divided into ten chapters. Chapter 1 provides a tutorial illustrating how you can create a graphic, explains important CA. Disspla concepts, conventional, and Fortran 66 and 77 concens. Chapter 2 describes how to set up the plot page and establish certain global parameters. Chapter 3 explains how to define and modify axis systems. Chapter 5 includes simple subroutines that draw straight lines and conic sections unrelated to physical data. It also describes headings, annotation, legends, character style, alphabets, line spacing and other text features. Chapter 7 provides subroutines that shade specific or arbitrary areas and let you create continuous tone images from pixel data. Chapter 8 explains the CA-Disspla blanking system and provides sevcral subroutines to use in addition to the automatic blanking subroutines. Chapter 9 describes three-dimensional graphics, including threc-dimensional axis sctup, vicwing, and three-dimensional curve-drawing. Chapter 10 describes subroutines that can act on any CA-Disspla feature.

Volume 2: Options describes cach of the options to CA-Disspla that your site may choose to license. Options is divided into four main sections. The "Presentation Option" section describes the Business Features, Page Layout, and Shaded Fonts features. The "Productivity Option" section describes the Codebook, Mctafiles, and Dynamics features. The "Engincering 
Option" section describes the Mapping, Contouring, Object Rendering, and CA.GKS features. The "Devices" section provides general information for producing graphics on output devices. Readors should have a working knowledge of Fortran,

\section{CA-GRAPHICS CONNEC'TION USER GUIDE, VERSION 1.0 (RG 04 GC 10UGS)}

Describes the CA-Graphics Connection mainframe system avallablo for CMS users.

\section{CAL ASSEMBLER REFERENCE MANUAL, VERSION 2 (SR-2003)}

Is for experienced programmers. The Cray Assembler Language (CAL) Assembler Vorsion 2 allows you to express symbolically all hardware functions of a mainframe for a Cray-2, Cray X-MP, or Cray-1 computer system. This detailed and precise level of programming is especially helpful for tailoring programs to the architecture of a Cray mainframe and for writing programs requiring code optimized to the hardware.

\section{CA-TELLAGRAF BRIEF: BULLETIN OF RELEASE INFORMATION ON ENHANCEMENT FIXES, VERSION 6.1 (UD 99 TG 610BS)}

Describes the new fealures that have been added and the software difficultics that have been corrected in Tellagraf 6.1 and 6.(). This document updates the $C A$ Tellagraf User's Manual.

\section{CA-TELLAGRAF CGM B.R.I.E.F. BULLETIN OF RELISASE INTIORMATION ON ENHIANCEMENTS/FIXES, VERSION 6.1 (UD 99 TG 61CGS)}

Describes how to create a Computer Graphics Metafile (CGM) file in Tellagraf, how to send the CGM file to a printer, how to modify the file before printing, and how to retricve a CGM file into Tellagraf.

\section{CA-TELLAGRAF COMMAND REFERENCE MANUAL, RELEASE 7.0 (R199TG7001S)}

Is a twe-volume document that is a complete reference to using CA-Tellagraf via commands, Volumo $1 \mathrm{con}$. tains descriptions of the data-entry, chart-type, and annotation commands-all the commands you need for day-10-day use. Volume 2 contains information you need less often (such as descriptions of files and operating commands, chart-layout commands, instructionstring commands, and format commands).

\section{CA-TELlAGRAF MENUS PRIMER, RELEASE 1.5 (QG 99 TG 15MPS)}

Provides an introduction to CA-Tellagraf Menus and defines many of the graphics terms and concepts that are used in both CA-Tellagraf Menus and CATellagraf.

\section{CA-TELLAGRAF PRIMER (QG 99 T' 10PRS)}

Provides the reader with a basic working knowledge of Tellagraf and is current with Version 6.1. Chapter 1 describes how to use Tellagraf's basic charting commands to make line, bar, pie, and word charts and publication-quality tables with a step-by-step example of each chart type. Chapter 2 explains how to use the customizing commands in the CA-TELLAGRAF User's Manual and the Pocket Guide. The glossary includes many of the new words that you will learn in the Prim. er lessous, and the appendix is a compendium of customized charts and the commands that produced them. For in-depth information, you should also refer to the CA-TELLAGRAF User's Manual. This document supersedes Tellagraf First Facts.

\section{CA.TWLLAGRAF USER'S MANUAL, VERSION 6.1 (RG 99 'TG 6101S)}

Is a "how to draw" book. Illustrations, cxamples, and sample programs show the reader the benefits of computer graphics, and more specifically, the simplicity of using Tellagraf. Tellagraf is a versatile, flexible, and conversational computer graphics system that produces publication-quality charts and graphs. This document presents the capabilities of Tellagraf in detail: Part A presents a general overview of the system, and the remainder of the document is a reference book that presents each of the Tellagraf commands and options in detail. This document supersedes the Tellagraf User's Manual. 


\section{CENTER FOR ENERGY RESEARCH COMPUTATION PROJECT REPORT FOR FY1988 (ANL/TM 465)}

Contains the findings of the Conter for Encrgy Rescarch Compulation (CERC) Project's first-year efforts.

\section{CENTRAL IBM SYSTEMS AT ARGONNE NATIONAL LABORATORY}

Is a brochure that describes the central IBM systoms avallable at Argonnc. This brochure supersedes the December 1990 brochure.

\section{CENTRAL VAX COMPU'TING AT ARGONNE NA'TIONAL LABORATORY}

Is a brochure that describes the central VAX cluster at Argonne. This brochure supersedes the October 1990 brochurc.

THE CERC REPORT: RESULTS OF THE CERC PROJIECT WITH RECOMMENDATIONS FOR ANL INSTITUTIONAL STRATEGIES IN ADVANCED SCIENTIFIC COMPUTING (ANL/TM 475)

Describes the technical investigations and results obtained during the Center for Energy Research Computation (CERC) Project at Argonne and contains color visualizations of major CERC projects. This document also uses the insights gained through this project into the future of advanced scientific computing at Argonne and other national laboratories to identify strategies that will help to position Argonnc as a leading center of computational science and advanced computing.

\section{CF77 COMPILING SYSTEM, VOLUME 1: FOR'TRAN REFERENCE MANUAL (SR-3071 5,0)}

Is a reference manual for the Fortran compiler that is part of the CF77 compiling system. The compiling system operates on all Cray Research computers and operating systems. This document supersedes the CF77 Compiling System, Volume 1: Fortran Reference Manual (SR-3071 4.()),

\section{CF77 COMPILING SYSTEM, VOLUME 2: COMPILER MESSAGE MANUAL (SR-3072 5.0)}

Lists all messages issued by the compiling phase of the CF77 compiling system. Each message is followed by an expanded description of the problem and possible solutions. This document supersedes the CF77 Compilling System, Volume 2: Compller Message Mianual (SR-3072 4.0)

\section{CF77 COMPILING SYSTEM, VOLUME 3: VECTORIZA'T'ION GUIDE (SG.3073 5.0)}

Explains the use of vector processing with the $\mathrm{CF} 77$ compiling system. The compiling system operates on all Cray Rescarch computers and operating systems.

\section{CF77 COMPILING SYSTEM, VOLUME 4: PARALLEL PROCESSING GUIDE (SG-3074 5.0)}

Describes the CF77 compiling system. This document defines and describes the Autotasking feature of the CF77 compiling system. Autotasking is the automatic distribution of loop iterations to multiple processors. This document supersedes the CF77 Compiling System. Volume 4: Parallel Processing Guide (SG-3074 4.0).

\section{CHANGES AND ENHANCEMENTS TO BASE SAS SOFTWARE FOR PERSONAL COMPUTERS, RELEASE 6.03, SAS TECHNICAL REPORT P-171 (1.55544^071-1}

Describes the changes and enhanecments in base SAS software for Release 6.03. Significant changes and enhancements encompass seven major areas: the SAS Display Manager System (a full screen editor with three new windows and cut-and-paste features); Catalog Conversion (users must convert catalogs created under Release 6.01 to 6.(1) format); Compatibility Features (PRINTTO, DO OVER, and INPUT statements allow users software compatibility with carlier versions of SAS); General Processing (includes greater flexibility in routing output, crror handling, and expanded memory services); Changes in the DATA Step (new features include arrays, delimiters, ten new functions for financial statistics, etc.); Changes to DATA and PROC Step Features (macro features have been greally enhanced); and New Procedures (includes CATALOG, COMPARE, PRINTTO, ctc.), 


\section{CHOOSING 'THE: RIGHT CHAR'T}

Helps you avoid poorly designed computer graphlcs. Whillo computor graphics hardwaro and softwaro aro now becoming widely avallable in business and govcrnmont, many computer-genorated graphics fail to Illustrate graphically or communkate clearly. Such poor design results directly from the fact that programmers, rescarchers, managers or clorical staff, with little or no formal training in graphic design, proparo many computer graphics. When faced with a request to "make a chart," they olten use the "default" styles and designs available from computor graphics packages. Programmers with little experience in graphics design have doveloped inany of these "defaults." This document will make you mors aware of appropriate charts and styles for maximum communication.

\section{CLASS NOTIES HOR A PL/I COURSE}

(ANL-76-70)

Consists of notes for a course in Programming Language/I (PL/I). They might serve as a guide to othcrs who are doveloping a course, and indecd as class notes for that course. They might be useful as a textbook independent of any coursc; as such a textbook, however, they are not self-contained because of the built-in assumption that they will supplement lectures and accompany documents. Very nearly the full langatage is taught here, with the emphasis on concepts rather than practical details. The unorthodox order in which concepts are introduced is the deliberate invention of the author. One effect of this organization is the complete avoidance of any discussion of input/output $(\mathrm{I} / \mathrm{O})$ until roughly the midpoint of the course. These notes should provide students with an enhaneed perception and understanding of the many concepts and their logical relationships.

\section{CMS AT' ANL. (ANL/TM 423, REVISION 2)}

Is an ANL supplement to IBM Virtual Machine/System Product (VM/SP) lechnical documentation on VM/SP. In most instances it serves as the single repository for VM/SP policy and enhancements at Argonne National Laboratory. When appropriate, it points to material available in other documents; it does not ordinarily duplicate information available in vendor documentation or in other CTD technical memoranda. This document supersecies CMS at ANL (ANL/TM 423, REVISION 1).

\section{COMPUTATIONAL SCIENCE AND ENGINEERING AT ARGONNE NATTIONAL II, AYSURAT'TORY}

Is a brochure that describes a few of the ways in which Argonne computational research is opening up new vistas of our physical world.

\section{THE COMPUTING AND TELECOMMUNICATIONS DIVISION SOF'TWARE LIBRARY (ANL/TM 463)}

Explains the policies and procedures of the CTD Software Library. These policies and procedures are necessary to preserve the software documentation and the Laboratory's investment in software. This technical memorandum serves as a practical guide for managers, supervisors, programmers, analysts, and other data processing personnel who need to consult the documentation or use the software available in the Software Library.

\section{CONTINUOUS SYSTEM MODELING PROGRAM III AND GRAPHIC FEATURE GENERAL INFORMATION (GH19.7000)}

Describes the Continuous Simulation Modeling Program (CSMP) III, an IBM program product that aids development and execution of simulation models for continuously changing systems represented by differential equations. CSMP III is a significant extension of the previously available System/360 CSMP. It offers a model-building language based on and including the widely accepted Fortran IV language, a broad selection of formats for displaying or tabulating, simulation results, a symbolic library system for storing and retricving models and commonly used routines, and an optional graphic feature that dramaticaliy reduces model development time by providing interaction between man and machine at all stages of the simulation process.

\section{CONTINUOUS SYSTEM MODELING PROGRAM III (CSMP III) OPERATIONS GUIDE} (SH19-7002)

Contains information and procedures that enable the reader to retrieve machine-readable material from the basic distribution tape, to create the required system load modules, to incorporate frequently used procedures into the operating system (OS) procedure library, and to execute the supplied sample model. It also 
describes how to modify the CSMP III source and object modules to build the modified system, to back up such a modifiod system, and to maintain the CSMP III Symbolic Library datasct.

\section{CRAY C REFERENCE MANUAL (SR-2024D)}

Summarizes the characteristics of the $\mathrm{C}$ language and describes the differences between the Cray $\mathrm{C}$ implementation on the various Cray computer systems. Readers should have a working knowledge of the $\mathrm{C}$ programming language and the Unix-Based Cray Operating Systom (UNICOS), This document supersedes the Cray C Reference Manual (SR-2024).

\section{CRAY COMPUTING AT ARGONNE NATIONAL LABORATORY}

Is a brochure that describes the Unix-Based Cray Operating System (UNICOS) environment at Argonne. This brochure supersedes the October 1990 brochure.

\section{CRAY RESEARCH, INC. SILVER BINDER} (1 INCH)

Is a 1-inch silver binder for Cray documentation; it contains no written material.

\section{CRAY RESEARCH, INC. SILVER BINDER} (1.5 INCH)

Is a 1.5 -inch silver binder for Cray documentation; it contains no written material.

\section{CRAY RESEARCH, INC. SILVER BINDER (2 INCH)}

Is a 2-inch silver binder for Cray documentation; it contains no written material.

\section{CRAY RESEARCH, INC. SILVER BINDER (3 INCH)}

Is a 3-inch silver binder for Cray documentation; it contains no written matcrial.

\section{CRAY RESEARCH, INC. SILVER BINDER PRIMER (1.5 INCH)}

Is a 1.5 -inch silver binder for the Cray primer; it contains no written material.

\section{CRAY STANDARD C PROGRAMMER'S REFERENCE MANUAL (SR-2074 3.0)}

Describes the Cray Standard C features and summarizes Cray-specific details of the American National Standards Institute (ANSI) standard C language. Readers should have a working knowledge of the $\mathrm{C}$ programming language and the Unix-Based Cray Operating System (UNICOS). This document supersedes the Cray Standard C Programmer's Reference Manual (SR-2074).

\section{CRAY Y-MP, CRAY X-MP, AND CRAY-1 C LIBRARY REFERENCE MANUAL (SR-(1)136C)}

Describes the $C$ library routines available with the Cray Operating System (COS) and the Unix-Based Cray Operating System (UNICOS), running on a Cray X-MP or Cray- 1 computer system. This document is a reference manual for $\operatorname{COS}$ and UNICOS programmers. It assumes the reader has a working knowledge of COS, UNICOS, or the Unix operating system.

\section{CTD INFORMATION PACKET}

Describes the mission, equipment and services, availability, history, and organization of CTD history at Argonne National Laboratory.

\section{CTD SOFTWARE LIBRARY MAIN'TENANCE TOOLS FOR MIS SOFTWARE (ANL/TM 468)}

Describes ANL computing policy for ensuring procedural consistency and systems protection on the design, writing, and modification of program master libraries by the CTD Software Librarian or by Management Information Systems (MIS) analysts. This document supersedes Computing Services Software Library Program Maintenance Tools for Information Systems Sec-tion Software (ANL/TM 443). 


\section{CTD WRITING AND EDITING STANDARDS (ANL/TM 487)}

Provides information that will help writers produce better documents consistent with CTD standards in less time. This document offers good advice for CTD writers, specifies approved generic Script files for formatting documents in standard departmental formats, establishes the publication processes for CTD publications, and provides explanations and examples of special usage conventions. This document supersedes Computing Services Writing and Editing Standards (ANL/TM 429, REVISION i).

\section{CUECHART S TENCIL MAKER'S GUIDE}

Helps the Tellagraf user modify the Cuechart standard stencils and prepare new custom stencils. The "stencil" contains both Tellagraf commands to produce a basic chart and Cuechart commands that contain prompting information. The "stencil" is the key to the flexibility and expandability of the Cuechart system. It is called the "Cuechart" when the user looks through the Cuechart User's Guide to select a particular chart style and to determine the CHART-ID.

\section{DATA PHYSICIAN PLUS? COMPUTER VIRUS PROTECTION SYSTEM}

Is a document and a diskette with two kinds of virus scanning programs: RESSCAN (a memory-resident program to scan for viruses) and VirHUNT (a diskresident program to identiî/remove viruses). You can use these programs with IBM PCs; IBM XTs; IBM ATs; or compatibles having a flexible disk drive, $256 \mathrm{~K}$ available RAM, and DOS 2.0 or higher.

\section{DESIGN IDEAS USING TABLES}

Assumes that the reader already knows how to use Tellagraf to create computer graphics. Published by the Integrated Software Systems Corporation (ISSCO), this document explains how to use the Tables language to produce a new type of chart (the table) with considerable flexibility (e.g, with such features as columns of numbers, bars, annotations, and interstitial lines). Tables can accommodate the traditional types of tables, but it also allows for considerable creativity. Most users will want to use this document as a reference guide by looking at the many exampies and aủaptining selected specifications for inclusion in their own tables.

\section{DFSORT APPLICATION PROGRAMMING GUIDE, RELEASE 11 (SC33-4035-14)}

Is for programmers who wish to sort or merge files with the DFSORT (Data Facility Sort) Program Product. To use this document, you should have a basic understanding of OS/VS and its jub control language (JCL). Using this document, you will be able to prepare all the input necessary 10 perform a sont or merge. You will also be able to link your own routines to DFSORT to perform such services as altering, deleting, or inserting records as they are being sorted or merged.

\section{DISASTER RECOVERY PLAN FOR ADMINISTRATIVE SYSTEMS (ANL/TM 456, REVISION 1)}

Is Volume I of three volumes. Only this volume is printed as a technical memorandum. Volume I describes the organization and responsibilities of the recovery teams and includes procedures for recovery in the event of a disaster. The three volumes of the Plan represent a fully workable disaster recovery plan for service disruptions that last more than three days. This document supersedes the Disaster Recovery Plan for Administrative Systems (ANL/TM 456).

\section{DISINFECTANT V2.8}

Is a $31 / 2$-inch diskette for the Apple Macintosh computers that can detect and cure the following viruses: Scores, nVIR, INIT 29, ANTI, MacMag, WDEF, ZUC, MDEF, Frankic, CDEF, MBDF, INIT 1984, and CODE 252.

\section{DISTRIBUTION OF MATHEMATICAL SOFTWARE VIA ELECTRONIIC MAIL (ANL/MCS-TM-48)}

Explains how to use the netlib server-a Unix network service that makes a large collection of public domain mathematical software available through electronic mail.

\section{DM USER'S GUIDE}

Describes how to use the Directory Management Utility to manage, clean up, and work with your files and dirceitory strictiurc. 


\section{DO LOOP TRANSFORMING TOOLS}

Is an overview of three transformation tools contributed to the Toolpack/1 tool collection. The first tool unrolls out DO loops, the second condenses sequences of inner DOs in unrolled outer DOs into single DO loops together with assignment statements, and the third combines sequences of assignment statements resulting from the action of the first two tools. Acting in concert with one another and with other Toolpack/1 tools, these tools transform ccrtain classes of Fortran DO nests that typically occur in linear algebra software. The effect is to improve the performance of such software on vector machines by reducing the number of words transferred between memory and the vector registers. The paper emphasizes features of the Toolpack tool-writing environment that are employed in writing such transformation tools. This paper is available through the Mathematics and Computer Science Division (extension 2-7222).

\section{ELECTRONIC MAIL AT ANL (ANL/TM 431, REVISION 2)}

Discusses (1) concepts, issues, and commands basic to electronic mail and (2) capabilitics and limitations of elcctronic mail systems at ANL, with emphasis on communication through BITnet. This document contains five chapters and seventeen appendixes. Chapter 1 explains how electronic mail may benefit you and answers many basic questions about this communications system. It defines electronic mail, compares it with conventional mail and telephone communication, lists the benefits electronic mail users enjoy, and familiarizes readers with the electronic mail universe accessible to Argonne. Chapter 2 defines three electronic mail concepts: (1) addresses, (2) networks, and (3) gateways. It also explains how to find electronic mail addresses, how to communicate electronically when at home or travelling, and how systems format electronic mail files. Chapter 3 explains how users send electronic mail through the electronic mail systems at Argonne. Structured descriptions of each mail system provide answers to basic questions on how to use each systein. Chapter 4 describes the three major Laboratory-wide electronic mail networks at Argonne-Network Job Entry (NJE), the Digital Equipment Corporation network (DECnet), and the Transmission Control Protocol/Internet Protocol (TCP/IP)--and their connections. Chapter 5 describes which networks and network gateways are available to Argonne electronic mail users. This chapter provides such information as what the networks are, their size, and where they are located. The appendixes provide more details about the different networks. This document supersedes Electronic Mail at ANL (ANL/TM 431, REVISION 1).

\section{EXPERT SYSTEMS TOOLS AND APPLICATIONS (0-471-83950-7)}

Is about expert systems and how they are changing the business world. The term expert systems describes a whole set of concepts, procedures, and techniques that enable business people to use computers in a varicty of valuable ways. In essence, expert systems techniques enable computers to assist people in analyzing and solving complex problems that can be stated only in verbal terms. This document has four sections. The first section provides an overview of the expert systems market as it stood at the beginning of 1987. The second section discusses in detail several expert system building tools. The third section presents an overview of the expert systems development process. The fourth section provides a catalog that describes most of the fielded expert systems applications (at the beginning of 1987). This document focuses on the immediate commercial benefits to be derived from the existing generation of expert systems.

\section{FORMS DESIGN REFERENCE GUIDE FOR THE IBM 3800 PRINTING SUBSYSTEM (GA26-1633)}

Provides necessary information for those responsible for the design of forms and the preparation of overlay negatives used by the IBM 3800 Printing Subsystem.

\section{FORTRAN (CFT) REFERENCE MANUAL (SR-0009-M)}

Is for Cray Fortran programmers who have a working knowledge of the Fortran programming language. The Cray Fortran Compiler translates Fortran language statements into Cray Assembly Language programs that make effective use of the Cray X-MP and Cray-1 computer systems. The document explains the Cray Fortran language in its entirety and describes the related characteristics of the Cray Operating System (COS) and the Unix-Based Cray Operating System (UNICOS) where applicable. 
FORTRAN 90 EXPLAINED (0-19-853772-7)

Is a complete reference work. The first chapter sets out the background to the work on the new standard. The following nine chapters describe Fortran 90 less its redundant and obsolescent features. Some knowledge of programming concepts, although not necessarily of Fortran 77, is assumed.

\section{FUNPACK--A PACKAGE OF SPECIAL FUNCTION SUBROUTINES (TM 385)}

Describes FUNPACK, a modest systematized collection of machine specific special function Fortran programs prepared under the NATS (National Activity to Test Software) project. Versions exist only for largescale IBM, Control Data Corporation (CDC), and Univac computing systems. The package includes subroutines to evaluate certain Bessel functions, complete elliptic integrals, exponential integrals, Dawson's integral, and the psi function with secondary entries for exponential scaling or alternative arguments where appropriate. The package also includes a versatile error-monitoring routine.

\section{GETTING STARTED WITH FORTRAN 77}

Describes the Fortran 77 computer language. Fortran 77 is the current Fortran standard used by all computer vendors. This document is for a beginning student.

\section{GNU EMACS MANUAL}

Documents the use and simple customizing of the Emacs cditor, Version 18. This document is primarily a reference manual, but it can also be used as a primer.

\section{GNU EMACS REFERENCE CARD}

Summarizes the commands for the Emacs editor, Version 18.

\section{GRAPHICS TOOLS FOR DEVELOPING HIGH-PERFORMANCE ALGORITHMS (MCS-P28-1288)}

Discusses two tools that aid in the development of parallel algorithms for parallel computers. The tools are SCHEDULE. which provides an environment for developing and analyzing new parallel algorithms in
Fortran, and a graphics tool for studying memory access patterns of algorithms. This paper is available through the Mathematics and Computer Science Division (extension 2-7222).

\section{GUIDE TO COMPUTER PROTECTION AT ANL (ANL/TM 413)}

Is for those persons who are responsible for certain kinds of sensitive applications, sensitive data, or a computer that processes sensitive applications or sensitive data. To determine whether the applications, data, or computer for which you are responsible is sensitive, contact the appropriate Computer Protection Representative who coordinates the sensitive applications and computer protection for your division, program, or department. The parts of this document that you find relevant will depend on your responsibilities to protect unclassified but sensitive information. (Note that this document does not cover the procedures for protecting classified information.) Chapter 2 summarizes the responsibilities for administering Argonne National Laboratory's policy on the protection of computerized unclassified sensitive information and suggests a plan for executing the policy. Chapter 3 describes the management control process for verifying compliance with the Argonne policy. Chapters 4 through 7 define the major areas addressed by the policy and gives you guidelines for complying with the policy in these major areas.

\section{GUIDE TO COMPUTING AT ANI. (ANL/TM 336, REVISION 2)}

Provides a general overview of the central computing facilities for those interested in using those computers at Argonne National Laboratory. This information will assist you in selecting the computer, system, network, and software with which you can do your work most efficiently. This document supersedes Guide to Computing at ANL (ANL/TM 336, REVISION 1).

\section{GUIDE TO REPORTING TIME IN THE FINANCIAL INFORMATION SYSTEM AT ANL (ANL/TM 414)}

Describes the operation of a new system with which the full cost recovery service centers of the Laboratory report employee time, whether the time is charged to another organization or to an internal function. Most service centers of the Laboratory currently report 
employee time to the Financial Information System (FIS) via time cards or time sheets, which are routed to the Data Entry group of the Administrative Data Processing Operations, where the time records are keyed. With this new system, the service center assumes primary responsibility for the keying and management of time charges. The system provides these benefits to the service center: a choice of period and month-end boundary, printed time sheets with history of charges (thus fewer rejected charges), and computer-readable files of current and prior charges for analysis.

\section{GUIDE TO TELECOMMUNICATIONS AT ANL (ANL/TM 422)}

Provides an overvicw of ANL's telecommunication resources and services. These telecommunication resources and scrvices connect ANL-East and ANLWest with universitics, other national laboratories, and government departments and agencies in the United States and abroad. These resources and services encompass voice, data, radio, pubiic address, and alarm systems. This document also covers CTD procedures that are part of the decision-making process involved in fulfilling communications requirements. This document is the primary source of information on ANL telecommunications.

\section{GUIDE TO USING VMS COMMAND PROCEDURES (AA-LA11A-TE)}

Presents key concepts and techniques for developing command procedures with the Virtual Address Extension/Virtual Memory System (VAX/VMS) Digital Command Language (DCL). Many examples, including examples of complete command procedures, demonstrate applications of the concepts and techniques discussed. The examples include elementary, advanced, and complex command procedures. This document supersedes Guide to Using DCL and Command Procedures on VAX/VMS (AA-Y501A-TE).

\section{GUIDE TO VAX/VMS NJE NETWORKING AT ANL (ANL/TM 444)}

Describes how to implement, operate, and use the Network Job Entry (NJE) emulator. The NJE emulator consists of locally written software that allows Digital Equipment Corporation (DEC) VAX computers using the Virtual Memory System (VMS) to participate in an IBM NJE network. The NJE emulator permits VAX/VMS users to interface with the $\overline{\mathrm{IB}} \overline{\mathrm{M}}$
Conversational Monitor System (CMS) and Wylbur users and to submit jobs to the Multiple Virtual Storage (MVS) batch system at CTD. This document supersedes the Friendly Neighborhood Computer Project: Extension of the IBM NJE Network to DEC VAX Computers (ANL/TM 383).

\section{GUIDE TO VMS FILES AND DEVICES (AA-LA06A-TE)}

Describes some of the routine tasks that general users perform on files and devices. This document is for all general users.

\section{A GUIDEBOOK TO FORTRAN ON SUPERCOMPUTERS (0-12-444760-0)}

Explains in detail both the underlying architecture of today's supercomputers and the manner by which a compiler maps Fortran code onto that architecture. Most important, the constructs preventing full optimizations are outlined, and specific strategies for restructuring a program are provided. It is assumed that the reader has a working knowledge of Fortran and that the reader's principal programming experience has been on the classical "von Neumann" machines: sequential, scalar processors.

\section{GUIDELINES FOR DOCUMENT DESIGNERS (400-78-0043)}

Complements CTD Writing and Editing Standards (ANL/TM 487). This desktop reference guide provides guidelines and principles for making public documents easier to read and understand. The National Institule of Education produced this document specifically for professionals who must write a great deal in their jobs but are not trained writers.

\section{GUIDELINES FOR USING THE AMDLIB, IMSL, AND NAG MATHEMATICAL SOFTWARE LIBRARIES AT ANL (ANL-81-73)}

Summarizes the numerical software subroutines in the Applied Mathematics Division Subroutine Library (AMDLIB), the International Mathematical and Statistical Libraries, Inc. (IMSL), and the Numerica Algorithms Group, Ltd. (NAG) mathematical librarics. This document discusses 17 numerical analysis subjects and lists the appropriate subroutines available in the threc 
libraries for solving cach type of problem, with our recommendations for particular types of applications. The appendixes discuss the organizations of the three libraries and the methods available for accessing particular subroutines in each library.

\section{HANDBOOK OF TECHNICAL WRITING, THIRD EDITION (0-312-35810-5)}

Contains much new and updated information on English grammar, usage, style, format, and writing procedures (planning, rescarch, outlining, and methods of development). C'TD Writing and Editing Standards (ANL/TM 487) recommends this book as a complementary reference guide. This book provides information on and examples of various kinds of technical communication--reports, proposals, instructions, specifications, job descriptions, letters, and memoranda. It is especially helpful as a desktop reference because it offers four mans of access to information. (1) It is organized alphabetically so that you can go immediately to the topic you need. (2) It contains an index that includes not only terms actually used in the alphabetical entrics but also common synonyms for those terms. (3) It contains a "Topical Key to the Alphabetical Entrics" that groups entrics into more general catcgorics so that you can more casily sce relationships among entries. (4) It offers "Five Steps to Successful Writing," a brief review of the writing process that includes page references to pertinent entries.

\section{HIGHLIGHTS OF SPEAKEASY EPSILON}

Describes Speakcasy IV Epsilon, which is an enhanced version of the 1985 Delta relcasc of Spcakcasy IV. This document includes changes and new features in Spcakcasy IV Deltat and supplements the Speakeasy IV Reference Manual.

\section{IISTORIAN PLUS USER'S MANUAL}

Describes how to use the Historian Plus Software Control System. This system is a software package that cnables you to storc and update an entire collection of source nuatcrials (called a library) while it keeps track of all changes you make to that library. The system executes in batch or interactive mode and provides a means for transporting librarics across machine bound. arics. Anyone who has a computerized collection of information that necds to be changed from time to time can profitably use Historian Plus and this document.

\section{IBM BASIC APPLICATION PROGRAMMING: SYSTEM SER VICES (SC26-4028)}

Is for Basic application programmers who need IBM Basic specific guide and reference material to run IBM Basic programs under VM/SP CMS. The first chapter gives guidance information on how to use IBM Basic effectively in CMS, with explanations and examples of CMS commands useful with IBM Basic. The second chapter gives reference information; it lists $\mathrm{CP}$ and CMS commands used most often by the Basic programmer. This document discusses options relevant to Basic.

\section{IBM BASIC LANGUAGE REFERENCE (GC26-4026-2)}

Provides reference material on the IBM Basic language. It presents definitions and examples of IBM Basic statements and commands. Readers should have some knowledge of the Basic language. This document supersedes the IBM BASIC Language Reference (GC26-4026).

\section{IBM BASIC PROGRAMMING GUIDE (SC26-4027-2)}

Describes how to use IBM Basic to design, develop, test, and run programs in the Basic language. It also provides guidance for using IBM Basic to develop Basic programs. Readers should have some knowledge of the Basic language. This document supersedes the IBM BASIC Programming Guide (SC26-4027).

\section{IBM BINDER FOR PROFS DOCUMENTS (SB30-0480)}

Is a binder for IBM Professional Office System (PROFS) documentation; it contains no written material.

\section{IBM C LANGUAGE MANUAL (SC09-1128-01)}

Describes the $\mathrm{C}$ programming language and associated C library for the System $/ 370$ architecture running CMS, MVS, or MVS/XA. It serves as a reference guide for programmers writing programs in the $C$ language. Readers should have a working knowledge of programming fundamentals. 


\section{IBM INTERACTIVE SYSTEM PRODUCTIVITYY FACILITY/PROGRAM DEVELOPMENT FACILITY VERSION 2 RELEASE 3 FOR MVS EDIT AND EDIT MACROS (SC34-4121-00)}

Describes (1) how to use the Program Development Facility (PDF), (2) how to write edit macros, (3) sample CLIST and program edit macros, (4) error handling and edit macro testing, and (5) the edit line, primary, and macro commands available for the Interactive System Productivity Facility (ISPF)/PDF. This document is for application and system programmers who develop and/or use the PDF editor and edit macro instructions. Users should be familiar with coding CLISTs or programs in the MVS environment.

\section{IBM INTERACTIVE SYSTEM PRODUCTIVITY FACILITY/PROGRAM DEVELOPMENT FACILITY VERSION 2 RELEASE 3 FOR MVS GUIDE (SC34-4118-00)}

Provides information about using PDF, including (1) introductory information, (2) library and datasct information, (3) using the PDF options, (4) understanding the outpul listing formats, (5) examples of character translation tables, (6) lists of abbreviations, (7) descriptions of allocation datasets, and (8) descriptions of the SuperC program. This document is for application programmers using the ISPF/PDF.

\section{IBM OS FORTRAN IV (H EXTENDED) COMPILER PROGRAMMER'S GUIDE} (SC28-6852)

Describes the steps to compile, linkedit, and execute a Fortran IV program using the Fortran IV (H Extended) compiler, an IBM Program Product that operates under the control of the operating system. This document details the methods of invoking each step, input to the steps, and output from the steps. In addition, this document discusses compiler options, features of the operating system used by the Fortran programmer, and practices for coding more efficient Fortran programs. This document is for programmers familiar with the Fortran IV language. Previous knowledge of the operating system is not necessary. Information in this publication about OS/VS2 is for planning purposes until that product is available.

\section{IBM OS/VS COBOL COMPILER AND LIBRARY PROGRAMMER'S GUIDE (SC28-6483-2)}

Provides the necessary information to enable programmers to compile, linkedit, and execute or to compile and load OS/VS Cobol Compiler and Library Programs under the control of the IBM Operating System. The Cobol language is described in IBM VS Cobol for OS/VS (GC26-3857), which is a co-requisite of this document. This document supersedes IBM OS/VS Cobol Compiler and Library Programming (SC28-6483-1).

\section{IBM PROFESSIONAL OFFICE SYSTEM QUICK GUIDE (SX20-2396)}

Applies only to use of PROFS on IBM 3270-type terminals. This document summarizes how to schedule appointments, revicw the in-basket, search and retrieve documents, send a message or note, prepare a document, file documents, alternate the function menu, handle the maillog, and check the mail status. For further details, refer to the PROFS User's Guide.

\section{IBM SYSTEM/370 AND 4300 PROCESSOR BIBLIOGRAPHY (GC20-0001-3)}

Describes technical publications that document machine components, System Control Programming (SCP) and Selectable Units, DB/DC and system sup. port licensed programs, and other supplementary information (e.g., forms and PTF listings). Those who plan for, install, program, or operate an IBM System/370 or 4300 processor should use this bibliography. Features and kinds of information included are titles and abstracts with page counts, clustering and sequencing of items by title, second listing in order-number sequence with subject codes explained and indexed, specific page look-up by program acronym, description of selectable unit publications and their distribution, current status of the publications listed with TNLs, SLSS publication profiles used in distribution with an explanation of these profiles, and details on order numbers (including pscudonumbers). For System/370 publications on industry systems and licensed application programs, refer to IBM System/370 and 4300 Processors: Bibliography of Industry Systems and Applications Programs (GC2()-0370-2). 


\section{IBM SYSTTEM/370 AND 4300 PROCESSORS: BIBLIOGRAPHY OF INDUSTRY SYSTEMS AND APPLICATIONS PROGRAMS $(\mathrm{GC} 20 \cdot-0370 \cdot 2)$}

Describes technical publications needed by those who plan for, install, program, or operate industry systems and licensed application programs for System/370) and 4300) Processors. Industry (sub)systems covered are the IBM 3600 Finance Communication System, the IBM 3730 Distributed Office Communication System, and all others (including the IBM 3790 Communication System). Licensed programs included are all completed applications running under System/370 or 4300 Processor programming (or under Type I programming if for System/370) or 4300 Processors only). Completed applications are those for specific industrics such as finance, insurance, or manufacturing and those applicable to many industrics. Features and the kinds of information included are title and abstracts with page counts, clustering and secquencing of $14 \% 1$ hy title, second listing in order-number sequence $v$ subject codes explained and indexed, specific page look-up by program acronym, latest editions of publications listed with TNLs, SLSS publication profiles used in distribution with an explanation of these profiles, and details on order numbers (including pscudonumbers). For System/37) machine components, support programming, $\mathrm{DB} / \mathrm{DC}$, and supplementary information, refer to the IBM System/370 and 4.300 Processor Bibliography $(\mathrm{GC} 2(0-())() 1-3)$.

\section{IBM SYSTEM/370) EX'TENDED ARCHITECTURE: PRINCIPLES OF OPERATION (SA22-7085-1)}

Provides, for reference purposes, a detailed definition of the machine functions performed by systems operating in the System/37) extended-architecture (370)-XA) mode.

\section{IBM SYSTEM/370 FOR'TRAN H EX'TENDED OPTIMIZATION ENHANCEMENT PROGRAM DESCRIPTION AND OPERA'TOR'S MANUAL} (SH20-2100)

Contains reference information for the IBM System/370 Fortran H Extended Optimization Enhancement. The Optimization Enhancement provides a higher level of optimization for the compilation of Fortran language source programs into exccutable object programs. It also provides faster versions of the most commonly used mathematical subroutines in the
Fortran library and of the interpretation and conversion subroutines necessary for formatted input and output. The Optimization Enhancement requires no changes to Fortran source programs and generally results in object progranis that run faster than those executed under the Standard Fortran $H$ Extended compiler and library. The amount of improvement varies from program to program and from one System/370 model to another. In general, the larger programs on larger systems obtain the best results.

\section{IBM SYE'EM/370: PRINCIPLES OF OPERATION (GA22-7000)}

Provides, for reference purposes, a detalled definition of the machine functions performed by System/370. This document describes each function to the level of detail that must be understood to prepare an assembly language program that relics on that function. It does not, however, describe the notation and conventions that the user should employ in preparing such a program. The user must instead refer to the appropriate assembly language documunt. This document provides information principally for use by assembly language programmers, although anyone concerned with functional details of System/370 will find it useful. Note that this document is a reference document and is not an introduction or a textbook for System/370, It assumes the reader has a basic knowledge of dataprocessing systems, and, specifically, the System/370, which can be obtained from the Introduction to IBM Data Processing Systems (GC20-1684) and the IBM System/370 System Summary (GA22-7001). The information presented in this document is grouped into 14 chapters and several appendixes. The major topics presented include Control, Dynamic Address Translation Interruptions, Multiprocessing, System Control Instruction, General Instructions, Decimal Instructions, Floating Point Instructions, Machine-Check Handling, Input/Output Operations, and System Console.

\section{IBM SYSTEM/370: REFERENCE SUMMARY (GX20-1850)}

Is primarily for use by System/370 assembler language programmers. It contains basic machine information on Models 115 through 168 summarized from the $I B M$ System/370: Principles of Operation (GA22-7000), frequently used information in VS and VM Assembler Language (GC33-4010), command codes for various I/O devices, and a multi-code translation table. 


\section{IBM TRANSMISSION CONTROL PROTOCOL/INTERNET COMMAND RFFERENCE MANUAL (GC09.1204-02)}

Describes the commands used when working with the program called Transmission Control Protocol/Internet Protocol (TCP/IP) for VM. This document assumes the user is familiar with VM, the IBM Control Program (CP), the IBM Conversational Monitor System (CMS), and their commands.

\section{IBM TSO EXTENSIONS VERSION 2 COMMAND REFERENCE (SC28.1881-3)}

Describes the syntax and function of the commands and subcommands of the TSO/E command language and Session Manager, It provides only reference material and assumes you are experienced in the use of TSO/E and Session Manager. The two sections in this document are "TSO/E Commands and Subcommands" and "Session Manager Commands." This document supersedes the OS/VS2 TSO Command Language Reference (GC28-()646-4).

\section{IBM TSO EXTENSIONS VERSION 2 USER'S GUIDE (SC28-1880-2)}

Is a general guide for using Time Sharing Option Extensions (TSO/E). It expands the concepts and basic tasks that are presented in the TSO/E Version 2 Primer. Anyone who uses TSO/E should read this book to learn more about TSO/E in relation to command usage and dataset management. The four sections in this document are "General TSO/E Functions," "Using Data Sets," "Running a Program," and "Changing the Way You Use TSO/E." This document supersedes the OS/VS2 TSO Terminal User's Guide (GC28-0645-4).

\section{IBM VIRTUAL MACHINE FACILITY/370: DIRECTORY MAINTENANCE GUIDE FOR GENERAL USERS (SC20-1839)}

Is for the $V M / 370$ system general user who needs to modify control statements in his own directory entry. The changes that he can affect are restricted to those that do not involve system resources. All other changes must be requested from the System Administrator.

\section{IBM VIRTUAL MACHINE FACILITY/370: GLOSSARY AND MASTER INDEX (GC20-1813)}

Provides guidance to the VM/370 library in threc ways. The glossary defines terms particular to VM/370) that are not included in the IBM Data Processing Glossary (GC20-1699). The master index directs users to the $\mathrm{VM} / 370$ documents that contain the information necded. The command and operand summaries direct users to the publications that document each of the commands and operands available to VM/370 users.

\section{IBM VIRTUAL MACHINE FACILITY/370: IN'TRODUCTION (GC20-1800)}

Introduces $\mathrm{VM} / 370$ and is for anyone who is interested in $\mathrm{VM} / 370$. However, the reader should have a basic understanding of IBM data processing. VM/370 (Virtual Machinc/370) is a System Control Program (SCP) that tailors the resources and capabilities of a single System/370 computer to provide concurrent users their own unique (virtual) machine. VM/3'70 consists of a Control Program (CP), which manages the real computer, a Conversational Monitor System (CMS), which is a general-purpose conversational time-sharing system that executes in a virtual machine, a Remote Spooling Communications Subsystem (RSCS), which spools files to and from geographically remote locations, and an Interictive Problem Control System (IPCS), which provides problem analysis and management facilities. The first section of this document is an introduction; it describes what VM/370 can do. The second, third, and fourth sections describe CP, CMS, and RSCS, respectively. The appendixes include information about system requirements and related VM/370 documents for CMS Users. This document is a prerequisite for the VM/370 system library.

\section{IBM VIRTUAL MACHINE LIBRARY BINDER (SX24-5129)}

Is a binder for IBM documentation; it contains no printed material.

\section{IBM VIRTUAL MACHYNE/EXTENDED ARCHITECTURE SYSTEM PRODUCT VM/XA SP, RELEASE 1 AND RELEASE 2: CMS PRIMER (SC23-0368-0)}

Teaches you how to do your work by using the Virtual Machine/Extended Architecture System Product (VM/XA SP) system and a display terminal. 


\section{IBM VIRTUAL MACHINI/LXTENDED ARCHITEC'TURE SYSTEM PRODUCT VM/XA , SP, RELEASE 1 AND RELEASE 2: CMS PRIMER SUMMARY OF COMMANDS (SC23-0421-0)}

Is a card that summarizes all of the commands presentcd in the IBM Virtual Machine/Extended Architecture System Product VM/XA SP, Release 1 and Release 2: $C M S$ Primer, arranged by chapter and page number.

\section{IBM VIR'TUAL MACHINE/EXTENDED ARCHITECTURE SYSTEM PRODUCT VM/XA SP, RELEASE 1 AND RELEASE 2: CMS USER'S GUIDE (SC23-0356-0)}

Describes the interactive facilities of CMS and VM/XA SP and includes examples showing you how to use CMS and selected functions of VM/XA SP. This document is for the general user of the Conversational Monitor System (CMS) running under the Virtual Machine/Extended Architecture System Product (VM/XA SP).

\section{IBM VIRTUAL MACHINE/EXTENDED ARCHITECTURE SYSTEM PRODUCT VM/XA SP, RELEASE 1 AND RELEASE 2: EXEC 2 REFERENCE (SC23-()361-0)}

Delines the EXEC 2 language. It is to be used primarily as a reference manual; it contains all of the formats, syntax rules, and descriptions of the arguments for EXEC 2 statements.

\section{IBM VIR'TUAL MACHINE/EXTENDED ARCHITECTURE SYSTEM PRODUCT VM/XA SP, RELEASE 1 AND RELEASE 2: SYSTEM PRODUC'T EDITOR COMMAND AND MACRO REFERENCE (SC23-(1)372-0)}

Contains all of the command formats, syntax rules, and operand and option descriptions for the XEDIT command and XEDIT subcommands and macros.

\section{IBM VIRTUAL MACHINE/EXTENDED ARCHITECTURE SYSTEM PRODUCT VM/XA \\ SP, RELEASE 1 AND RELEASE 2: SYSTEM PRODUCT EDITOR USER'S GUIDE (SC23-0373-0)}

Gives you a working knowledge of the System Product editor (also called XEDIT). The first three chapters are for data processing novices. The last four chapters are for new users who have mastered the fundamentals and for data processing professionals.

\section{IBM VIRTUAL MACHINE/EXTENDED ARCHITECTURE SYSTEM PRODUCT VM/XA SP, RELEASE 1 AND RELEASE 2: SYSTEM PRODUC'T IN'TERPRETER REFERENCE} (SC23-0374-0)

Describes the Virtual Machine/Extended Architecture System Product (VM/XA SP) System Product Interpreter and the Restructured EXtended eXecutor (REXX) language. This document includes the use and syntax of the language and explains how the interpreter "interprets" the REXX language as a program is exccuting. This document is for experienced programmers, particularly those who have used another highlevel language (for example, PL/I, Algol, or Pascal).

\section{IBM VIRTUAL MACHINE/EXTENDED ARCHITECTURE SYSTEM PRODUC'T VM/XA SP, RELEASE 1 AND RELEASE 2: SYSTEM PRODUCT INTERPRETER USER'S GUIDE}

(SC23-0375-0)

Describes the programming language called the Restructured EXtended eXecutor (REXX) language. This document also describes how the System Product Interpreter processes or "interprets" the REXX tanguage. This document is for beginners and programmers who have not used a "structured" language before.

\section{IBM VIRTUAL MACHINE/EXTTENDED ARCHITECTURE SYSTEM PRODUCT VM/XA SP, RELEASE 2: CMS COMMAND REFERENCE (SC23-0354-1)}

Describes the command formats, syntax rules, and operand and option descriptions for the Virtual Machine/Extended Architecture System Product (VM/XA SP) CMS commands. This document is for anyone who uses CMS commands. 


\section{IBM VIRTUAL MACHINE/EXTENDED ARCHITECTURE SYSTEM PRODUC'T VM/XA SP, RELEASE 2: CP COMMAND REFERENCE (SC23-0358-1)}

Describes the command formats, syntax rules, operands, and options for the Virtual Machine/Extended Architecture System Product (VM/XA SP) control program (CP) commands. This document is for anyone who uses CP commands.

\section{IBM VIRTUAL MACHINE/EXTENDED ARCHITECTURE SYSTEM PRODUCT VM/XA SP, RELEASE 2: SYSTEM MESSAGES AND CODES REFERENCE (SC23-()376-1)}

Lists and explains the messages and codes that the Virtual Machine/Extended Architecture System Product (VM/XA SP) issues. It also recommends actions a user can take in response to the message or code received. This document is for any user of VM/XA SP who wants information about the codes and messages the system issucs.

\section{IBM VS COBOL FOR OS/VS (GC26-3857-04)}

Describes IBM OS/VS Cobol. It gives the rules for writing Cobol source programs that are to be compiled by the OS/VS Cobol compiler. Its use is as a reference manual in writing OS/VS Cobol programs and as a supplement to the IBM OS/VS Cobol Compiler and Library Programmer's Guide (SC28-6483-2) and the IBM OSIVS Cobol Language Reference Summary (GX26-3720). This document supersedes IBM VS Cobol for OS/VS (GC26-3857-03).

\section{IBM VS FORTRAN VERSION 2 LANGUAGE AND LIBRARY REFERENCF, RELEASE 5 (SC26-4221-6)}

Contains reference information on the VS Fortran Version 2 language and library. Readers should have a basic understanding of Fortran. This document supersedes the IBM VS FORTRAN Version 2 Language and Library Reference, Release 4 (SC26-4221-4).

\section{IBM VS FORTRAN VERSION 2 PROGRAMMING GUIDE FOR CMS AND MVS, RELEASE 5 (SC26-4222-5)}

Contains information about how to code, compile and run VS Fortran programs on CMS and MVS. For application programming, you will necd to use both this book and the IBM VS FORTRAN Version 2 Language and Library Reference. Readers should have a basic understanding of Fortran. This document supersedes the IBM VS FORIRAN Version 2 Programming Guide for CMS and MVS, Release 4(SC26-4222-4).

\section{IBM 3084 FUNCTIONAL CHARACTERISTICS (GA22-70)88-4)}

Describes the components and functions of the IBM 3084 Processor Complex base model (Q) and improved model (QX), Readers should be familiar with System/370 architecture and have knowledge of virtual-storage and virtual-machine concepts.

\section{IBM 3380 DIRECT ACCESS STORAGE REFERENCE SUMMARY CARD (GX26-1678)}

Bases its capacity and speed data on information in the IBM 3380 Direct Access Storage Introduction (GC26-4491) and the IBM 3380 Direct Access Storage Direct Channel Attach Model C.J2 Introduction and Reference (GC26-4497).

\section{IBM 3800 PRINTING SUBSYSTEM CHARACTER SET CATALOG (GA26-1656)}

Provides a graphic representation of each character contained in every character set supplied with the IBM 3800 Printing Subsystem. This Catalog is for system programmers, application programmers, or other persons responsibie for designing or modifying characters to be used by the IBM 3800 printer. The Catalog assumes a basic understanding of how characters are generated for the IBM 3800 printer, as defined in the reference manual or programmer's guides.

\section{IBM 3800 PRINTING SUBSYSTEM OPERATOR'S GUIDE (GA26-1634)}

Provides opcrating instructions for the IBM 3800 Printing Subsystem. These instructions include descriptions of the controls, switches, and indicators used for initial 
sctup, running and error recovery. This publication is a reference guide for the operator who has recelved training in the IBM 380() printer operation, It assumes that the user of this document has a basic knowledge of printer $1 / 0$ operation. 'The publication is for the operators who will perform the basic sctup, operation, error recovery, and clcaning tasks associated with the IBM $38(x)$.

\section{IBM 380) PRINTINC SUBSYSTEM PROGRAMMLR'S GUIDE (GC26-3846)}

Provides planning and conversion information for the IBM 380() Printing Subsystem. The intended audience is dala-processing managers, system plannors, and systom programmess planning to incorporate an IBM 380() into computer installations, Information on how to use the IBM $38(x)$ is for application programmers and system programmers who will use the IBM 3800 Printing Subsystem. There is no prerequisite reading for this publication, although a basic knowledge of computers and system printers is assumed.

\section{IBM 3803 MODEL, 1, IBM 3420 MODELS 3, 5, AND 7 MACINETIC 'TAPE SUBSYSTEMS COMPONEN'T DESCRIPIION (GA32-0020)}

Describes the IBM 3803 Model 1 Tape Control and the IBM 3420) Models 3, 5, and 7 Magnetle Tape Units. The IBM $38(03$ Model 1 Tape Control provides communication between the tape units and the system $1 / O$ interface. The IBM 3420 Models 3, 5, and 7 tape units read and write one-half inch magnetic lape at 75, 125, and 200 inches per second, respectively. All three basic tape units read and write ninc-track, 1600 bytes per inch (bpi), phase-cncoded format. Nine-track, 800 bpi NRZI recording mode is available as a feature on all models. Seven-track, 556/8() bpl NRZI recording mode is an optional leature on all models. Readers should be familiar with the contents of IBM System/360 Principles of Operation (GA22-6821), especially the sections on $1 / 0$ opcrations, System/37() users should also refer 10 /BM System/370): Principles of Operation $(\mathrm{GA22}-7()()())$.

\section{IBM 3803 MODEL, 2, IBM 3420 MODELS 4, 6, AND 8 MACNETIC TAPL SUBSYSTHMS COMPONLNT DESCRIPTION (GA32-0021)}

Describes the IBM 3803 Model 2 Tape Control and the
IBM 3420 Models 4, 6, and 8 Magnotic Tape Units. The IBM 38()3 Model 2 Tape Control provides com. munication between the tape units and the system $1 / 0$ Interface. The IBM 3420 Models 4, 6, and 8 tape units read and writo one-half inch magnetic tape at 75, 125, and 200 inches per second, respectively. Readers should be familiar with IBM System/360 Principles of Operation (GA22-6821), especially the sections on I/O operations, and IBM System/370: Princlples of Opera. tion (GA22-7(000), espectally the section on $1 / O$ operations.

\section{IMSL LIBRARIES EDITION 10.0 UPDA'TE GUIDE (8709)}

Highlights information that will be useful during the transition to the new Edition 10.0 libraries. The new libraries are organized into three catogories: general applicd mathematics, statistics, and special functions. The first part of this report describes the general features of the new libraries. Next is a discussion of the specific additions and entancements to each chapter in the new Edition 10.0 libraries, with specific references to the carlier edlitions. The third part explains the llbrary interface utilities a set of programs that you may use while converting your programs to the now libraries). Tables at the back of the report list all of the old and new routine names with additional information.

\section{IMSL LIBRARY REFERENCE MANUAL}

Describes in detall (in seven volumes) the IMSL library, an extensive collection of mathematical and statistical subroutines written in Fortran. The discussion includes subroutine descriptions as well as examples of usage.

\section{INDICXER: A PROGRAM TO PRODUCE A QUALIITY INDEX (TM 299)}

Describes INDEXER, a computer program to assist in the production of a high-quality index for a document. Entries supplied by the user are assembled with the specifications provided by up to two dozen parameters. Included in this document are operating instructions, guidelines on the choice of parameters, and a sample index. 


\section{IN'TRODUCING THE IBM 3800 PRINTING SUBSYSTIEM AND I'IS PROCRAMMING} (GC26-3829)

Introduces bricfly the IBM $38(0)$ Printing Subsystem and its programming support. The IBM $38(0)$ Printing Subsystem is a non-1mpact, high-speed, genoralpurpose system printer that uses an olectrophotographic process to print on single-ply computer output paper. The Intended audionce is data processing installation managers and system analysts planning for tho IBM 3800 ) and others secking an introduction to it. Now features avallablo with the IBM $380($ ) are (1) printing with any tivo (or any four if optlonal charactor goneration storage is added) of 20 different character sots intermixed for the same datasct, (2) changing these character sets botween datasets without operator intervention (character sets are avallable in 10,12, and 15 pitch), (3) printing with a vertical line spacing of 6,8 , or 12 lines per inch or an intermlx of these on the same page, and (4) printing multiple coples on single ply under program control. Forms overlay permits printing of specializad forms as data is being printed. Copy modification pormits changing or suppressing printing of selected data from copy to copy when multiple copies are being printed. Graphle character modification allows the substitution or extension of graphic characters in an already-defined character set.

\section{INTRODUCING 'THE SAS SYS'TEM, VERSION 6, FIRST EDITION (1-55544-449-0)}

Describes the Sutistical Analysis System (SAS), programming with the SAS Display Manager System, accossing data, managing data, prosenting data in reports and with graphics, and bullding applications. This document supersedes the SAS Introductory Guide $(0-917382-73-(0)$.

\section{IN'T'RODUCTION TO IBM DIRECT'ACCESS ST'ORAGE DEVICLS AND ORGANIZATION METHODS ((GC20) 1649)}

Introduces the reader to the Direct-Acecss Storago Devices and their Control Units, and the data set organ" izations supported by OS/VS and DOS/VS. There is no discussion about the various macros used by the different access methods.

\section{INTRODUC'TION 'TO JVS3 (GC28.06,07)}

Contains Introdictory Information about JES3, one of the primary job ontry subsystoms of OS/VS2. One cill order JES3 as a soparate componont of OS/VS2, The reader should have a basic knowledge of programming systems, such as OS/MV'T or OS/VS2. Althought this publication is destgned to be read from cover to cover, the chapters are genorally independent and catin bo reforred to in any order. Tho "Introduction" presents highlights of JES3, whilo "JES3 Concopts" prosents JES3 as a subsystom and oxplains how a job flows through the JES3 complex. Subsecguent chapters dilscuss "JES3 Scrvices," "Installation Interfaces with JES3," "MVS Job Processing Sorvicos Used by JES3," "Recovery Procedures and Service Alds," "Planning Considerations," and "JES3 System Conflguration Options."

\section{IN'TRODUCTION TO MA'TERIALS AND SLRVICIES TRACKING A'T ANL (ANL/I'M 448)}

Offers a general overviow of Materials and Services Tracking (MAST) and explains how MAST comploments other financial systems at Argonne, It focuses on objectives, benefits, and functions of MAST, This Introduction does not teach you how to use MAST' (information on prompting scejuences, menus, ck, is avallable when you enroll as a MAST user or attend the CTD class for MAST usors) but does provide sufficient information for you to determine If and how the use of MAST can benefit your organization. This document is for the financial user community at $\mathrm{ANL}$. Administrative or executive assistants who are responsible for reviowing financtal reports, clorical or sccretarial staff who enter data or request reports, and managers or employecs performing planning and tracking activities are all part of this community.

\section{IN'TRODUCTION TO THE DESIGN, CREATION AND USE OF DATABASES USING PROLOG;}

Explains how to design and construct dalabases by using Prolog. This pamphlot covers the three distinct steps of this process by focusing on specific databases. This pamphlet assumes that readers have some knowledge of Prolog. 


\section{IN'T'RODUC'TION 'TO VIR'TUAL STTORAGE IN SYS'TIM/370 S'I'UDLEN'I 'I'LX'T' (GR20-4260)}

Explains the consepts of dystamic relocition, dynamic address translation, and virlual storage and defines all related terminology, 'The student text describes a theo. retical virtual storage system that is closely rolated to the implementation of OS/VS and DOS/VS, and it then describes how virtual storage is implemented in the OS/VS and DOS/VS systems on System/370.

\section{IN'TRODUC'IORY MACSYMA DOCUMENTATION: A COLLECTION OF PAPERS}

Describes how to access the MACSYMA system as it exists at the Laboratory for Computer Sclence at the Massachusetts insiltute of Technology and how to interatet with the environment in which it exists (t.e., operating system, monitor, editor, and other programs), The document is divided into three parts: (1) "An Introduction to I'T'S for the MACSYMA User," (2) "I'I'S Easy, Onico ITS Explained," and (3) "MACSYMA Primer."

\section{INVESTIIAATION OF GOSIP 'TECHNOLOGY A'T' ANL. (ANL/T'M 499)}

Describes testing of Open Systems Interconnection (OSI) products conducied at Argonne National Laboratory. Argonne used Sun, IBM, and Cisco hardware platlorms and evaluated various soltware packages that implement file transfer and gateway applications. This dexument briefly discusses the OSI model and Government Open Systems Interconnection Profile (GOSIP) compliantec and presents technical details on OSI addressing and routing. This document discusses the relationship of this testing to other OSI activities at Argenne and to activities of the national networking community. Also mentoned is the relationship of DEChet Phase V transition issues.

\section{ISSCO-(BKS COMMAND REFERENCE GUIDE}

Provides users with a quick reference tool that briefly describes the Graphics Kernel Standard (CKS) and the Integrated Software Systems Corporation (ISSCO)-GKS implementation and then lists the 191 user-callable ISSCO.GKS routines and functions. This document is for programmers who are well versed in computer graphics princlples; it is not a primer or text.

\section{ITE 4 TELEPHONE USER GUIDE (ANL/TM 471)}

Describes the Integrated Terminal Equipment (ITE) 4 single-line olectronic telephone and gives instructions for placing calls and using features. The ITE 4 can be used for simultaneous volce and data communications.

\section{ITE 12 TELEPHONE USER GUIDE (ANL/TM 472)}

Describes the ITE 12A, ITE 12P, and ITE 12S telephones and gives instructions for placing calls and using the ITE features. The ITE 12A, 12P, and $12 \mathrm{~S}$ telephones are electronic multiline sets that are compatible with data interface units for simultaneous volce and data communications.

\section{ITE 24 TELEPHONE USER (GUIDE (ANL/TM 473)}

Describes the ITE 24 telephone and gives instructions for placing calls and using the ITE features. The ITE 24 telephone is an electronte multiline set that is compatible with data interface units for sitmultancous voice and data communications.

\section{JES3 COMMANDS (SC23-0045)}

Provides some general information about the Job Entry Subsystem 3 (JES3) and documents the commands used to operate JES3. Any JES3 complex that runs MVS/370 will need this book. It is for use by JES3 operations staff and system programmers or by anyone else who is responsible for controlling JES3 and the input, job scheduling, networking, and output functions that JES3 provides for MVS. Note that this document explains the INQUIRE and MODIFY commands, many of which are usable from Wylbur, CMS, and RJP stations.

\section{JES3 COMMANDS: REFERENCE SUMMARY} (SX23-0007)

Summarizes Information from JES3 Commands (SC23-0045) and JES3 System Programming Library: Diagnosis (LC28-1369). 


\section{JES3 MESSAGES (GC23-0044)}

Lists the messages produced by the IBM-supplied JES3 component of the operating system, explains the causes of these messages, describes the accompanying actions by the operating system, and suggests appropriate responses.

\section{KERMIT USER GUIDE}

The Columbia University Center for Computing Activittes has designed the Kermit file transfer protocol for users of DEC and IBM interactive systems and PC users. This new edition covers new releases on many Kermil implementations and provides descriptions of now facillites (like login scripts). A CTD addendum covers Argonne changes to Kermit.

\section{KERMIT-MS FOR IBM PERSONAL COMPUTER, VERSION 3.11 (3 1/2-INCH DISKETTE)}

Is a single-sided double-density $31 / 2$-inch diskette for the IBM Personal Computer. This diskette contains the MS-DOS Kermit program and much-improved ANLwritten scripts for accessing the IBM and VAX clusters. This diskette supersedes the Kermit-MS for IBM Personal Computer, Version 3.01 diskette.

\section{KERMIT-MS FOR IBM PERSONAL COMPUTER, VERSION 3.11 (5 1/4-INCH DISKETTE)}

Is a single-sided double-density $51 / 4$-inch diskette for the IBM Personal Computer. This diskette contains the MS-DOS Kermit program and much-improved ANLwritten scripts for accessing the IBM and VAX clusters. This diskette supersedes the Kermit-MS for IBM Personal Computer, Version 3.01 diskettc.

\section{KWIC INDEX AND CROSS REFERENCE CHARTS (TM 261)}

Provides quick manual information retrieval. A KeyWord-in-Context lists the contents of the ANL Mathematical Subroutine Library (AMDLIB), A cross reference Chat shows the core storage requirement and the inter-rclationships among the routines in AMDLIB.

\section{LAPACK WORKING NOTE NO. 6: 'TOOLS TO AID IN THE ANALYSIS OF MEMORY ACCLSS PA'TTERNS FOR FOR'TRAN PROGRAMS (ANL/MCS.TM-120)}

Discusses MAP tools that provide an "animated" view of the momory activity during execution. Those tools allow rescarchers to play back a program's exccution, to study how an algorithm uses memory, and to experlmont with different memory hierarchy schemes and to observe their effects on the program's flow of data. This paper is avallable though the Mathemalics and Computer Science Dlvision (extension 2-7222).

\section{LATEX USER'S GUIDE AND REFERENCE MANUAL (0-201-15790-X)}

Describes the I. aTeX document preparation system, which is a special version of Donald Knuth's TeX program. TeX is a sophisticated program designed to produce high-quality typesetting, especially for mathematical text. LaTeX adds to TeX a collection of commands that simplify lypesetting by letting the user concentrate on the structure of the text rather than on formatting commands. This document explains how to prepare a LaTeX input file. Chapter 1 discusses the philosophy underlying LaTeX. Chapter 2 explains how to handle most simple documents. Chapter 3 describes logical structures for handling a varicty of formatting problems. Chapter 4 contains features cspecially useful for large documents. Chapter 5 describes the visual formatting of text. Chapter 6 explains how to deal with errors. The four appendixes cover topics including SLITCX (a version of LaTeX for making slides), a bibliographic database for use with BIBTeX, a compact description of all LaTeX's features, and TeX cominands that are not described in this book.

\section{LECTURES ON SPEAKEASY}

Provides information on Speakeasy. For individuals that have never used a computer, Speakeasy is an casy way to start. After the first one-hour lecture, the user will be able to do just about anything that can be done with a pocket calculator and will have results printed in neat tables. Compared to APL, Speakeasy is easicr to learn, but not as powerful or as compact. Compared to Fortran, PL/I, and Basic, Speakeasy works direclly with arrays, so many statements dealing with DIMENSIONS, loops, and output can be omitted. The first lecture is an introduction. Other lectures are selfcontained. After the first lecture, the others can be lak- 
en in any order. Some users may find the first lecture is sufficient for their needs. Others may pick and choose, depending on what they want the computer to do for them.

\section{LINPACK USF,R'S GUIDE (0-89871-172-X)}

Provides information on LINPACK, a collection of Fortran subroutines that analyze and solve various systems of simultaneous linear algebraic equations. The subroutines are designed to be completely machine independent, fully portable, and to run at near optimum efficiency in most operating environments.

\section{MACSYMA REFERENCE MANUAL VOLUME I}

Constitutes with Volume II a complete reference for the principal features of MACSYMA, a large computer programming sysiem written in LISP and used for performing symisolic as well as numerical mathematical manipulations. With MACSYMA, users can differentiate, integrate, take limits, solve systems of linear or polynomial equations, factor polynomials, expand functions in Laurent or Taylor series, solve differential equations (using direct or transform methods), compute Poisson series, and manipulate matrices and tensors. MACSYMA has a language similar to ALGOL-60 to permit users to write their own programs for transforming symbolic expressions. This document is not a tutorial; those unacquainted with certain concepts of computer programming will find this document difficult on first reading. DOE-MACSYMA is a variation of the original MACSYMA that is available on the central VAX 8700 computer.

\section{MACSYMA REFERENCE MANUAL VOLUME II}

Constitutes with Volume I a complete reference for the principal features of MACSYMA, a large computer programming system written in LISP and used for performing symbolic as well as numerical mathematical manipulations. With MACSYMA, users can differentiate, integrate, take limits, solve systems of linear or polynomial cquations, factor polynomials, expand functions in Laurent or Taylor series, solve differential equations (using direct or transform methods), compute Poisson series, and manipulate matrices and tensors. MACSYMA has a language similar to ALGOL- 60 to

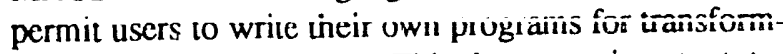
ing symbolic expressions. This document is not a tutorial; those unacquainted with certain concepts of computer programming will find this document difficult on first reading. DOE-MACSYMA is a variation of the original MACSYMA that is available on the central VAX 8700 computer.

\section{MANAGER'S GUIDE TO THE ARGONNE NJE EMULATOR (ANL/TM 479)}

Describes the Argonne NJE Emulator, which allows VAX/VMS systems to communicate with IBM computers with the VM/RSCS, MVS/JES2, MVS/JES3, or VSE/POWER operating systems or either CDC computers having the NJEF product.

\section{MANUAL PURCHASE ORDER CLOSING (ANL/TM 480, REVISION 1)}

Describes how to close a purchase order manually. Readers should have a working knowledge of the Automated Materials Payables System (AMPS). This document supersedes Manual Purchase Order Closing (ANL/TM 480).

\section{MASS-11 WORD PROCESSING}

Is a boxed set containing three separate documents: Mass-11 Word Processing: Getting Started, Mass-11 Word Processing: Pocket Reference Guide, and Mass-11 Word Processing: VAX/VMS Reference Manual. These documents describe Version 8.0 of Mass-11. This document supersedes the Mass-11 Word Processing VAX/VMS Reference Manual (Version $7-A$ ).

\section{MATHEMATICA: A SYSTEM FOR DOING MATHEMATICS BY COMPUTER (0-201-51507-5)}

Describes all the capabilities of the Mathematica system and assumes no prior knowledge of the system.

\section{MATRIX EIGENSYSTEM ROUTINES--EISPACK GUIDE RXTENSION--51 (3-540-08254-9)}

Supplements the earlier Volume 6 in this series; together they provide guidance for the complete second release of the EISPACK Figensystem Package. The stress in this book is on four additional problem classes: the symmetric band eigenproblem, the generalized 
symmetric band eigenproblem, the generalized real eigenproblem, and the singular value decomposition of a rectangular matrix and solution of an associated linear least squares problem.

\section{MATRIX EIGENSYSTEM ROUTINES--EISPACK GUIDE--6 (3-540-06710-8)}

Is a user guide to a portion of EISPACK, a systematized collection of subroutines that compute the eigenvalues and/or eigenvectors of matrices. This volume covers six classes of matrices: complex general, complex Hermitian, real general, real symmetric, real symmetric tridiagonal, and special real tridiagonal. The National Activity to Test Software (NATS) Project supports and has certified these subroutines.

\section{MICROSOFT LEARNING DOS (089-015V200-3.5)}

Is an online course in a $31 / 2$-inch floppy disk format that describes how to use Microsoft DOS for IBM Personal Computers and PC compatibles. Microsoft Learning DOS replaces the Learning DOS $31 / 2$-inch diskette.

\section{MICROSOFT LEARNING DCS (089-015V200-5.25)}

Is an online course in a 5 1/4-inch floppy disk format that describes how to use Microsoft DOS for IBM Personal Computers and PC compatibles. Microsoft Learning DOS replaces the Learning DOS 5 1/4-inch diskette.

\section{MULTINET VERSION 3.0 MANUALS}

Consists of two volumes. The first volume includes the MultiNet NFS Client System Administrators' Guide, which describes the administration of the MultiNet Network File System (NFS) Client, and the MultiNet NFS Server System Administrators' Guide, which describes the administration of the MultiNet NFS Server. The second volume includes the MultiNet Users' Guide, which documents the user utilities and functionality of the MultiNet software, and the MultiNet Programmers' Reference Manual, which documents the programmers' interface to the MultiNet software.

\section{MVS BATCH COMPUTING AND DATA MANAGEMENT AT ANL (ANL/TM 400, REVISION 1)}

Describes the batch computing system running under MVS (Multiple Virtual Storage) on the IBM 3084 computer at Argonne National Laboratory. It serves as a guide to the Argonne MVS batch computing environment and recommends practices and modes of operation. It explains the terminology and conventions used at Argonne, describes some job control language (JCL) you will need to run your MVS batch jobs, and shows you how to manage your data files on disk and tape devices. This document supersedes MVS Batch Computing and Data Management at ANL (ANL/TM 400).

\section{MVS BATCH UTILITIES FOR FILE MANAGEMENT AT ANL (ANL/TM 412)}

Describes the operation and use of the MVS batch utilities and cataloged procedures for filc management available at Argonne's central IBM computing system. This document supersedes OS/MVT Batch Utilities at ANL (Technical Memorandum 374). Many of the utilities and procedures documented herein are alternatives to the standard IBM utilities documented in MVS/370 Data Administration Utilities, Release 1.2 (GC26-4065-3), while others provide functions not found in the standard IBM utilities. This document uses these approaches to present tools for file management: Wylbur commands, execs (Wylbur and CMS), batch utilities and cataloged procedures, and IBM utilities. In preparing this document, we have assumed that you have a working knowledge of cataloged procedure and IBM JCL usage. If you do not have such knowlcdge, read MVS Batch Computing and Data Management at ANL (ANL/TM 400, REVISION 1) for information on using cataloged procedures and IBM JCL.

\section{MVS/EXTENDED ARCHITECTURE JCL REFERENCE (GC28-1352-4)}

Lists the job control tasks needed to enter jobs into the operating system, to control the system's processing of jobs, and to request the resources needed to run jobs. To perform the tasks, programmers code job control statements. This document describes how to code these statements. Full explanations of the job control tasks are in the MVS/Extended Architecture JCL User's Guide (GC28-1351-4). This document is for system and application programmers who enter programs iniw the operating system. Users of this document should 
understand the concepts of job management and data management.

\section{MVS/EXTENDED ARCHITECTURE JCL USER'S GUIDE (GC28-1351-4)}

Describes the job control tasks needed to enter jobs into the operating system, to control the system's processing of jobs, and to request the resources needed to run jobs. To perform the tasks, programmers code job control statements. This document describes how to use these statements. This document is a user's guide, to be used when deciding how to perform job control tasks. It does not describe how to code the statements. For an introduction to the statements and for coding information, see the MVS/Extended Architecture JCL Reference (GC28-1352-4). This document is for system and application programmers who enter programs into the operating system. Users of this document should understand the concepts of job management and data management.

\section{MVS/EXTENDED ARCHITECTURE MESSAGE LIBRARY：JES3 MESSAGES (GC23-0062-4)}

Is for any JES3 complex that runs Multiple Virtual Storage/Extended Architecture (MVS/XA) or for any JES3 complex that runs both MVS/XA and MVS $/ 370$. This document is for JES3 operations staff, applications or system programmers, or anyone who is responsible for controlling JES3 or diagnosing problems in JES3.

\section{MVS/EXTENDED ARCHITECTURE MESSAGE LIBRARY: SYSTEM CODES (GC28-1157-6)}

Describes completion codes and wait state codes and the causes of uncoded wait states and loops. The description of most codes ends with a problem determination paragraph, which lists suggested actions as items in tables. This document is for operators, system programmers, and applications programmers.

\section{MVS/EXTENDED ARCHITECTURE MESSAGE LIRRARY: SYSTEM MESSAGES, VOLUME 1 ADY-IEB (GC28-1376-7)}

Contains descriptions of messages with prefixes ADY through IEB that many MVS/Extended Architecture operating system components issue.

\section{MVS/EXTENDED ARCHITECTURE MESSAGE LIBRARY: SYSTEM MESSAGES, VOLUME 2 IEC-ITV (GC28-1377-7)}

Contains descriptions of messages with prefixes IEC through ITV that many MVS/Extended Architecture operating system components issue.

\section{MVS/EXTENDED ARCHITECTURE OPERATIONS: JES3 COMMANDS (SC23-0063-3)}

Is for any JES3 complex that runs Multiple Virtual Storage/Extended Architecture (MVS/XA). This document is for JES3 operations staff and system programmers or for anyone who is responsible for controlling JES3 and the input, job scheduling, networking, and output functions that JES3 provides for MVS. This document provides some general information about JES3 and documents the commands used to operate JES3.

\section{MVS/SYSTEM PRODUCT VERSION 2 RELEASE 2 GENERAL INFORMATION (GC28-1500-3)}

Contains gencral overview and planning information for Version 2 Release 2.0 (and all subsequent releases). This document is for installation managers and system programmers who are considering installing either of these products. Readers of this document should have a background in MVS.

\section{MVS/370 DATA ADMINISTRATION MACRO INSTRUCTION REFERENCE (GC26-4057)}

Contains information needed by system and application programmers to use the data management access method functions of MVS/370 Data Facility Product 5665-295. This document describes and defines the data management macro instructions--except for the Virtual Storage Access Method (VSAM)--available in the assembler language.

\section{MVS/370 DATA ADMINISTRATION UTILITIES, RELEASE 1.2 (GC26-4065-3)}

Describes how to use the MVS/370 Data Facility Product utility programs to manipulate system and user data and datasets. Readers should be familiar with JCL, 
data management, and virtual storage management. This document supersedes MVS/370 Data Administration Utilities (GC26-4065-2).

\section{MVS/370 INTEGRATED CATALOG ADMINISTRATION ACCESS METHOD SERVICES REFERENCE, RELEASE 1.2 (GC26-4051-2)}

Is for catalog administrators and VSAM system programmers. This document contains reference information about the access method services commands used to manipulate integrated catalog facility catalogs and VSAM datasets. It gives the syntax, a brief description, and examples of each access method services command used with integrated catalog facility catalogs and the objects cataloged in them. For information on the use of commands related to integrated catalog facility catalog format and structure, see the Catalog Administration Guide. For information on the use of commands related to VSAM dataset format and structure, see the VSAM Administration Guide.

\section{MVS/370 JCL REFERENCE (GC28-1350-3)}

Lists the job control tasks needed to enter jobs into the operating system, control the system's processing of jobs, and request the resources needed to run jobs. To perform the tasks, programmers code job control statements. This publication describes how to code these statements that consist of job control language (JCL) statements, Job Entry Subsystem 2 (JES2) control statements, and Job Entry Subsystem 3 (JES3) control statements. This document is a reference book for use while coding statements. This document supersedes the MVS/370 JCL Reference (GC28-1350-2).

\section{MVS/370 JCL USER'S GUIDE (GC28-1349-3)}

Describes the job control tasks needed to enter jobs into the operating system, control the system's processing of jobs, and request the resources needed to run jobs. To perform the tasks, programmers code job control statements. This document describes how to use these statements, which consist of job control language (JCL) statements, Job Entry Subsystem 2 (JES2) control statements, and Job Entry Subsystem 3 (JES3) con. trol statements. This revision contains maintenance updates for MVS/System Product Version 1, Release 3.6. This document is a user's guide, for use when users must dacide how best to perform job control tasks; it does not describe how to code the statements. For an introduction to the statements and for coding information, see the companion book, MVS/370 JCL Reference (GC28-1350-3).

\section{MVS/370 LINKAGE EDITOR AND LOADER USER'S GUIDE (GC26-4061)}

Provides application programmers with the information necessary to use the MVS/370 Data Facility Product linkage editor and loader to prepare the output of a language translator for execution. This document also provides information on the operation and use of the linkage editor and loader for the system programmer responsible for installing and maintaining the operating system.

\section{MVS/370 MESSAGE LIBRARY: SYSTEM CODES (GC38-1008)}

Lists, explains, and suggests appropriate responses for the system codes and state codes produced by IBMsupplied components of MVS. The codes are presented in alphanumeric order. This document supersedes OS/VS Message Library: VS System Codes (GC38-1008).

\section{MVS/370 MESSAGE LIBRARY: SYSTEM MESSAGES VOLUME 1 AHL-IEB (GC28-1374-4)}

Contains descriptions of messages with prefixes AHL through IEB. The messages are grouped together in sections by their three-letter prefixes, which identify the components producing the message. Each section begins with the component name, the audience for the message, the message format, publications related to or referenced by the message, and other useful information. These descriptions explain why the component issued the message, give the action of the operating system, and suggest responses for the applications programmer, system programmer, or operator to make.

\section{MVS/370 MESSAGE LIBRARY: SYSTEM MESSAGES VOLUME 2 IEC-ISG (GC28-1375)}

Contains descriptions of messages with prefixes IEC through ISG. The messages are grouped together in sections by their threc-letter prefixes, which identify the components producing the message. Each section hegins with the component name, the audience for the 
message, the message format, publications related to or referenced by the message, and other useful information. These descriptions explain why the component issued the message, give the action of the operating system, and suggest responses for the applications programmer, system programmer, or operator to make. This volume also includes problem determination tables. These tables list the suggested actions. This volume also includes a message-to-module table. This table lists the operating system modules that detect the need for the message, that issue the message, and that contain the message text.

\section{MVS/370 VSAM ADMINISTRATION GUIDE (GC26-4066)}

Describes the use of the Virtual Storage Access Method (VSAM). This document is for programmers who use access method services commands, VSAM macro instructions, and JCL to process VSAM datasets. Readers should have a programming background that includes catalog administration, job control language, and principles of operation.

\section{NAG FORTRAN LIBRARY INTRODUC'TORY GUIDE MARK 13 (1-85206-046-8)}

Serves as an extensive pointer to the main Numerical Algorithms Group (NAG) Fortran Library, Mark 13. For each chapter in the NAG library, this document provides background advice on the subject area covered, recommendations on the choice and use of routines, and a summary of the purpose of each routine.

\section{NAG FORTRAN LIBRARY IN'TRODUCTORY GUIDE MARK 14 (1-85206-054-9)}

Serves as an extensive pointer to the main Numerical Algorithms Group (NAG) Fortran Library, Mark 14. For each chapter in the NAG library, this document provides background advice on the subject area covered, recommendations on the choice and use of routines, and a summary of the purpose of each routine.

\section{NAG FOR'TRAN MINI MANUAL MARK 12 (1-85206-033-6)}

Serves as an extensive pointer to the main Numerical Algorithms Group (NAG) Fortran Library, Mark 12. For cach chapter in the NAG library, this document provides background advice on the subject area cov. ered, recommendations on the choice and use of routines, and a summary of the purpose of each routine.

\section{NCSA HDF CALLING INTERFACES AND UTILITIES, VERSION 3.1}

Is for users who are working on an application that involves the use of the National Center for Supercomputing Applications (NCSA) Hierarchical Data Format (HDF) files. To use HDF software, you need access to one of the following computer systems: a Cray with UNICOS, a Silicon Graphics system with Unix, an Alliant with CONCENTRIX, a Sun System 3 with Unix, a VAX terminal with Unix, a VAX with VMS, a Macintosh with MacOS, or an IBM PC with MS-DOS or compatible model.

\section{NCSA IMAGE FOR THE COLOR MACINTOSH, VERSION 3.0}

Describes how to use the the National Center for Supercomputing Applications (NCSA) Image color imaging program on an Apple Macintosh with $256 \mathrm{col}$ or capabilities. This document is divided into five chapters and one appendix. Chapter 1 provides an introduction to NCSA Image and a bricf tutorial to start the user on the program. Chapter 2 explains how NCSA Image reads and displays data files. Chapter 3 explains how windows function in the program, how to incorporate palettes other than the default palette, and how to save changes to the color palette. Chapter 4 describes the procedures to perform imaging manipulations and data analyses in NCSA Image. Chapter 5 provides brief descriptions and references for each of the tools and commands used in NCSA Image. Appendix A presents the various error messages you may encounter in using NCSA Image.

\section{NCSA PC SHOW FOR THE IBM PC, VERSION 1.0}

Is a 5 1/4-inch diskette containing PC Show for the IBM Personal Computer and compatibles.

\section{NCSA PC SHOW FOR THE IBM PC, XT, AT, AND PS/2 USER'S GUIDE}

Describes how to use the National Center for Supercomputing Applications (NCSA) PC Show on the IBM PC, XT, AT, and PS/2 computers. Chapter 1 provides 
a brief discussion of the commands used to begin PC Show, command line parameters, and methods of exiting the program. Chapter 2 delineates the various com. mands that concern the display of animations or multiple images. Chapter 3 describes both the characteristics of various palettes and the commands that permit the manipulation of palettes.

\section{NCSA TELNET FOR PC VERSION 2.2TN AND VERSION 2.2D (UD-99 US-14)}

Includes the enhancements added by Clarkson University to Version 2.2 of the National Center for Supercomputing Applications (NCSA) Telnet, which provides interactive access from an IBM PC or compatible to Telnet hosts on Transmission Control Protocol/ Internet Protocol (TCP/IP) networks. This document is organized into six chapters and three appendixes. Chapter 1, "Starting and Quitting NCSA Telnet,"" describes how to start NCSA Telnet and how to open and close a connection between your PC and one remote host. Chapter 2, "Introduction to Managing Systems," introduces NCSA Telnet's capability for multiple connections. Chapter 3, "More About Managing Sessions," discusses multiple sessions in detail. Chapter 4, "File Transfer," outlines procedures for transferring files between a PC and a Telnet host. Chapter 5, "Tektronix 4014 Emulation," discusses NCSA Tclnet's ability to emulate a Tektronix 4014 graphics terminal. Chapter 6, "Installation and Configuration," contains information for system administrators (and other experienced users) to use when installing and customizing a system. The three appendixes describe NCSA Telnct's error messages, commands available from the PC keyboard, and keywords for configuring file parameters. A CTD addendum describes local variances.

\section{NCSA TELNET FOR THE IBM PC VERSION 2.2TN AND 2.2D (3 1/2-INCH DISKETTE)}

Is a $31 / 2$-inch diskette containing version 2.2TN and 2.2D of Telnet for IBM Personal Computers and compatibles.

\section{NCSA TELNET FOR THE IBM PC VERSION 2.2TN AND 2.2D (5 1/4-INCH DISKETTE)}

Is a $51 / 4$-inch diskette containing version 2.2TN and 2.2D of Telnet for IBM Personal Computers and compatibles.

\section{NCSA TELNET FOR THE MACINTOSH, VERSION 2.4}

Describes how to use the National Center for Supercomputing Applications (NCSA) Telnet for Macintosh Version 2.4 on an Apple Macintosh to access Telnet hosts on Transmission Control Protocol/Internct Protocol (TCP/IP) networks. This document has cight chapters: "Getting Started," "Using the Keyboard," "Customizing the Environment," "Advanced Fcatures," "File Transfer," "Tcktronix 4014 Emulation," "Interactive Color Raster Graphics," and "System Administrator Information." The three appendixes describe NCSA Telnet's error messages, code to convert Unix /ete/hosts files, and procedures for obtaining NCSA software. A CTD addendum describes site-dependent features.

\section{NCSA X DATASLICE FOR THE X WINDOW SYSTEM, VERSION 1.0}

Explains how you should install and invoke the National Center for Supercomputing Applications (NCSA) X Dataslice, describes the basic file formats used in NCSA X DataSlice, describes options available in the Planes Along Axes window, outlines the options available in the Arbitrary Planes window, outlines the options available in the Cartesian Dicer window, and explains the procedures for loading and animating a saved 8-bit Raster image and for processing a scientific dataset.

\section{NCSA X IMAGE FOR THE X WINDOW SYSTEM, VERSION 1.0}

Describes the basic file formats used in the National Center for Supercomputing Applications (NCSA) X Image, explains the various data display options provided by NCSA X Image, describes palettes as they are used in NCSA X Image, and discusses the Animate feature of NCSA X Image.

\section{NETWORK ACCESS TO CICS: APPLE MACINTOSH}

Describes how you can access the Customer Information Control System (CICS) with your Apple Macintosh computer. 


\section{NETWORK ACCESS TO CICS: FULL SCREEN TERMINAL OR ASCII TERMINAL}

Describes how you can access the Customer Information Control System (CICS) with your full-screen or ASCII terminal. This brochure supersedes the December 1988 brochure.

\section{NETWORK ACCESS TO CICS: IBM PERSONAL COMPUTER}

Describes how you can access the Customer Information Control System (CICS) with your IBM Personal Computer.

\section{NONLINEAR OPTIMIZATION ROUTINES IN AMDLIB ('TM 297)}

Describes a set of six computer algorithms addressed to the unconstrained nonlinear optimization problem in AMDLIB, the subroutine library of ANL CTD. It discusses the strengths and weaknesses of this set of computer algorithms and summarizes in a decision chart. There is a description of the modular organization of the routines and the efforts made to systematize the software. The appendix gives the theoretical background for the set of algorithms.

\section{NSPCG (NON-SYMMETRIC PRECONDITIONED CONJUGATE GRADIENT) USER'S GUIDE (CNA-216)}

Describes the Non-Symmetric Preconditioned Conjugate Gradient (NSPCG) solver. NSPCG is a computer package currently installed on the Cray X-MP/18 that solves large sparse systems of lincar equations by various iterative methods.

\section{OBS WYLBUR REFERENCE MANUAL}

Is the basic reference document for Wylbur language users. It contains an extensive addendum summarizing ANL differences. This document consists of six sections. The "Introduction" provides a brief description of On-Line Business Systems (OBS) Wylbur. "The Wylbur Environment" describes and highlights the nature and behavior of Wylbur as seen by the user. This section introduces major concepts and considerations for use. It identifies features unique to various types of compatible terminal devices. The "Command Summary" provides a functional overview of the various types of Wylbur commands and a summary of the purpose of each command. "Detailed Command Description" describes the precise syntax and use of cach Wylbur command. "Wylbur Exccute (EXEC) Facilitics" presents an overview of the EXEC facilitics and a detailed explanation of the Preprocessor capability. This section defines available built-in functions and expression evaluation. "Wylbur Utilities" presents an overview (with details on use) of the utilities WLIBLIST, WUNPRESS, and WPRESS.

\section{OPERATING SYSTEM PL/I OPTIMIZING COMPILER: MESSAGES (SC33-0027)}

Lists all the messages that may be issued by the OS PL/I Optimizing Compiler and the associated transient library during processing of a PL/I program. Both the long and short forms of each message are listed where applicable. The messages are in three groups: compile-time messages (generated by the compiler during compilation of a PL/I source program), exccutiontime messages (generated by the transient library during execution of the compiled program), and prompter messages (generated by the compiler when it is used in a time-sharing environment). Where appropriate, explanations of the messages and suggested programmer responses are included.

\section{OS AND DOS PL/I LANGUAGE REFERENCE MANUAL (GC26-3977)}

Is a reference for the PL/I programmer. It is not a tutorial, but is designed for the reader who already has a knowledge of the language and who requires reference information to write a program that will be processed by the OS PL/I Optimizing Compiler.

\section{OS PL/I CHECKOUT AND OP'TIMIZING COMPILERS: KEYWORDS REFERENCE SUMMARY (SX33-6002)}

Summarizes the keywords and abbreviation of the OS PL/I Checkout and Optimizing Compilers kcywords. It also contains use of the keywords and examples. For further explanation, refer to the OS PL/I Checkout and Optimizing Compilers: Language Reference Manual. 


\section{OS PL/I CHECKOUT AND OPTIMIZING COMPILERS: TERMINAL COMMANDS AND COMPILER OPTIONS SUMMARY (SX33-6005)}

Contains a list of those commands most likely needed by the PL/I programmer working under CMS of VM/370 and TSO, It also contains the subcommands of the PLIC and PLICR commands and the subcommands of the CMS EDIT command.

\section{OS PL/I OPTIMIZING COMPILER: CMS USER'S GUIDE (SC33-0037)}

Provides the information required to use the OS PL/I Optimizing Compiler under CMS. Using the OS PL/I Optimizing Compiler under CMS allows PL/I source programs to be compiled, debugged, and executed from a terminal with the maximum efficiency. This document is for all programmers who use the OS PL/I Optimizing Compiler under CMS. It contains a brief tutorial introduction followed by reference data covering (1) the syntax and options of PLIOPT, the command that invokes the OS PL/I Optimizing Compiler; (2) the compile-time and execution-time options that can be used with the OS PL/I Optimizing Compiler; and (3) the restrictions that apply to PL/I when it is used under CMS.

\section{OS PL/I OP'TIMIZING COMPILER: EXECUTION LOGIC (SC33-0025)}

Describes the object module produced by the OS PL/I Optimizing Compiler and explains how the compiled code uses subroutines from the resident and transient libraries of the optimizing compiler. The topics covered include program initialization, storage management, input/output, error handling, and interlanguage communication. This document explains the use of storage dumps for debugging. This document is primarily for applications programmers who need an understanding of the processes of execution (e.g., to link $\mathrm{PL} / \mathrm{I}$ and Assembler language routines).

\section{OS PL/I OPTIMIZING COMPILER: GENERAL INFORMATION MANUAL (GC33-0001)}

Introduces and describes the compiler and the associated resident and transient libraries. The information is provided to use as a planning aid and to assist both existing and prospective users of PL/I. Topics covered in this document include the design philosophy and performance of the compiler, the optimization facilities provided, comparison with the PL/I (F) compiler, machine and system requirements of the compiler, summary of the $\mathrm{PL} / \mathrm{I}$ language implemented, and a bibliography of related publications. The reader should be familiar with PL/I and the operating system.

\section{OS PL/I OPTIMIZING COMPILER: PROGRAMMER'S GUIDE (SC33-0006)}

Is a companion volume to $O S P L / L$ Optimizing and Checkout Compilers: Language Reference Manual (SC33-0009) and OS/360 Time Sharing Option: PL/I Optimizing Compiler (SC33-0(29). The three documents form a guide to the writing and exccution of $\mathrm{PL} / \mathrm{I}$ programs using the optimizing compiler and the associated resident and transient libraries in the batch and timesharing environments of the IBM operating system. This document is concerned with the relationship between a PL/1 program, the optimizing compiler, and the opcrating system. It explains how to compile, linkedit, and executc a program in a batch environment and introduces job control language, the linkage editor, and the loader, data management, and other operating system features that a PL/I programmer may requirc.

\section{OS/VS DOS/VS AND VM/370 ASSEMBLER LANVIJAGE (GC33-4010)}

Provides the information necessary for programmers responsible for designing and writing programs in either the OS/VS or DOS/VS assembler language and for programmers running their assembler program under the $\mathrm{VM} / \mathrm{System}$. The assembler language is a symbolic programming language that allows the use of alphabetic and numeric characters to represent machine instructions and related data. Using symbolic language instead of the binary $1 \mathrm{~s}$ and 0 s required when coding in machine language makes coding easier to write, understand, and change. The assembler can process a program written in assembler language to produce output in machine language suitable for linkediting and subsequent exccution. The book is divided into four parts: Part I ("Coding and Structure"), Part II ("Function and Coding of Machine Instructions"), Part III ("Functions of Assembler Instructions"), and Part IV ("The Macro Facility"). The first part describes the statements, character set, terms, literals, and expressions that make up the assembler language, and gives the rules and conventions for writing code. The second part describes the alignment, statement formats, mnemonic operation codes, and operand entries of machine instructions. The third part describes the assembler instructions used for program definition and controlling the assembler. 
The fourth part introduces users to the macro facility and then describes the macro definition, the macro instruction, and the conditional Assembly language (a language that can alter the contents and sequence of the oulput produced by the assembler).

\section{OS/VS TAPE LABELS (GC26-3795-3)}

Explains, for programmers and operations analysts, how OS/VS processes magnetic tape labels. The four main sections of this document describe the specifications and processing of tapes with IBM standard labels, tapes with American National Standard labels, tapes with nonstandard labels, and unlabeled tapes.

\section{OS/VS VM/370 ASSEMBLER PROGRAMMER'S GUIDE (GC33-4()21)}

Complements OS/VS DOS/VS VM/370 Assembler Language (GC33-4010) This document is for the programmer writing assembler language programs to be run in OS/VS and/or VM/370). This document contains the following major sections: "Job Control Statements To Run the Assembler," "Including Macro Definitions in a Library," "Interpreting the Assembler Listing," "Programming Considerations," and "Assembler Language Programming under CMS." Other topics, covered in appendixes, are "Assembler Data Sct Requirements," "The Object Module Produced by the Assembler," and "Invoking the Assembler Error Messages." Prerequisitc reading is Introduction to $O S$ (GC28-6534), VM/370): Introduction (GC20-1800), and the OS/VS DOS/VS and VM/370 Assembler Language (GC33-4010).

\section{OS/VS2 MVS OVERVIEW (GC28-0984)}

Describes the main features of MVS. It explains cach of these features and described the flow of work through the major parts of the system. This document is for a general audience, but some knowledge of operating systems is necessary. There are no prerequisites.

\section{OS/VS2 MVS RACF MESSAGES AND CODES (SC38-1014-10)}

Lists the messages, abend codes, and Remote Access Control Facility (RACF) manager return codes produced by Version 1, Release 8.1 or 8.2 of the IBM program product RACF, program number 5740-XXH. This document supersedics the Resource Access Con- trol Facility (RACF) Messages and Codes

(SC38-1014).

\section{OS/VS2 MVS SYSTEM PROGRAMMING LIBRARY: SERVICE AIDS (GC28-0674)}

Explains how, why, and when to use IBM service aids programs for MVS for diagnosing and fixing failures in system or application programs. Each service aid is explained in a separate chapter, and the chapters are arranged in alphabetical order. This document is for system programmers, system operators, and IBM program support representatives.

\section{OS/VS2 SUPERVISOR SERVICES AND MACRO INSTRUCTIONS (GC28-1114)}

Is mainly for the programmer coding in assmbler language. This document describes how to use the services of the supervisor, the macro instructions used to request these services, and the linkage conventions used by the control program to provide these services. Part I of this document ("Supervisor Services") provides explanations and aids for using the facilities available through the supervisor. Part II ("Macro Instructions") provides coding information.

\section{OS/VS2 SYSTEM PROGRAMMING LIBRARY: DATA MANAGEMENT (GC26-3830-4)}

Provides information on how to modify and extend the data management capabilities of the MVS system control program. This document is for system programmers.

\section{OVERVIEW OF THE NON-PROGRAMMATIC COST PROJECTION PROTOTYPE} (ANL/TM 440)

Is for those people who plan and track human and matcrial costs for the organizations of the Laboratory. This document does not teach the reader how to budget at ANL; it does describe the Non-Programmatic Cost Projection prototype (CostPro) and the role it can play in the budgeting process of the non-programmatic organizations. CostPro is a locally developed computerized system that provides uniform planning and tracking tools for the Laboratory; it also provides the flexibility to satisfy the variety of needs of existing non-programmatic organizations. 


\section{PARALLELIZING THE SDI ACCESS ALGORITHM FOR THE CONNECTION MACHINE-2 (ANL-89/41)}

Describes taking a prototype weapon-target accessibility algorithm (ACCESS) previously studied on a variety of shared-memory parallel computers and analyzing the implementation of that algorithm on a Connection Machine-2. This report provides benchmarks for the algorithm on CM-2 configurations with $16 \mathrm{~K}$ to $64 \mathrm{~K}$ physical processors, along with comparative timings from other parallel and sequential implementations.

\section{PATHWORKS FOR DOS USER'S HANDBOOK, VERSION 4.0 (AA.PAF7B-TK)}

Shows personal computer users how to use PATHWORKS with the disk operating system (DOS), This document describes what the PATHWORKS systcm is and how you can use PATHWORKS for DOS network services and applications. This document assumes that the user is familiar with personal computers and has some knowledge of DOS. This document supersedes the Guide for New Users (AA-PAF7A-TK).

\section{A PLAN FOR ADMINISTRATIVE COMPUTING AT ANL FY1989 THROUGH FY1991}

(ANL/TM 464)

Identifies the components of administrative computing at ANL, provides guidelines for development of new or enhanced systems, and outlines the procedures by which management determines the structure and organization of administrative computing systems. This document proceeds from the premise that administrative information is a Laboratory asset; therefore, the Laboratory must know the quality, characteristics, availability, costs, and location of information as it would any other asset. This document incorporates detailed summaries of specific projects proposed for the three-year span and broader recommendations for the course of administrative computing development over a ten-year span. This document includes a listing of all known administrative computing systems in use onsite. Charts of expenditures for both user-funded and Laboratoryfunded systems provide a complete statement of the Laboratory's financial commitment to administrative computing.

\section{A PLAN FOR ADMINISTRATIVE COMPUTING AT ANL FY1990 'THROUGH FY1992 \\ (ANL/TM 474)}

Identifies the components of administrative computing at ANL, provides guidelines for development of new or enhanced systems, and outlines the procedures by which management determines the structure and organization of administrative computing systems. This document proceeds from the premise that administrativo information is a Laboratory asset; therefore, the Laboratory must know the quality, characteristics, avallability, costs, and location of information as it would any other asset. This document incorporates detailed summaries of specific projects proposed for the threc-year span and broader recommendations for the course of administrative computing development over a ten-year span. This document includes a listing of all known administrative computing systems in usc onsitc. Charts of expenditures for both user-funded and Laboratoryfunded systems provide a complete statement of the Laboratory's financial commitment to administrative computing.

\section{A PLAN FOR ADMINISTRATIVE COMPUTING AT ANL FY1991 THROUGH FY 1993}

(ANL/TM 483)

Identifies the components of administrative computing at ANL, provides guidelines for development of new or enhanced systems, and outlines the procedures by which management determines the structure and organization of administrative computing systems. This document proceeds from the premise that administrative information is a Laboratory asset; therefore, the Laboratory must know the quality, characteristics, availability, costs, and location of information as it would any other asset. This document incorporates detailed summaries of specific projects proposed for the three-year span and broader recommendations for the course of administrative computing development over a ten-year span. This document includes a listing of all known administrative computing systems in use onsite. Charts of expenditures for both user-funded and Laboratory. funded systems provide a complete statement of the Laboratory's financial commitment to administrative computing. 


\section{A PLAN FOR ADMINIS'I'RA'TIVE COMPU'TING A'T ANL FY 1992 THIROU(iH FY1994 \\ (ANL.T'M 489)}

Idontifies the components of administrative computing at ANL, provides guidelines for developmont of new or enhanced systems, and outlines the procedures by which managemont detorminos the structure and organzation of administrative computing systems. This document proceeds from the premise that administrative Information is a Laboratory assed; therefore, the Laboratory must know the quality, characteristics, availability, costs, and location of information as it woukd any other asset. This document incorporates dotalled summaries of specifte projects proposed for the three-year span and broader recommendations for the course of administrative computing development over a ten-year span. 'This document includes a listing of all known adminkstrative computing systems in use onsite. Charts of expenditures for both user-funded and Laboratoryfunded systems provide a complete statement of the Laboratory's finuncial commitment to administrative computing,

\section{A PLAAN WOR SCIENTIIIC VISUALIZATION AT ANL (ANL/TM 476)}

Describes the topic of scientific visualization and a proposed scientific visuallization plan for Argonne. Chapter 1, "An Overview of Scicntific Visualization," describes the basic concepts of visualization and the benefits of scientific visualization to ANL scientists. Chapter 2, "Current Scientific Visualization at ANL," describes the current software and hardware configuration at ANL, and provides descriptions and photographs of different visualization projects. Chapter 3, "Projected Plan for Scientific Visualization at ANL," describes the planned scientific visualization facility at ANL.

\section{PL/I PRIMLR: S'IUDENT' 'TEX'T' (SC28-6808)}

Provides tutorial material not only for the person with some knowledge of compister programming but also for the novice who knows little or nothing about data processing.

\section{P'OR'TABLE PROGRAMS FOR PARALLEL, PROCESSORS (0-(03-014404-3)}

Describes a set of $\mathrm{C}$ tools developed at ANL to explore issues of program performance and portability on a fairly broad range of parallel processors. These tools crablo usors, with lltlo oxtra planning or support, to write programs that easily exploit new machines. They have been Installed on a wide variely of machines, and the authors encourage their use on most of the new systems coming to the market.

\section{POS'TSCRIP'I LANGUAGE REILRENCE MANUAL (0.201.18127.4)}

Is a comprohensive handbonk for programmers interested in interfacing existing applications programs to gencrate PostScript filos or in creating applications in the PostScript language itself. This document bogins with a discussion of basic ldeas underlying PostScript and continues with a comprehensive presentation of the language, its graphics, and its font facilities. An addlthonal chapter contains the semantics of every PostScript operator, organized in dictionary format. This documont concludes with four appendixes, including one that provides dotalled programming information for the Apple LaserWriter. This document is a companion volume to the PostScript Language I'worial and Cookbook (0-201-10179)-3). This document supersedes the PostScript Language Reference Manual (0-201-10174-2),

\section{POS'TSCRIP'T LANGUAGE TU'T'ORIAL AND COOKBOOK (0-201-10179-3)}

Outlines the features and capabilities of the PostScript language and shows practloal ways to create uscful PostScript programs, PostScript is a revolutionary device-independent page description language that has become the industry standard for printing high-quality Integrated text and graphles. Using annotated examples and stort programs, the tutorial section provides a step-by-step guided tour of PostScript, highlighting the qualities that make it a unique and powerful language. The cookbook section offers a collection of some of the more useful techniques and precedures avallable to PostScript programmers. This document is a companion volume to the Postscript Language Reference Manual (0)-201-18127-4).

\section{A PRACITICAL GUIDE T'O UNIX SYSTTEM V $(0-8053 \cdots(0243-3)$}

Explains how to use Unix System V, Release 2, from your terminal. This document uses tutorial examples that show you what you will see on your terminal 
screon ench stop of the way; it takes you from logging on your systom, through writing vomplox shall pro. grams, to system administration. This decoumont assumos that tho render has somo computor exportenco but lltilo or no oxportonice wlth tho Unix systom. How. over, more expertenced Unix systom users will find the later chapters and Part It to bo usoful souroos of infor. mation on subjects such as Sholl programming and systom aciministration. Part II is a "roloronco gulde" to 64 Unix utllitios.

\section{PRODUCING MICROFICHIL OU'TPU'T A'T ANL.} (I'M 373)

Is a combined usor's gulde and reforonoo manual for tho use of microfictio for printed sutput at ANL's Contral IBM Computing Complex, It documonts the produclion of microfiche through a dedicated virtual machine under tho IBM VM control program that was phased in during January 1981 to replace tho microliche support that had lecen provided through the Asymmetric Mulliprocessing Systom (ASP) modifications, This document is a replacement for and makes obsolote Produc:Ing Misroffiche Output at Argonne's Central Computing Facillty (Techuncal Momorandum 26()). This document also supersedos the sections on writing tape datascts to fiche in Displaying Massive PrimImage Filess Via ITP (Technical Momorandum 258). 'This document contains instructions for using micro. fiche from CMS, Wylbur, and batch. 'The discussion of batch job usage of microfiche conforms to MVS/JES3 rules and conventions. This documont summarizes the most significant differences botween the old and now microfiche systems. A July 1981 addendum to this document describes the improved lupe-to-fiche factlly that allows users to submil automatically tape-to-ficho requests from MVT and MVS batch jobs. The new 'TPFICHE command allows the writing of any dataset from a multiple-file tape to microfiche and the writing of multiple-tape standard-fabeled datasels 10 microfiche.

\section{PROGRAMMER'S IN'TRODUC'TION 'TO IBM SYSTIEM/360) ASSWMBLER LANGUAGL: S'TUDIN'T' 'TLX'T' (SC20).1646)}

Is an introduction for students to System/36) Assembler Language coding. It provides many examples of short programs shown in assembled form. In simple, non-technical terms, this document discusses some olomentary programming tochulgues and the specilic
Instructions Illustruted in tho programs, Included is a briol' roviow of rolovant system/360) concepts and desoriptlons of solectod assomblor language linstructhons for arithmotio, logloul, and branching oporations, This decumont includes a discusston onn standard (fixed-point), decimal, and floallng-point aridumetic. This documont also includes an elomontary introducthon to assemblor language and tho assomblor program and chaptors on baso reghstor addressing and on pron. gram linkage and rolocutton. 'Tho cooling of many othor common programming lochnigues (such as the uso of branchos, loops, and counters) is domonstruted, but not oovered in dotall. In genomil, the resudar will find that the program examples are gulte simple at the boginning of each ohapter or major subject divlsion and become progrossivoly moro complex. Students should have accoss to two IBM decumonts: principless of Operation and the assembler specificution dexument for ono of the Sysicm/3o) operaling systems. (All publicallons and their form numbers are listed at the ended of the Profice.) Students should uiso bo familiar with fundumontal concepts of data processing and the baste oporathe princlples of System/36), 'Two IBM prom

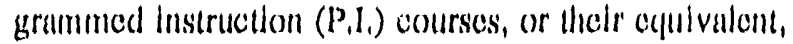
are prerecgutsito to a full understunding of this student toxt: Computing Systom Fundamentals and Introduction to System/360). Students who aro not onrolled in a comprehensive programming courso will find the P,I. book Fundesmentals of Programming a valuable guldo to problom analysls and program flowcharting.

\section{PROLOG PROCIRAMMING FOR AR'TIFICIAL, IN'TELLI(IENCL, SLCOND EDITION} (0)-2(1)1-416(6)(6.9)

Is for students of Prolog and Artificial Intelligence. Prolog is a programming langange contered on a small set of basic mechanisms (including pattern matching, tree-based data structuring, and intomatic backtrackIng). This document Introduces the Prolog langunge and shows how Prolog programs are developed, It also demonstrates the power of Prolog applied in some cen. tral areas of Artificial Intelligence (including problem solving and heuristic search, expert systems, game playing, and pattern-directed systems). The reader should have a basic general knowledge of computers, but no knowledge of Artificial Intelligence is necessary. This document supersedos Prolog Programming for Artiflcial Intelligence, first Edition (()-2()1-14224-4), 


\section{RACF GIENERAL, INHORMA'TION MANUAL, ((IC)28.10722-12)}

Contulns ann overvlew and platming informulion for Versilon I, Rolense \& for MVS and VM ol' the program product Resource Aceess Control Fadilty (RACF),

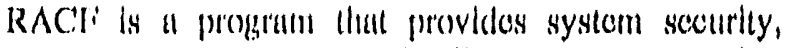
resourco accosss combrol, andillabilley and accountabilly, and adeninistrutive control. Chaptor I discusses the neod for data socurily, provides a basice descriptlon of RACF, and ldonllfies key RACf: fenturos, Chaphlor 2 provedes a hizhinavel introdiction to RACF installation plannling, Clanuler 3 describes RACF functions (including RACF gonorulizallon, RACF.CICS/VS internction, and RACF-IMS/VS intoraction), Chaptor 4 explatis how to use tho RACF commands, ISPF panols, exils, opuloms, and tools to imploment and control $R \wedge(T i$, Chupher 5 summarlos the new functions provided in this documenti, Render's should bo fiunillar will MVS or VM. 'I'this doximent suporsedes tho

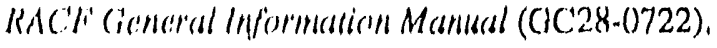

\section{RAC'F SUMMARY FOR CIEN'RAL IBM COMPU'IJIRS' A'T' ANL, (ANL/I'M 478)}

Is a guick relerence for using IBM Rescource Access Conternl fiacility ( $\mathrm{B} / \mathrm{Cl}$ ) soltware that conbles IBM compuner users lo protect their data in accord with their needs. This document supersedes the Cost Center RAC' delministrator's Cimide' (ANL./I'M 42I),

\section{RACI/MVS (IWNERAL, USTER COMMAND) RELFIRLNCLI, CARD (SX28-(06)9-(15)}

Describes the symux of the Resource Access Control lincility (RACF) communds. It is for use by RACF. defined user's who are responsible for crealing, updatIng, or maintaining the prolites for users, groups, direct necess storige device (DASD) datasots, and general resources on the RACF datased.

\section{RACIV/YM (GINNIRAL, USLR COMMAND RLFIERLNCE CARD) (SX22-0008-2)}

Deseribes commonly used commands and operands. for a complete explanation of all commands and operands, seo the RACF Commond Languaga Reference.

\section{RICCOMMISNDICD DOCUMEN'TA'IION FOR COMIU'ILER USLERS A'T ANL., (ANI/'I'M 379, REVISION 3)}

Helpss Argome computer usors solect documentation that will bost filt their Indlvidual needs. We havo rovlsed this doctument extonsively 10 reflect recont ungrades and othor changes in sorvices. Chaptor 1 oxplains how to use this dexument to soled decuments and how to obtain thom from tho CT'D Document Distribution Counter, Chapter 2 contains a table that catoggorizos availablo publications. Chapter 3 gives desoriptions of the cinlino DOCUMEN'I command for CMS, the VAX, and tho Sun workstation. Chaptor 4 llsts publications by subject. Chaptor 5 contains abstracts for each publication, all arranged alphabetically, Chaptor 6 doscribes additional publications contalning bibliographites and mastor indoxes that the usor may find useful. Tho appondix identifles avaltable computer systems, appllicallons, languages, and librarles. T'hls document supersedes Recomunended Dociumentation for Computer Users' al ANL (ANL/TM 379, REVISION 2).

\section{REDUCL' USER'S MANUAL.}

Provides the user with a description of the algebraic programming system REDUCE. The capabilitics of this system lnclude (1) expansion and ordering of polynomials and ratlonal fincitons, (2) substitutions and pattern makching in a widc varlely of forms, (3) antomatic and user-controlled simplification of cxpressions, (4) calculations with symbolic matrices, (5) arbiltrary precision integer and real arithmetic, (6) fucillties for defining new functions and extending program syntax, (7) analytic differentiation and integration, (8) fuctorization of polynomials, and (9) Dirac matrix calculations of interest to high encrgy physicists.

\section{REFERINCE CARD IOR THE ONLINE MA'TERIALS CATALOG}

Describes the Materials Catalog and how to use it through the Customer Information Control System (CICS). This Reference C'ard contains a section on accessing the online calalog through CICS (that is, logging on and off procedures from different networks) and instructions on seven options available to the user: responding to prompts, identifying a catalog code, searching for an $\mathrm{itcm}$, recuesting an expanded item description, scrolling through the catalog, printing a calalog listing, and exiting from the catalog. 


\section{REFWRENCL' CARD FOR USING 'THE COMPUTER CALLBACK SERVICE}

Describes tho Computer Callback Service that allows offilto users in Illinols (with aren codes of $312,7(08$, or 815) 10 aecess onsite computors without incurring large personal costs for tolephone charges. Users who want to use Callback must make sure that their hardware and software configuration and telephone service are compatible with Callback requirements, Before trying to usc Callback, usors should read this Reference Card for full Instructions. This Reference Card supersedes the Juno 1987 Reference Card.

\section{REFERENCL MANUAL FOR THE IBM 3800) PRIN'TING SUIBSYS'TEM (GA26-1635)}

Explains the function and features of the IBM $38(0)$ Printing Subsystem relating to channol command, sense byles, error detection, recovery, and recording. In addition, specific information and examples are given of copy modilication and control and graphic character modilication. This document is for system analysts, installation managers, system programmors, and other personnel requiring IBM $38(0)$ reference information.

\section{RESOURCE ACCESS CONTROL FACILITY (RACF) COMMAND LANGUAGE REFERENCE, VERSION 1, RELEASE 9 (SC28-0733-11)}

Describes the syntax and the functions of the commands for Version 1, Relcase 9 of the Resource Access Control Facility (RACF), Program Number 5740)-XXH, This document is for RACF-defined users who are responsible for creating, updating, or maintaining the profiles for users, groups, clatasets, and general resources on the RACF database on MVS or VM systoms. This document supersedes the Resource Access Control Fasility (RACF) Command Language Reference (SC28-()733-9).

\section{RETRIEVING AND ANALYZING AUTOMATED MA'TERIALS/PAYABLLS SYSTEM DATA AT ANL (ANL/TM 408)}

Defines the process by which the user can obtain specific procurement information from a Statistical Analysis System (SAS) database. The reader will need no specific knowledge of this database but will need some knowledge of the procurement process, of batch submission procedures for simple jobs that execute SAS, and of SAS, In Chapter 3 wo provide samplo programs for obtaining selected data.

\section{RITTRIEVING AND ANAL YZING COMPU'TLR USAGE ACCOUN'TING DATA A'T ANL (ANL/TM 4()2, REVISION 1)}

Describes the set of tools CTD provides to enuble users to retrieve and analyze data from the monthly database containing computor usage information. A combination of commorclal software and locally writter programs generate the databasc. The programs process records of the myrlad tasks that occur during the dally course of data processing operations. The dambase kcops records for every transaction to ensure proper accounting of all debits and credits. 'This database is the same source of information CTD uses to bill customers for services rendered. This doxument supersedes Retrieving and Anclyzing Computer Usagge Accounling Dala al ANL (ANL/TM 4()2).

\section{SAS COMPANION FOR THE CMS ENVIRONMENT, VERSION 6, FIRST EDITION $(1.55544 .399 .0)$}

Provides both tutorial and reference information about the SAS System under CMS. It provides host-specific details to supplement the information in SAS Language and Procedures: Usage, Version 6, First Edition (1-55544-371-()); SAS Language: Reference, Version 6. First Edition (1-55544-381-8); and the SAS Procedures Guide, Version 6, Third Edition (1-55544-378-8). Parts 1 and 2 show you how to perform common lasks and explain how you can use the SAS System under CMS to accomplish these tasks. Parts 3, 4, and 5 provide complete descriptions of all the features of the SAS System that have behavior specific to CMS, but do not attempt to teach you how to use the software. This document is for SAS System users who have had some experience with the SAS System, but not necessarily under CMS. This document assumes that users can log on and log off CMS and that they are familiar with basic CMS commands.

\section{SAS COMPANION FOR THE CMS OPERATING SYSTEM (1-55544-0(11-0)}

Supplements the SAS User's Guide (Version 5) and other SAS documents that describe SAS coneepts, language, and procedures. In CMS, SAS is evoked through an interface program that creates a special 
environment. The interface interprets CMS/SAS options, allocates files, provides supervisor services, and supports auxiliary processes. This document describes and illustrates the use of SAS in the CMS environment. First, read the SAS Introductory Guide for a quick explanation of SAS capabilities. Then consult this document for examples and technical information on using SAS in CMS. This docur int does not contain a complete discussion of CMS. The sample terminal sessions use important CMS commands. Instead of learning everything about CMS before you start, just look up the recommended commands as you need them. Figures of SAS input and output show SAS usage with both full screen and ASCII terminals. Keep in mind that this book covers unmodified CMS; be alert for local enhancements.

\section{SAS COMPANION FOR THE MVS ENVIRONMENT, VERSION 6, FIRST EDITION (1-55544-398-2)}

Provides both tutorial and reference information about the SAS System under MVS. Parts 1 and 2 show you how to perform common tasks and explain how you can accomplish these tasks by using the SAS System under MVS. Parts 3, 4, and 5 provide complete descriptions of all the features of the SAS System that have host-specific behavior but do not teach you how to use the software. This document provides hostspecific details to supplement the information in SAS Language and Procedures: Usage, Version 6. First Edition (1-55544-371-0); SAS Language: Reference, Version 6. First Edition (1-55544-381-8); and the SAS Procedures Guide, Version 6, Third Edition (1-55544-378-8).

\section{SAS COMPANION FOR THE VMS ENVIRONMENT, VERSION 6, FIRST EDITION (1-55544-392-3)}

Provides both usage and reference information about the SAS System under VMS. Parts 1 and 2 show you how to perform common tasks and explain how you can use the SAS System under VMS to accomplish these tasks. Parts 3,4 , and 5 provide complete descriptions of all the features of the SAS System that have system-dependent behavior, but do not attempt to teach you how to use the software. This document is for SAS System users who have had some experience with the SAS System, but not necessarily under VMS. This document supersedes the SAS Companion for the VMS Operating System. 1986 Edition (0-917382-98-6).

\section{SAS GUIDE TO MACRO PROCESSING, VERSION 6, SECOND EDITION (1-55544-382-6)}

Provides complete documentation on portable features of the Release 6.06 SAS macro facility used with base SAS software. In this context, portable includes features that work the same across all host operating systems. (In contrast, host-specific features are those that are meaningful only under specific host operating systems or that work differently under different host operating systems.) This document includes an introductory tutorial, reference material, a chapter of examples that provide usage material, and a glossary. This document is for users who either have previous experience with the SAS System or who have at least an intermediate level of experience with another programming language and who understand the fundamentals of programming logic. This document supersedes the SAS Guide to Macro Processing, Version 5 Edition and SAS Technical Report P-175, Changes and Enhancements to the SAS System, Release 5.18, under OS and CMS.

\section{SAS GUIDE TO THE MICRO-TO-HOST LINK, VERSION 6 EDITION (1-55544-069.X)}

Documents the use of the Statistical Analysis System (SAS) micro-to-host link. The SAS link is a feature of base SAS software that allows communication between a PC SAS session on a microcomputer and a SAS session on another larger computer--the host. The SAS link gives you access to the files and SAS software on the host computer at the same time that you are running a PC SAS session. This document describes the enhanced 6.02 release of the SAS tink and Release 5.16 of the host SAS systems.

\section{SAS INTRODUCTORY GUIDE FOR PERSONAL COMPUTERS, RELEASE 6.03 (1-55544-095-9)}

Describes the Statistical Analysis System (SAS) as it is used on personal computers. As one easy-to-use system, it provides all the tools necessary for data analysis: information storage and retrieval, data modification and programming, report writing, statistical analysis, and file handling. This document's goal is to help you learn enough about SAS so that you can use it to get the answers you need quickly. This document assumes you are familiar with your personal computer and the basic functions of its various keys. 


\section{SAS LANGUAGE AND PROCEDURES: INTRODUCTION, VERSION 6, FIRST EDITION (1-55544-410-5)}

Provides basic introductory material about base Statistical Analysis System (SAS) software, Release 6.06. This document describes base SAS software and explains how to access, analyze, manage, and present data in various formats. This document is for those who want to learn how to use base SAS software and who are new SAS users.

\section{SAS LANGUAGE AND PROCEDURES: SYNTAX, VERSION 6, FIRST EDITION (1-55544-400-8)}

Provides a quick but complete reference to the syntax of the portable base Statistical Analysis System (SAS) software. In this context, portable includes features that work the same across all host operating systems. This document is not meant to replace reference guides for base SAS software. This document is for users who either have previous experience in using the SAS System or who have at least an intermediate level of experience with another programming language and understand the fundamentals of programming logic.

\section{SAS LANGUAGE AND PROCEDURES: USAGE, VERSION 6, FIRST EDITION (1-55544-371-0)}

Provides instructions and examples for accomplishing the primary tasks you need to complete in your daily use of the Statistical Analysis System (SAS). This document is a tutorial for base SAS software beginning with Rclease 6.06. This document focuses on common tasks and explains the simplest way of accomplishing them. This document is for users who have not used the SAS System but have some computer programming experience and know the fundamentals of programming logic, who have an introductory-level knowledge of the SAS System, or who are experienced SAS users who want to learn new tasks.

\section{SAS LANGUAGE GUIDE OR PERSONAL COMPUTERS, RELEASE 6.03 (1-55544-099-1)}

Contains the fundamentals of the Statistical Analysis System (SAS): an introduction to the DATA and PROC steps, the syntax and use of SAS statements, and system files and options. The SAS system is a software system for data analysis; it provides tools for information storage and retrieval, data modification and programming, report writing, statistical analysis, and file handling. This document is organized to reflect the structure of the SAS language for both new and experienced SAS users. The information in this document is arranged according to where it can be used in a SAS program--in a DATA step, a PROC step, or anywhere.

\section{SAS LANGUAGE: REFERENCE, VERSION 6, FIRST EDITION (1-55544-381-8)}

Provides complete reference information for all portable language features in Release 6.06 base Statistical Analysis System (SAS) software. In this context, portable includes features that work the same across all host operating systems. (In contrast, host-specific features are those that are meaningful only under specific host operating systems or that work differently under different host operating systems.) This document includes all aspects of base SAS software that are not procedures. This document is for users who either have previous experience with the SAS System or who have at least an intermediate level of experience with another programming language and understand the fundamentals of programming logic. This document supersedes the SAS User's Guide: Basics, Version 5 Edition (0-917382-65-X); SAS Technical Report $P .146$, Changes and Enhancements to the Version 5 SAS System; SAS Technical Report P-168, Changes and Enhancements to the Version 5 SAS System under VMS; SAS Technical Report P-175, Changes and Enhancements to the SAS System, Release 5.18, under OS and CMS; SAS Technical Report P.182, Changes and Enhancements to the SAS System, Release 5.18, under VMS; and SAS Technical Report P-183, Changes and Enhancements to the SAS System, Release 5.18, under AOSIVS and PRIMOS.

\section{SAS PROCEDURES GUINE FOR PERSONAL COMPUTERS, RELEASE 6.03 (1-55544-089-4)}

Describes all procedures in the Version 6 base Statistical Analysis System (SAS) software. There are four categories of procedures: elementary statistics, reporting, scoring, and utilities. Chapters 1 through 4 introduce the four categorics of procedures; the introductory section for each group briefly describes the procedures available for that category and what they do. The remaining chapters describe the procedures individually. The statements and syntax necessary io run tacit procedure are presented in a uniform format throughout this document. 


\section{SAS PROCEDURES GUIDE, VERSION 6, THIRD EDITION (1-55544-378-8)}

Provides complete reference information for all procedures in Release 6.06 base Statistical Analysis System (SAS) software. This document is for users who have not used the SAS System but who have some computer programming experience and know the fundamentals of programming logic and for experienced SAS users.

\section{SAS PROGRAMMER'S GUIDE FOR PL/I, VERSION 5 EDITION (0-917382-91-9)}

Describes in detail the process of writing a procedure that interfaces with the SAS system. This document also describes how to write SAS functions, SAS CALL routines, SAS formats and informats, and SAS/IML library routines.

\section{SAS TECHNICAL REPORT P-201: CHANGES AND ENHANCEMENTS TO SAS SOFTWARE FOR PERSONAL COMPUTERS, RELEASE 6.04 (1-55544-393-1)}

Documents the upgrade to base Statistical Analysis System (SAS) software and SAS/Graph software for personal computers in Release 6.04. This report should be used as a supplement to Release 6.03 documentation.

\section{SAS TECHNICAL REPOR'T P-204: SAS SOFTWARE CHANGES AND ENHANCEMENTS, RELEASE 6.06 (1-55544-409-1)}

Provides reference information about changes from Relcase 5.18 to Release 6.06 Statistical Analysis System (SAS) software. However, it does not list any host-specific changes that are included in Release 6.06 SAS software. It is also not intended as a primary source of production information. This document is for users who are already familiar with Version 5 software and want an overvicw of enhancements available in Version 6 and for new SAS software users who are interested in some of the new features included in Release 6.06.

\section{SAS USER'S GUIDE: BASICS, VERSION 5 EDITION (0-917382-65-X)}

Contains the fundamental information needed to use the Statistical Analysis System (SAS): (1) an introduction to DATA and PROC steps; (2) the syntax and use of SAS statements; (3) macros; (4) system options; and (5) procedures for descriptive statistics, report writing, and utilities. This document is the first of two volumes that are the primary documentation for the SAS system. Many SAS users need only the data processing, summarizing, and reporting features of the system without further statistical analysis. This two-volume format provides a choice of one or both documents, depending on whether you are using SAS primarily for data processing or for statistics.

\section{SAS USER'S GUIDE: STATISTICS, VERSION 5 EDITION (0-917382-66-8)}

Complements the SAS User's Guide: Basics, which provides primary documentation for those using the Statistical Analysis System (SAS) primarily for data processing. This document contains advanced statistical procedures in the areas of regression, analysis of variance, categorical data analysis, multivariate analysis, discriminant analysis, clustering, scoring, and the MATRIX language.

\section{SAS/ETS USER'S GUIDE, VERSION 6, FIRST EDITION (1-55544-325-7)}

Documents the SAS/ETS software, including (1) time series extracting, data management, and plotting; (2) forecasting and time series extraction; (3) modeling and econometrics; and (4) financial reporting. The SAS/ETS procedures require prior knowledge of the SAS system and elementary statistics. This document supersedes the SAS/ETS User's Guide (0-917382-62-5). See SAS/PC ETS Software, Version 6.04

\section{SAS/FSP USER'S GUIDE, VERSION 5 EDITION (0-917382-67-6)}

Documents the SAS/FSP software. The software provides interactive, menu-driven facilities for data entry, editing, and retrieval. This SAS/FSP release contains the following procedures: (1) PROC FSCALC for using the capabilities of a full-range electronic spreadsheet, (2) PROC FSCON for converting existing letter 
and screen datasets into catalogs for use with Version 5 SAS/FSP procedures, (3) PROC FSBROWSE for displaying observations in a SAS dataset, (4) PROC FSEDIT for entering and editing tata in a SAS dataset,

(5) PROC FSLETTER for editin $b$ and sending letters,

(6) PROC FSLIST to look at listing files, and

(7) PROC FSPRINT to look at SAS datasets a full screen mode as a table of rows and columns.

\section{SAS/GRAPH GUIDE TO HARDWARE INTERFACES, VERSION 5 EDITION} $(0-917382-69-2)$

Describes the device drivers compatible with Version 5 SAS/GRAPH software and explains in detail how to set up and use graphics hardware at a given site. SAS/GRAPH users should also read the SAS/GRAPII User's Guide, Version 5 Edition, which is the primary documentation for SAS/GRAPH software.

\section{SAS/GRAPH SOFTWARE: INTRODUCTION, VERSION 6, FIRST EDITION (1-55544-395-8)}

Provides basic introductory material about SAS/Graph software, Relcase 6.06, and demonstrates how to produce simple charts, maps, plots, and text slides. This document is for those who want or need to learn how to use SAS/Graph software and who consider themselves new users. See SAS/PC Graph Software, Release 6.04 .

\section{SAS/GRAPH SOFTWARE: REFERENCE, VERSION 6, FIRST EDITION (1-55544-379-6)}

Is a two-volume set that provides reference information about all facilities, procedures, statements, and options that you can use with SAS/Graph software, Release 6.06. These documents do not attempt to teach you how to use the software. These documents are for users experienced in using SAS software. Users should understand the concepts of programming in the SAS language and should have an idea of the tasks they want to perform with SAS/Graph software. This document supersedes the SAS/GRAPII User's Guide, Version 5 Edition (0-917382-68-4).

\section{SAS/GRAPH USER'S GUIDE, VERSION 5 EDITION (0-917382-68-4)}

Defines a computer graphics system for producing color plots, charts, maps, and other displays on screens and plotters. SAS/GRAPH programs are actually SAS procedures. If you are familiar with the SAS procedures CHART and PLOT, you will find that your CHART and PLOT statements can be used for SAS/GRAPH by changing the procedure name to GCHART or GPLOT, although additional options to produce colorful and patterned displays are available. All SAS retrieval, data management, analysis, and other capabilities may be used with SAS/GRAPH. Data values are put into SAS datasets before SAS/GRAPH procedures use them. Chapter 1 serves as an introduction to SAS/GRAPH. Chapter 2, "Global Statements," describes the statements used to set up the SAS/GRAPH environment. Next, in alphabctical order, are the SAS/GRAPH procedures GCHART, GCONTOUR, GMAP, GOPTIONS, GPLOT, GPRINT, GPROJECT, GREDUCE, GREPLAY, GSETUP, GSLIDE, and G3D. The chapters describing GCHART, GCONTOUR, GMAP, GPLOT, and G3D begin by showing several examples using color and other SAS/GRAPH options. The appendixes show the various character fonts available through SAS/GRAPH, describe the SAS/GRAPH functions, give a short description of some of the hardware devices compatible with SAS/GRAPH, and describc the map datasets available with SAS/GRAPH.

\section{SAS/PC BASE SOFTWARE, RELEASE 6.04}

Provides tools for information storage and retrieval, data modification and programming, report writing, statistical analysis, and file handling. You can add tools for graphics, forecasting, data entry, and interfaces to other databases to the all-purpose base SAS software. For use on IBM or IBM-compatible personal computers.

\section{SAS/PC ETS SOFTWARE, RELEASE 6.04}

Provides tools for (1) time series extracting, data management, and plotting; (2) forecasting and time serics extraction; (3) modeling and econometrics; and (4) financial reporting. For use on IBM or IBMcompatible personal computers. See the SAS/ETS User's Guide, Version 6, First Edition (1 55544-325-7). 


\section{SAS/PC GRAPH SOFTWARE, RELEASE 6.04}

Provides tools for producing simple charts, maps, plots, and text slides. See SAS/Graph Software: Introduction, Version 6, First Edition (1-55544-395-8).

\section{SAS/PC STAT SOFTWARE, RELEASE 6.04}

Is a wide range of statistical applications for data analysis and estimation (including regression, analysis of variance, categorical, multivariate, discriminant, and scoring). The SAS/PC Stat software must be used with the Base SAS/PC software. For use on IBM or IBMcompatible personal computers.

\section{SAS/STA'T USER'S GUIDE, RELEASE 6.03 EDITION (1-55544-088-6)}

Documents all of the procedures available in Release 6.03 of SAS/STAT software. Chapters 1 through 8 provide an overview and introduce you to the eight groups of procedures in SAS/STAT software. Chapter 9 contains detailed information about the four types of estimable functions used by many regression and analysis-of-variance procedures. The next 26 chapters describe individual SAS/STAT procedures in alphabetical order. This document supersedes the SAS/STAT Guide for Personal Computers, Version 6 Edition.

\section{SAS/STAT USER'S GUIDE, VERSION 6 , FOURTH EDITION (1-55544-376-1)}

Is a two-volume set that documents the procedures available with Release 6.06 of SAS/STAT software. Volume 1 contains the introductory chapters and documents thic ACECLUS through FREQ procedures. Volume 2 documents the GLM through VARCOMP procedures. This document assists data analysts who use SAS/STAT software. This document assumes you are familiar with basic Statistical Analysis System (SAS) concepts (such as creating SAS datasets with the DATA step and manipulating SAS datascts with the procedures in base SAS software). For mainframe and minicomputer sites using Release 6.06 software, these documents supersede the SAS User's Guide: Statistics, Version 5 Edition (0-917382-66-8) and sections on the statistical procedures in the following technical reports: SAS Technical Report P-146, Changes and Enhancements to the Version 5 SAS System; SAS Technical Report P.I68. Changes and Enhancements to the Version 5 SAS System under VMS; SAS Technical Report P-175. Changes and Enhancements to the SAS System,
Release 5.18, under OS and CMS; SAS Technical Report P-182, Changes and Enhancements to the SAS System, Release 5.18, under VMS; and SAS Technical Report P.183, Changes and Enhancements to the SAS System, Release 5.18, under AOS/VS and PRIMOS. For microcomputers and Unix workstations, these documents supersede the SAS/STAT Guide for Personal Computers, Version 6 Edition (1-55544-064-9) and the SAS/STAT User's Guide, Release 6.03 Edition (1-55544-088-6). If you are using the SAS System with PC DOS, continue to use the SAS/STAT User's Guide, Release 6.03 Edition.

\section{SCIENTIFIC CHART DESIGN: COMPUTER-GENERATED CHARTS USING TELL-A-GRAF}

Is for people who want to produce their own scientific charts with Tellagraf. This alternative document to the Tellagraf User's Manual presents a series of major design elements in scientific charts. It also provides examples to highlight alternative choices and to show the Tellagraf specifications you can use to make the different charts.

\section{SCRIP'T REFERENCE CARD}

Describes the format of the physical page output by Script. It further lists Script control words by function as well as alphabetically. It also gives an Options summary, a Set Symbol and Macro Summary, and a Symbol Attribute Function Summary.

\section{SECURITY GUIDELINES FOR MICROCOMPUTERS AND WORD PROCESSORS (DOE/MA-0181)}

Is issued by the Office of ADP Management as a reference to security for microcomputers and word processors. It provides techniques that you should consider when processing DOE sensitive unclassified data on a microcomputer or word processor.

\section{SECURITY OF PERSONAL COMPUTER SYSTEMS: A MANAGEMENT GUIDE (NBS500-120)}

Describes management and technical security considerations associated with the use of personal computer systems. The primary objective is to identify and dis- 
cuss several areas of potential vulnerability and associated protective measures. The document discusses physical and environmental protection, system and data access control, integrity of software and data, back-up and contingency planning, auditability, and communications protection. It also includes a general plan for the management of personal computer information security. Appendixes provide references to additional information, a self-audit checklist, and a guide to security products for personal computers.

\section{SEGMENT LOADER (SEGLDR) AND LD REFERENCE MANUAL (SR-0066 6.0)}

Describes the Segment Loader's (SEGLDR's) operation, method of code exccution, common block use, and common block assignment. SEGLDR operates on Cray Y-MP, Cray X-MP EA, Cray X-MP, and Cray-2 computer systems running under the Unix-Based Cray Operating System (UNICOS). The glossary explains SEGLDR terminology, but this document assumes that the reader understands overlays and is familiar with loaders. Additionally, this document documents the UNICOS command ld that is an interface to the segment loader in the style of traditional Unix system loaders. This document supersedes the Segment Loader (SEGLDR) and ld Reference Manual (SR-0066 E).

\section{SENSITIVE UNCLASSIFIED COMPUTER SECURITY PROGRAM COMPLIANCE REVIEW GUIDELINES (DOE/MA-0188/1)}

Is an Office of ADP Management reference for planning or performing a sensitive unclassified computer security program compliance revicw in accord with the provisions of DOE Order 1360.2: "Computer Security Program for Unclassified Computer Systems." This document explains how to establish effective computer security and to achieve the levels of computer security appropriate to individual DOE ADP sites.

\section{SINGLE LINE TELEPHONE (STE) USER GUIDE (ANL/TM 470)}

Explains how to use the standard single line telephone with the Argonne telephone system. The single line telephone is STE (standard telephone equipment).

\section{SLAC WYLBUR TUTORIAL}

Is available with minor changes and an addendum reflecting the local conventions. We reprint this document because it is an excellent introduction to Wylbur for new users. Argonne used Stanford Linear Accelerator Center (SLAC) Wylbur from May 1976 until we replaced it in June 1980 with On-Line Business :ystems (OBS) Wylbur. Because the layout of this document does not allow for full revision, we have made minor changes in text and blanked out some sections that would now confuse inexperienced users. As you gain experience with Wylbur, you will want to try the other features documented in the OBS Wylbur Reference Manual and in the online HELP information.

\section{SOLUTION OF THE GENERAL NONLINEAR PROGRAMMING PROBLEM WITH SUBROUTINE VMCON (ANL-80-64)}

Describes how to solve the general nonlinear programming problem with a subroutine called VMCON. VMCON uses an algorithm proposed and first implemented by M.J.D. Powell, based on the earlier work of S-P Han. Powell's algorithm solves a sequence of positive definitc quadratic programming subprograms. Each solution determines a direction in which a onerimensional minimization is performed. The current implementation contains extensive in-line documentation; an interface subroutine, VMCON1, with a simplified calling sequence; and print options to aid the user in interpreting results.

\section{SPECIFICATIONS FOR THE PBX-BASED DIGITAL TELECOMMUNICATIONS SYSTEM (ANL/TM 418)}

Contains the ANL requirements for a Private Branch Exchange (PBX)-based digital telecommunications system (DTS). This system serves Argonne National Laboratory and the Department of Energy at the Argonne, Illinois, site, where it has replaced the Centrex telephone service provided by Illinois Bell. The new system provides cost-effective telephone service and has greatly improved voice and data communications. 


\section{STORES STANDING ORDERS AT ANL (ANL/TM 441, REVISION 1)}

Is for the Inventory Planners and the Material Handlers within Materials and Services, for the Buyers in Procurement, and for the Voucher Payment Clerks within Accounts Payable, already trained in the Stock Tracker and the Automated Materials/Payables systems but not in Stores Standing Orders. This document limits itself to the procedures specific to Stores Standing Orders at ANL and to the computer software built for that subset of procurement functions. This document therefore supplements existing procedure and policy guides for the Stock Tracker and the Automated Materials/ Payables systems. Chapter 1 defines a Stores Standing Order at ANL and summarizes briefly the steps to establish such an agreement, to purchase materials under the agrecment, and to monitor the agrecment through its lifetime. Chapters 2 through 10 then describe in detail cach of the steps and provide instructions for performing specific tasks. Chapter 11 defines some differences between Stores Stunding Orders and the Argonne Materials Order System (AMOS). This document supersedes Stores Standing Orders at ANL (ANL/TM 441).

\section{SUGI SUPPLEMENTAL LIBRARY USER'S GUIDE, VERSION 5 EDITION (1-55544-000-2)}

Documents 15 new procedures written and contributed by Statistical Analysis System (SAS) users to the SUGI Supplemental Library. It also updates or repeats documentation for 35 procedures and 11 SAS functions included in earlier editions of this document.

\section{SUMMARY OF ANSYS REVISION 4.4A NEW FEATURES}

Lists the new fealures in ANSYS Revision 4.4A.

\section{SURVEY OF ANL, ORGANIZATION PLANS FOR WORD PROCESSORS, PERSONAL COMPUTERS, WORKSTATIONS, AND ASSOCIATED SOFTWARE (ANI/TM 459, REVISION 3)}

Supplements information in both the ANL Statement of Site Strategy for Computing Workstations (ANL/TM 458) and the ANL Site Response for the DOE Information Technology Resources Long-Range Plan (ANL/TM 466). This Survey documents each ANL ôtganization's plans for office automation, identifies appropriate planners and other contact people in those organizations, and promotes the sharing of this information among those people making plans for organizations and decisions about office automation. This document supersedes Survey of ANL Organization Plans for Word Processors, Personal Computers, Workstations, and Associated Software (ANL/TM 459, REVISION 2).

\section{SYMBOLIC MACHINE INSTRUCTIONS REFERENCE MANUAL (SR-0085)}

Provides reference information on Cray X-MP and Cray-1 computer systems symbolic machine instructions and is to be used as a reference with the CAL Assembler Version 2. The CAL Assembler Version 2 allows you to express symbolically all hardware functions of a Cray X-MP and Cray-1 computer system. This detailed and precise level of programming is especially helpful for tailoring programs to the architecture of a Cray mainframe and for writing programs requiring code optimized to the hardware.

\section{TABLES DESIGNER'S GUIDE}

Is a companion volume to the TABLES User's Guide. Both are Integrated Software Systems Corporation (ISSCO) documents that describe how to use ISSCO's TABLES software. This document is for users who wish to custom design their own tables or to modify the standard formats extensively.

\section{TABLES USER'S GUIDE}

Is a companion volume to the TABLES Designer's Guide. Both are Integrated Software Systems Corporation (ISSCO) documents that describe how to use ISSCO's TABLES software. This document uses a step-by-step "hands-on" session to explain how to make basic tables by using the standard TABLES formats.

\section{TCP/IP AND OSI NETWORK USER'S GUIDE (SG-2009 6.0)}

Intruduces network users to the communication capabilitics available with the Transmission Control Protocol/Internet Protocol (TCP/IP) and the Open Systems Interconnection (OSI) on Cray Rescarch computcr systems running the Unix-Based Cray Operating System (UNICOS). This document describes the 
UNICOS networking commands that you can execute at a Cray Research system to communicate with a remote system. Readers should be familiar with either Unix or UNICOS and with the networking products installed on their workstations or computer terminals.

\section{TELEBIT T2500 ASYNCHRONOUS MODEM USER'S GUIDE WITH OPERA'TING INSTRUCTIONS (ANL/TM 492)}

Helps users of the Telebit T2500 dial-up modem use the modem to access ANL computing systems from offsitc. These instructions supplement the Telebit T2500 Reference Manual and the Telebit T2500 Fast Start Guide, which the Telebit Corporation supplics with the modem.

\section{TELLAGRAF/PINPOINT USER'S MANUAL (PIN-UG-0985)}

Is the Computer Associates (CA) guide to using Pinpoint, CA's interactive enhancement to Tellagraf. Pinpoint lets you change the position and size of chart items on your Tellagraf chart without issuing any Tcllagraf commands. You simply point to the Pinpoint screen and enter your instructions. Before attempting to learn Pinpoint, you should have a basic knowledge of Tellagraf and Tellagraf commands. Chapter 1 of this document is an overview that introduces Tellagraf and Pinpoint. Chapter 2 reviews Tellagraf commands and command format. Chapter 3 discusses devices used with Pinpoint. Chapter 4 is a step-by-step tutorial for beginners on how to use Pinpoint. Chapter 5 provides details about Pinpoint use, including particulars about each menu option and each chart item you can change.

\section{TELLAGRAF/PINPOINT WORKSTATION REFERENCE CARD DEC VT241 VT240 HEWLETT-PACKARD 262326472648 (PIN-QR-1085-DEC)}

Is one of several Integrated Software Systems Corporation (ISSCO) cards containing information on how to use specific terminals when you are doing interactive graphics with Tellagraf/Pinpoint. Each card also includes a brief review of Pinpoint menu options. For more detailed information on Tellagraf/Pinpoint, consult the TellagraffPinpoint User's Guide. This Reference Card contains specific information on how to use DEC VT241 and VT240 terminals and HewlettPackard 2623, 2647, and 2648 terminals.

\section{TELLAGRAF/PINPOINT WORKSTATION \\ REFERENCE CARD IBM 3179 G 3270 PC/G 3270 PC/GX (PIN-QR-1085-3179)}

Is one of several Integrated Software Systems Corporation (ISSCO) cards containing information on how to use specific terminals when you are doing interactive graphics with Tellagraf/Pinpoint. Each card also includes a brief review of Pinpoint menu options. For more detailed information on Tellagraf/Pinpoint, consult the TellagraffPinpoint User's Guide. This Card contains specific information on how to use IBM 3179 $\mathrm{G}$ terminals and $3270 \mathrm{PC} / \mathrm{G}$ and $3270 \mathrm{PC} / \mathrm{GX}$ computcrs.

\section{TELLAGRAF/PINPOINT WORKSTATION REFERENCE CARD IBM 3279 3270 PC (PIN-(QR-1085-3279)}

Is one of several Integrated Software Systems Corporation (ISSCO) cards containing information on how to use specific terminals when you are doing interactive graphics with Tellagraf/Pinpoint. Each card also includes a brief review of Pinpoint menu options. For more detailed information on Tellagraf/Pinpoint, consult the TellagraftPinpoint User's Guide. This Card contains specific information on how to use IBM 3279 terminals and $3270 \mathrm{PC}$ computers.

\section{TELLAGRAF/PINPOIN'T WORKSTATION REFERENCE CARD TEKTRONIX 40104012 40134014401540164051405240544081 (PIN-QR-1085-4010)}

Is one of several Integrated Software Systems Corporation (ISSCO) cards containing information on how to use specific terminals when you are doing interactive graphics with Tellagraf/Pinpoint. Each card also includes a brief review of Pinpoint menu options. For more detailed information on Tellagraf/Pinpoint, consult the TellagraflPinpoint User's Guide. This Card contains specific information on how to use Tektronix $4010,4012,4013,4014,4015,4016,4051,4052,4054$, and 4081 terminals. 


\section{TELLAGRAF/PINPOINT WORKSTATION REFERENCE CARD TEKTRONIX 4027 (PIN-QR-1085-4027)}

Is one of several Integrated Software Systems Corporation (ISSCO) cards containing information on how to use specific terminals when you are doing interactive graphics with Tcllagraf/Pinpoint. Each card also includes a brief review of Pinpoint menu options. For more detailed information on Tellagraf/Pinpoint, con. sult the TellagraftPinpoint User's Guide. This Card contains specific information on how to use Tektronix 4027 terminals.

\section{TELLAGRAF/PINIOOIN'T WORKSTATION REFERFNCE CARD TEKTRONIX 41044105 4106 (PIN-QR-0386-4104)}

Is one of several Integrated Software Systems Corporation (ISSCO) cards containing information on how to use specific terminals when you are doing interactive graphics with Tellagraf/Pinpoint. Each card also includes a brief review of Pinpoint menu options. For more detailed information on Tellagrai/Pinpoint, consult the TellagraftPinpoint User's Guide. This Card contains specilic information on how to use Tektronix $41(44,4105$, and 4106 terminals.

\section{TELLAGRAF/PINPOINT WORKSTATION REFERENCE CARD TEKTRONIX 41064107 4109 (PIN-QR-1085-4106)}

Is one of several Integrated Software Systems Corporation (ISSCO) cards containing information on how to use specific terminals when you are doing interactive graphics with Tellagraf/Pinpoint. Each card also includes a bricf review of Pinpoint menu options. For more detailed information on Tellagraf/Pinpoint, consult the TellagraffPinpoint User's (iuide. This Card contains specific information on how to use Tektronix 4106,4107 , and $410 \%$ terminals.

\section{TELLAGRAF/PINPOINT WORKSTATION REFERENCE CARD TEKTRONIX 41124113 411441154116412541284129 (PIN-QR-1085-4112)}

Is one of several Integrated Software Systems Corporation (ISSCO) cards containing information on how to use specific torminals when you are doing interactive graphics with Tellagraf/Pinpoint. Each card also includes a brief review of Pinpoint menu options. For more detailed information on Tellagraf/Pinpoint, consult the I'ellagraffPinpoint User's Guide. This Card contains specific information on how to use Tcktronix $4112,4113,4114,4115,4116,4125,4128$, and 4129 terminals.

\section{THE TEXBOOK (0-201-13448.9)}

Is a handbook about $\mathrm{TeX}$, a typesetting system for the creation of books, especially ones that contain a lot of mathematics. By preparing a manuscript in TeX format, you are telling a computer exactly how to transform the manuscript into pages of high typographic quality; yet you won't need to do much more work than would be involved if you were simply typing the manuscript on ordinary paper. This document is for people who have never used TeX before; it explains everything you need to know about $\mathrm{TeX}$.

\section{THTB A'T ANL (TM 281)}

Describes THTB (Transient Heal Transfer, Version B), proprietary code written originally by General Electric for the IBM 704. It is capable of analyzing general three-dimensional heat transfer systems using a finite difference method. The basic function of the program is to set up the general heat balance equation for each node point, to compute the terms that apply from the input given, and to solve the resulting set of equations by the Gauss-Seidel method for the central temperatures of the nodes. The process is repeated for each time increment by using the temperatures from the previous time increment as approximations to the new temperatures.

\section{TN3270 FOR THE MACINTOSH REFERENCE GUIDE, VERSION 2.0}

Explains how to use tn 3270 for the Apple Macintosh computer with a Transmission Control Protocol/ Internet Protocol (TCP/IP) network connection to emulate an IBM 3270 terminal. Features of the cmulation include extended highlighting, APL, and IBM 3179-compatible graphics. For an Apple Macintosh II or other Apple Macintosh with a large screen, the emulation program accommodates different screen formats. In IBM CMS, tn3270 acts as a file transfer protocol (FTP) server for file transfer and maintains file transfer a part of a terminal session. Although $\operatorname{tn} 3270$ is primarly an IBM 3270 emulation program, it also accommodates line mode Telnet connections. This feature 
allows tn 3270 to operate with hosts that establish a line mode connection before entering 3270 mode as well as line mode only hosts. When operating in line mode, tn 3270 emulates a DEC VT52 terminal.

\section{TSO MESSAGES (GC28-1310-4)}

Explains TSO and TSO Extensions messages and provides some diagnostic information for people who debug programs.

\section{UNICOS CDBX DEBUGGER USER'S GUIDE (SG-2094 6.0)}

Describes the characteristics and capabilities of the CDBX debugger, which is avallable to users of the Cray. It explains the ways in which you can work with the CDBX debugger and provides examples of commonly used commands. This document is for programmers and others who run applications written in CAL, CFT, CFT2, CFT $77, \mathrm{C}$, and Pascal. Readers should have a working knowledge of either the Unix-Based Cray Operating System (UNICOS) or the Unix operating system, plus the language in which their application programs are written.

\section{UNICOS CDBX SYMBOLIC DEBUGGER REFERENCE MANUAL (SR-2091 6.0)}

Describes the CDBX debugger available to users of the Unix-Based Cray Operating System (UNICOS) running on Cray systems. This document is for programmers and others who run applications written in Cray Asscmbler Language (CAL), Fortran, C, and Pascal. Readers should have a working knowledge of either the UNICOS or Unix operating system, plus the language in which their application programs are written. This document supersedes the UNICOS CDBX Symbolic Debugger Reference Manual (SR-2091 5.0),

\section{UNICOS CFT REFERENCE CARD (SQ-0022)}

Provides the line format for the CFT command. This Reference Card also lists the value ranges represented in the Cray computer system, the compiler options and directives, the Fortran language statement syntax and parameter definitions, the Fortran-callable subprograms, the generic and specific intrinsic function names, the DO-loop features inhibiting vectorization, and the ASCIl character set.

\section{UNICOS CFT77 REFERENCE CARD (SQ-0138 B)}

Describes the features of the Fortran 77 compiler. This Reference Card lists the format of the CFT command, compiler options and functions, Fortran language statement syntax, intrinsic functions, and available compiler directives.

\section{UNICOS OVER VIEW FOR USERS (SG-2052-A)}

Provides an overview of the Unix-Based Cray Operating System (UNICOS) and incorporates the new features of the UNICOS 4.0 release. This document provides introductory information for anyone using UNICOS for the first time. Readers do not need extensive prior knowledge of UNICOS, Unix, or the Cray Operating System (COS). Topics include capabilitics provided by UNICOS, important features of UNICOS, the basic structure of the UNICOS operating system, and a description of what jobs and output will look like. This document does not provide a detailed description of UNICOS, nor does it describe all of the opc:ating system's capabilities.

\section{UNICOS PERFORMANCE UTILITIES REFERENCE MANUAL (SR-2040 6.0)}

Describes performance utilities that run on the Cray Y-MP, Cray X-MP EA, Cray X-MP, and Cray-2 computer systems under the Unix-Based Cray Operating System (UNICOS). This document describes the means of invoking these utilities, their options, and their output. This document supersedes the UNICOS Performance Utilities Reference Manual (SR-2040 A).

\section{UNICOS PRIMER (SG-2010 6.0)}

Provides introductory information on the Unix-Based Cray Operating System (UNICOS) running on Cray systems. (Other Cray Research, Inc. publications cover specific aspects of the operating system.) This document assumes that the reader has some experience in programming and is familiar with general program. ming concepts (such as looping, files, filc editing and editors, and conditional branches). This document supersedes the UNICOS Primer (SG-2010 C). 


\section{UNICOS SHELL AND VARIABLE RLADY REFERLNCE (SQ-2060)}

Provides a quick reforence for the Unix-Based Cray Operating System (UNICOS) uscrs who want to customize their environuents by using the $\mathrm{C}$ or Bourne shell. The handbook covers the following topics: idenufying and changing sholls, operating in the Bourne shell, operating in the $\mathrm{C}$ shell, and environment varlables for use with either shell. Readers should have a general knowledge of shells and shell scripts.

\section{UNICOS SUPPOR'T TOOLS GUIDE (SG-2016 6.0)}

Describes some of the more sophisticated software tools available to users of the Cray. Readers should have a working knowledge of the $C$ programming language and either the Unix operating system or the Unix-Based Cray Operating System (UNICOS),

\section{UNICOS SYMBOLIC DEBUGGING PACKAGE REFERENCE MANUAL (SR*0112 C)}

Describes the operation of the Symbolic Debugging Package. This package has the following analytical tools: DEBUG (a postmortem core analyzer), SYMDUMP and SYMDEBUG (snapshot dump library routines), DRD (Dyramic Runtime Debugger), and DDA (Dynamic Dump Analy'zer). These tools allow you to debug programs cither interactively or in batch. The tools in the Symbolic Debugging Package operate on the Cray Y-MP, the Cray-2, the Cray X-MP, and the Cray-1 computer systems under the Cray Operating System (COS) and the Unix-Based Cray Operating System (UNICOS). This document describes how to use the symbolic: debugging tools under UNICOS. Readers stiould be familiar with both UNICOS and the programming language in which their programs are written.

\section{UNICOS SYSTEM CALLS REFERENCE MANUAL (SR-2012 5.0)}

Describes the system calls available on the Cray-2, Cray Y-MP, Cray X-MP EA, Cray X-MP, and Cray-1 computer systems and supplements the information contained in other documents in the Unix-Based Cray Operating System (UNICOS) documentation set. This revision documents changes for the UNICOS 5,0 release. Readers should have a working knowledge of either the UNICOS or Unix operating system. This document supersedes the UNICOS System Calls Reference Manual (SR-2()12).

\section{UNICOS 'TAPE SUBSYSTIEM USER'S GUIDE} (S( $\$ 20516.0)$

Describes the characteristics and capabilities of the Unix-Based Cray Operating System (UNICOS) tape subsystem, which was developed for use with Cray Y-MP, Cray X-MP EA, and Cray X-MP computer systems that have an I/O Subsystem (IOS) or Cray-2 com. puter systems that have a Cray-2 Tape Controller (CTC). This document explains how to work with the tape subsystom and provides many examples of commonly used commands. Readers should have a working knowledge of elther UNICOS or Unix.

\section{UNICOS USER COMMANDS READY REFERENCE MANUAL (SQ-2056 6.0)}

Provides condensed descriptions of the Unix-Based Cray Operating System (UNICOS) user commands available on all Cray Rescarch systems. This docilment supersedes the UNICOS User Commands Ready Reference (SQ-2056 5.0).

\section{UNICOS USER COMMANDS REFERENCE MANUAL (SR-2011 6.0)}

Is a two-volume document that documents the UnixBased Cray Operating System (UNICOS) Release 6.) rurning on the Cray Y-MP, Cray X-MP EA, Cray $\mathrm{X}-\mathrm{MP}$, and Cray-2 systems. This document provides descriptions of commands and application programs for system users of UNICOS. It supplements the information in the other documents in the UNICOS documentation set. This document describes programs that are invoked directly by the user or by command language procedures. Many commands reside in the directory /bin (for binary programs). Programs can also reside in /usr/bin, /usr/ucb (those commands ported from the Fourth Berkeley Software Distribution), /lib, and /usr/lib. The /bin, /usr/bin, and /usr/ ucb directories are searched automatically by the command interpreter called the shell. You must change the path (or specify the path on the command line) if you want to use a command in /etc, /lib, or /usr/lib. The commands for which this is necessary are specified with their full pathnames in the SYNOPSIS section on the man page. This document is a reference document for UNICOS programmers and assumes that the reader 
has a working knowledgo of olthor the UNICOS or Unix oporathes systom. Thils dooumont suporsodes the UNICOS User Commands Refaranca Manual (SR-20115.(0),

\section{UNICOS VI REIERLNCL CARD (SQ-2054)}

Summarlzes commonly-used vl commands in a pocketsize roforence card.

\section{UNICOS X WINDOW SYSITEM RENIERLNCLE MANUAL $($ SR-2101 6.0)}

Provides information on features of the $\mathrm{X}$ Window System that are specific to Cray Rescarch, Inc, (CRI) applications. Readers should have a working knowledge of the $\mathrm{X}$ Window Systom and olther the UnixBused Cray Operating System (UNICOS) or the Unix opcrating system.

\section{UNIVERSITY OF CHICAGO AGRLEMEN'TS WITH PERSONAL COMPU'IER VIENIDORS}

Contains the latest available lists of personal computer discounts avallable through the Iniversity of Chicago to Argonne employees for both personal and Laboratory purchases. This list is usually updated monthly.

\section{UPDATE REFERENCE MANUAL (SR-(0)13 K)}

Describes UPDATE, a program that modifies, edits, and updates source language programs operating under the Unix-Based Cray Operating System (UNICOS) and the Cray Operating System (COS) on the Cray Y-MP, Cray X-MP EA, and Cray X-MP computer systems and under UNICOS on Cray-2 systems. Readers should be familiar with the general features of COS or UNICOS.

\section{USER GUIDE FOR MINPACK-1 (ANL-80-74)}

Describes MINPACK-1, a package of Fortran subprograms for the numerical solution of systems of nonlinear equations and nonlinear least squares problems. This report provides an overview of the algorithms and software in the package and includes the documentation and program listings.

\section{USINC LINEMMODIE SUPPOR'T WI'TH THII' PROHUSSIONAL OFIICIS SYS'TLM (SII20*68(12)}

Provides roforenco Information and step-by-step) Instructions for using the Professlonal Offico Systom (PROFS) on a lino-modo typo torminal, Only thosso lasks doscribod in this book aro avallablo for lino-modo uscrs of PROFS. Chapter 1 covers things you nood 10 know, no matter what ank you dom-such things as how PROFS stores information and how your PROFS administrator can holp you. Chaptors 2 through 7 match the tasks that you can porform by using PROFS and a linomodo typo terminal. These who do not already have tho IBM bindor for PROFS documonts (SB3()-(2480) should order one with this document,

\section{USING MS.DOS KERMIT: CONNEC'TING YOUR PC 'TO THEA WLEC'TRONIC WORLD)} $(1.55558-(1) 82-3)$

Describes the MS-DOS Kermit Version 3.11 for the IBM PC, PS/2, and compatibles and includes a $51 / 4$-Inch diskette. This document suporsedes $U$ ising MS-DOS Kermit (1-5.5558-(248-3),

\section{USING MS-DOS $5(0 \cdot 88(1) 22-668 \cdot 4)$}

Offers DOS Version 5 Users a source of information to halp them organize their work with the PC more effectively, to make their hardware respond more cfficient. ly, and to provide a resource of answers to questions about the capabilities of DOS. This document is written for PC users who need a tutorlal reference to DOS.

\section{USING SYSTTEM TRANSMITTALS AT ANL (ANL/T'M 437)}

Describes the modified version of the transmittal system as it functions in CTD. This document defines the functional requirements that the transmittal system satisfies, describes the interface for transmiltal users, and provides installation details. Since the Software Library is the designated repository for copies of all system transmittals, this document complements the information in The Computing and Telecommunications Division Software Library (ANL/TM 463). 


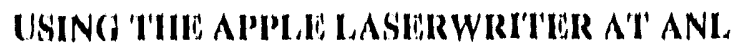
(ANL./'I'M 452)

Ofllors holpful Information for any Argonno compulor uscrs with CMS, MVS balch, or VAX/VMS acoounts (or any porsonal computor uscris with accoss to the 3Com notwork) who noed to print guality text or graphles on tho Applo Latior Writter. Thils document describos how to uso the Apple LaserWrilor with Watorloo Script, Integrated Soltwaro Systoms Corporation graphics soltware (l,o, Cuechart, 'I'ellagraf', and Dlss. pla), and somo Applo Macintosh graphics software (o. E., MacDraw and MacPalnt)) to produce (quallty formatled text, graphlics, or a morger of both on printed pages. The documont does not altompt to describe the use of the Apple LasorWriter with other applications (c.g., personal computer products), though some information on the handling of PostScript files may be valid for other applications.

\section{USIN( THW CEN'TRAX, VAX 8700 COMPIITER A'I' ANL (ANL/I'M 455, RLVISIION 2)}

Is a reference document for users of the Argonne National Lationatory central VAX 870)( computer. It provides inlormation on how to use the computers in the central VAX cluster and how to use the VAX/VMS system for gencral interactive and batch use. This document also describs:s the software and graphics packag. es available on the central VAX 87(0), T'wo appendixes explain the ("I'D policy for user accounts and how to use the VAX supercomputer gateway to the Argone Cray X-MP/14. Realders will need some knowledge of VMS. When appropriate, wis edition refers to informution in other documents; it does not ordinarily duplicate information availables in vendor documents. This document supersedes Using the Central VAX 8700) Computer at ANL. (ANL/TM 455, REVISION 1).

\section{USING 'THIE FINANCIAL MANAGEMEN'T SYS'TLEM A'T ANL, (ANL/T'M 446)}

Describes Argonne's Financial Management System (FMS) and how to use it. FMS is a reporting system that provides access to Argonne's cost and budget data. It also allows users to produce reports, to determine financial codes, to gencrale graphs of financial data, to define and use work breakdown structure, to aceess the Project Management System IV (PMS IV) Cost Processor, and to send financial dala to other ANL computer systems. 'This document consists of five chapters, two appendixes, and an index. Chapter 1 discusses the subsystems of FMS and illustrates the relationshlp between FMS and other ANL financial systems, Chaptors 2 and 3 provide instruction on gainIng FMS authorlzation, accessing FMS (lata, and kecpling abreast of FMS systom changes and data updates. Chaptor 4 discussos cach FMS function in dotall so that you can use these functions to gain information, run a report, manage a saved report, run batch jobs, and understand miscellaneous coxles. Chaptor 5 explains how to obtain assistance wlih FMS. Appendix A provides additional information on rolated documontation. Appendix B is a glossary of torms.

\section{USING 'THE HYDRA PRO'TOCOL CONVER'T'ER IOR IISM FULL SCREEN 'TERMINAL EMULATION A'T ANL (ANL/TM 457, REVISION 1)}

Explains how to lake advantage of IBM full screen convenience features with the more popular terminals and Iorminal emulation programs by using the Hydra Protocol Converter (extonsion 2-3270). Hydra full screen emulation is avaliable for all the central IBM computer interactive services (CMS, MVS Wylbur, CICS, and MVS TSO), (Many terminals and terminal cmulation programs emulate DEC VT1(X) or Televideo 950) terminals, which are documented in Chapters 2 and 3, respectively.) This revision reflects vendor improvements to the Hydra Prolocol Converter hardware and changes in vendor-supplied terminal emulations. Revisions in the Hydra terminal selection menu reguired changes in the RELLAY Gold scripts described in Chap. ter 4, "Using RELAY Gold on the IBM PC for Full Screen Emulation." Chapter 5, "Using MS-Kermit on the IBM PC for Full Screen Emulation," covers the new MS-Kermit version 2.31, which reculires new key mapping files and minor changes to cmulation key assignments. A revised Chapter 6, "Using MacKermit on the Macintosh for Full Screen Emulation," includes revisions for the new $0,9(40)$ Kermit version. This document supersedes Using the Hydra Protocol Converter for IBM Full Screen Terminal Emulation at ANL (ANL/T'M 457).

\section{USING THE PROFESSIONAI, OFFICE SYSTEM (SH20-6797.01)}

Is a reference guide for anyone who uses the Professional Office System (PROFS). Readers should have a general knowledge of PROFS. This document super" sedes Using the Projessional Office System (SH20-6797). 


\section{USING THE PURCHASE INFORMA'TION REPOR'TING SYSTEM A'T ANL (ANL/TM 419)}

Supplomonts and to somo extont supersodos Retrieving and Analyzing Automated Materials/Payables System Data at ANL (ANL/TM 408). The Purchase Information Roporting System (PIRS) is a major component of tho Pillot Project. PIRS allows for the timely transfor of information from the Automated Matorials Payables Systom (AMPS) on the Howlotl-Packard 300() computers to the mainframe computers in the contral computing complex. This document describos how to access and understand this information through an intorface that is easy to understand and use.

\section{VAX DOCUMENTA'TION BINDER (1.5 INCH) (99.07858.01)}

Is a 1.5 -inch binder for VAX documentation; it contains no written material.

\section{VAX DOCUMENTATION BINDER (2.5 INCH) (99.07862-01)}

Is a 2.5 -inch binder for VAX documentation; it contains no written material.

\section{VAX EDT REFERENCE MANUAL} (AA-LA16A-TE)

Serves as a reference source for the EDT interactive text editor. It is for all users of EDT. This document supersedes the VAX EDT Reference Manual (AA-Z300A-TE).

\section{VAX FORTRAN LANGUAGE REFERENCE MANUAL (AA-D034E-TE)}

Is a reference manual that describes the VAX Fortran language for VMS. The revision is part of a twovolume set for VAX Fortran Version 5.0 users. Volumc 1, VAX Fiortran Language Reference Manual, describes how to use the VAX/VMS system. Readers should have a basic understanding of the Fortran language and the VMS system. This document supersedes Programming in VAX Fortran (AA-D034D-TE).

\section{VAX FOR'TRAN USER'S MANUAL (AA-D035E-T'E)}

Explains how to create, complle, link, execute, and debug VAX Fortran programs on a VMS operaling system. This revision is part of a two-volume set for VAX Fortran Version 5.(), Volume 2, VAX Fortran Language Reference Manual, describes the VAX Fortran language for VMS. Readers should have a basic understanding of the Fortran language and the VMS system. This dexument supersedes the VAX Fortran User's Guide (AA-D()35D-TE).

\section{VIRUSCAN/CL.ISAN-UP FOR IBM PC V8GB}

Is a $51 / 4$-inch diskette with the current release of Viruscan and Clean-up, a disinfectant program for the IBM Personal Computer. These programs can detect and correct known viruses (such as Jerusalem B, Fish, Fish6, and Yankec Doodle). CTD requests that you not make copies of these programs for others to use, because our license is limited to $1(0)$ coples of each of these programs. Copies of this diskette are avallable at the Document Distribution Counter. CTD is keeping a master list of all persons to whom these programs have been distributed. This V84 diskette supersedes the V80 diskctte.

\section{VMS DCL DICTIONARY: PART I, VERSION 5.4 (AA.PBK5A-TE)}

Is Part I of a two-part document. It contains Digital Command Language (DCL) commands with the letters A to $\mathrm{M}$, as well as lexical functions. The remaining commands are in Part II. This document is for all users of the VMS operating system.

\section{VMS DCL DICTIONARY: PART II, VERSION 5.4 (AA-PBK6A-'TE)}

Is Part II of a two-part document. It contains DCL commands with the letters $\mathrm{N}$ to $\mathrm{Z}$. The remaining commands and the lexical functions are in Part I. This document is for all users of the VMS operating system.

\section{VMS DEBUGGER MANUAL (AA-LA5913-TE)}

Explains the features of the VMS Debugger for programmers in high-level and assembly languages. Readers should be programmers at all levels of exporience. 


\section{VMS DECWINDOWS USER'S GUIDE} (AA-MG18A-TE)

Is designed to help users explore and use VMS DECwindows. Readers should have a general knowledge of VMS DECwindows.

\section{VMS USER'S MANUAL, VERSION 5.4 (AA-LA98B-TE)}

Provides an overview of the VMS operating system. This document is for all users of the VMS operating system. This document has two major parts. Chapters 1 through 13 describe VMS concepts and procedures users need to perform basic computing tasks. The Reference Section contains the following VMS user reference information: Digital Command Language (DCL) comrats: DIGITAL Standard Runoff (DSR) command, w EDT editor, Extensible VAX Editor (EVE) commands, MAIL, and VMS Sort/Merge. This document supersedes the VMS General User's Manual, Version 5.0, which had superseded the VAX/VMS User's Manual (AI-Y517A-TE).

\section{VMSCHEDULE USER'S MANUAL}

Provides an overview of the VM Software program product $\checkmark$ MSCHEDULE, along with detailed descriptions of the available VMSCHEDULE subcommands. VMSCHEDULE allows CMS users to schedule programs, execs, and commands for automatic logon and execution. Chapter 1 provides a general introduction to VMSCHEDULE. Chapter 2 illustrates a practical application of each subcommand through a variety of examples. Chapter 3 contuins the format of each subcommand and detailed descriptions of related options and parameters. Four appendixes offer more specitic advice.

\section{VMUSP: VMUPASS-THROUGH FACILITY GUIDE AND REFERENCE (SC2+5208)}

Is for anyone whose VM/Pass-Through responsibilities include planning, system programming, installing the VM/Pass-Through Facility and operating or using the VM/Pass-Through Facility. This document assumes that people who have responsibilities for planning, installing, and operating Pass-Thrcugh have some knowledge abou: teleprocessing systems. Appendix A contains VM/Pass-Through messages.

\section{VM/SYSTEM PRODUCT: EDITOR XEDIT PROGRAM FUNCTION KEY TEMPLATE FOR IBM DISPLAY TERMINALS (SX24-5123)}

Provides information on the Program Function Key Template. This XEDIT Program Function Key template is to be placed above the program function keys of an IBM 3278/3279 display terminal. It is an easy reference to the uses of the program function keys.

\section{VM/SYSTEM PRODUCT: GETTING STARTED WITH THE PROFESSIONAL OFFICE SYSTEM (SH20-5602)}

Is for anyone who is new to PROFS. It shows you how PROFS works by leading you through some basic tasks with examples and step-by-step instructions.

\section{VOLUME 1: UNICOS FORTRAN LIBRARY REFERENCE MANUAL (SR-2079 6.0)}

Describes Fortran-callatlis subprograms and routines available to users of the Cray. It supplements the information contained in other documents in the UnixBased Cray Operating System (UNICOS) documentation set. This document is a reference manual for application and system programmers. Readers should have a working kno'wledge of either the UNICOS or Unix operating system.

\section{VOLUME 3: UNICOS MATH AND SCIENTIFIC LIBRARY REFERENCE MANUAL (SR-2081 6.0)}

Documents the math and scientific library routines available to users of the Unix-Based Cray Operating System (UNICOS), Release 6.0. It supplements the information contained in the other documents in the UNICOS documentation set. Readers should trave a working knowledge of either the UNICOS C Unix operating system. This document supersedes the UNICOS Mash and Scientific Library Reference Manual (SR-2081 5.0).

\section{VS B ASIC (CMS) REFERENCE SUMMARY (SX28-6386)}

Is a digest of the VS Basic language and the requirements for its use under the CMS system. It assumes you are familiar with the language and system details described in VS Basic Language and the VS Basic CMS Terminal User's Guide. 


\section{VS FORTRAN VERSION 2 INTERACTIVE DEBUG GUIDE AND REFERENCE, RELEASE 3 (SC26-4223-2)}

Describes how to use VS Fortran Version 2 Interactive Debug to monitor and test the running of VS Fortran programs. Scientists, engineers, other professionals, and students who use VS Fortran for engineering and scientific problem solving can use this book easily and effectively. Part One discusses how to use Interactive Debug, and Part Two describes all Interactive Debug commands.

\section{VS PASCAL APPLICATION PROGRAMMER GUIDE (SC26-4319)}

Is a guide to the use of the VS Pascal compiler. It explains how to compile, linkedit, execute, and debug VS Pascal programs. This document does not describe the VS Pascal language, which is explained in the VS Pascal Language Reference.

\section{VS PASCAL LANGUAGE REFERENCE (SC26-4320)}

Is the reference document to the VS Pascal Application Programmer Guide (SC26-4319). This document assumes that you are already familiar with Pascal and programming in a high-level programming language.

\section{VSAM PRIMER AND REFERENCE (G320-5774)}

Is a guide and a reference source for the person using the Virtual Storage Access Method (VSAM). The objectives of this document are (1) to give the new VSAM user the information required to understand, evaluate, and use VSAM properly and (2) to clarify VSAM functions for current VSAM application programmers who will be working with VSAM. The practical straightforward approach adopted should dispel much of the apparent complexity sometimes associated with VSAM.

\section{THE WAITE GROUP'S NEW C PRIMER PLUS $(0-672-22687-1)$}

Is a complete, easy-to-understand introduction to the $\mathrm{C}$ programming language for both the novice and experienced programmer. This document covers (1) the fundamentals of programming (including principles of structurid code, step-wise refinement, and top-down design); (2) C itself (operators, expressions, stiviements, input/output, functions, and arrays and pointers); and (3) how to use special library functions, storage classes, string functions, data forms, and other advanced $\mathrm{C}$ skills. This document supersedes Programming in $C$ $(0-672-48420-X)$.

\section{WATERLOO SCRIPT AND A POSTSCRIPT PRINTER}

Describes the current level of compatibility in Water100 Script with any printer that has a PostScript interpretive language interface. The University of Waterloo has developed this level of compatibility from specifications documented in the PostScript Language Manual from Adobe Systems Incorporated. All information and functions of this interface should apply to every PostScript printer, no matter what printing hardware is used in the implementation. This document assumes that readers know how to Script formatted documents to such devices as fixed pitch terminals and line printers and are familiar with such concepts as control words, macro calls with operands, text records, and multiple Script input files. This document discusses the benefits of using Script with a new device having different capabilities. It covers the new information required, the assumptions to be made, and the output that can be generated, both from the point of view of the end user and those who are primarily concerned about how the device is driven.

\section{WATERLOO SCRIPT GML USER'S GUIDE}

Describes Generalized Markup Language (GML) for use with Waterloo Script as a text formatter. GML provides a convenient way of annotating text for computerized formatting. With GML, you identify each component of a document with a "tag" in your file. For example, the "GDOC" tag indicates the start of a document, and the "eGDOC" tag indicates the end of the document. Several document layouts are available through GML, depending on the "GMLxxxxx" filc that you imbed at the beginning of your file (where "xxxxx" is the "guide" format or one of the other prescribed formats).

\section{WATERLOO SCRIPT REFERENCE MANUAL}

Describes Waterloo Script, a text formatter for document composition. Script is used to format an input file containing text and control lines. The file may contain fixed-length or variable-length records or $\mathrm{Wyl}$ - 
bur Edit-format records. Formatting is specified by control lines (lines that begin with a control indicator, normally a period, followed by a two-letter control word). CTD recommends Script with the Waterloo Generalized Markup Language (GML) as formatters for documents produced through the central Argonne computers. GML is a new text formatter that the University of Waterloo has developed to replace Syspub.

\section{WATERLOO SCRIPT USER'S GUIDE}

Describes Waterloo Script, a text formatter for document composition. With just a few Script commands (called "control words"), you can satisfy the formatting requirements of most simple documents, such as office correspondence and small papers. The full power of Script is realized in the preparation of large "structured" documents (such as books, manuals, theses, and rescarch, technical, and instructional publications containing footnotes, tables, figures, indexes, and tables of contents). By using thero-writing features of Script, it is possible to automate the preparation and maintenance of documents and to standardize their layout.

\section{WATERLOO SCRIPT WITH FORMULA PROCESSING}

Explains the new Firmula Processor implemented with Script Version 86.1. Users can now enter mathematical and scientific notation as part of the text with no more difficulty than is normally involved in entering text into a computer file. The Formula Processor will produce composed formulas for any output device (i.e., the Apple LascrWriter) for which Script can (1) generate controls to manipulate a variety of text and symbol fonts in a range of different sizes and (2) place characters at an arbitrary position on the output page. Primarily, this document describes the Formula Processor input language and provides examples that illustrate how the output is produced. This document is for those unfamiliar with sophisticated techniques of printing and without mathematical backgrounds. The input language contains mathematical operators, special symbols, and simple text, all in a form that you can enter from the keyboard of the average terminal. A local addendum covers options available to Argonne Script users.

\section{WATERLOO SYSPUB USER'S GUIDE}

Describes Syspub, a set of Script Macros for preparing and formatting papers, manuals, theses, and various types of research and reference publications. With Waterloo Script, Syspub offers several advantages for computer text-formatting.

\section{WYLBUR USER REFERENCE SUMMARY}

Describes Text Editing commands, Job Maragement commands, SET and SHOW commands, Dataset commands and Execute Programming Functions. This card also describes some Wylbur conventions, definitions, and abbreviations.

\section{$X$ WINDOW SYSTEM USER'S GUIDE (0-937175-14-5)}

Describes window system concepts and the application programs (clients) commonly distributed with Version 11 , Release 4 of X. Because some commercial X systems still reflect X11, Release 3, this book highlights the important differences between the two releases. This document is for both first time and experienced "sers of the X Window System. Part One provides an overview of the $X$ Windnw System and concepts and describes how to use the most important programs available in the $X$ environment. Part Two tells you how to customize $X$ to your liking. Part Three provides Unix-style "man-pages" for each of the $X$ programs or clients. Part Four contains useful reference information.

\section{PRINTING SUBSYSTEM CHARACTER GAUGE (GX35-5012)}

Explains the IBM 3800 printing subsystem character gauge, which is for use together with the IBM 3800 print charts. It is useful in designing actual sizes and form overlays. 


\section{CHAPTER 6}

\section{BIBLIOGRAPHIES AND MASTER INDEXES}

Those who wish to explore reading material outside the scope of this document will find the following list of bibliographies useful.

The following IBM publications contain bibliographies of IBM publications:

IBM System/370 and 4300 Processor Bibliography (GC20-0001-3)

IBM System/370 and 4300 Processors: Bibliography of Industry Systems and Application Programs (GC20-0370-2)

The following IBM publications contain master indexes. A master index is a consolidated index from various publications that directs you to the publications that contain the specific information you are seeking:

IBM Virtual Machine System Product: Library Guide, Glossary, and Master Index (GC19-6207) MVS/370 Data Facility Product: Master Index (GC26-4062)

The following DEC publication contains a bibliography of DEC software documentation:

Overview of VMS Documentation (AA-LA95A-TE)

The following Cray Research, Inc. publication contains a bibliography of Cray documentation:

Cray Research, Inc.: User Publications Catalog (CP-0099) 


\section{APPENDIX}

AVAILABLE APPLICATIONS AT ANL 


\begin{tabular}{|c|c|c|c|c|c|}
\hline \multicolumn{6}{|c|}{$\begin{array}{l}\text { Table } 2 \\
\text { Available Applications at ANL }\end{array}$} \\
\hline Application & IBM CMS & IBM MVS Batch & DEC VAX/VMS & Cray UNICOS & Unix \\
\hline $\begin{array}{l}\text { Artificial } \\
\text { Intelligence }\end{array}$ & & & Prolog & & \\
\hline Communications & $\begin{array}{l}\text { Kermit } \\
\text { Telnet }\end{array}$ & & $\begin{array}{l}\text { Kermit } \\
\text { DECnet } \\
\text { NJE Extension } \\
\text { Telnet }\end{array}$ & Telnet & Telnet \\
\hline $\begin{array}{l}\text { Database \& File } \\
\text { Management }\end{array}$ & $\begin{array}{l}\text { SAS } \\
\text { Inquire }\end{array}$ & $\begin{array}{l}\text { SAS } \\
\text { Inquire }\end{array}$ & $\begin{array}{l}\text { SAS } \\
\text { Datatrieve } \\
\text { DEC CDD }\end{array}$ & & \\
\hline Debugging & TESTFORT & & $\begin{array}{l}\text { Interactive } \\
\text { yn bolic } \\
\text { Debugger }\end{array}$ & DEBUG & $c d b x$ \\
\hline Editors & Xedit & Wylbur & $\begin{array}{l}\text { EDT } \\
\text { TPU (EVE) } \\
\text { LSE }\end{array}$ & $\mathrm{vi}$ & $\begin{array}{l}\text { vi } \\
\text { Gnu-Emacs }\end{array}$ \\
\hline File Transfer & $\begin{array}{l}\text { Kermit } \\
\text { FTP }\end{array}$ & & \begin{tabular}{|l} 
FTP \\
NJE Extension
\end{tabular} & FTP & $\begin{array}{l}\text { Kermit } \\
\text { FTP }\end{array}$ \\
\hline Graphics Software & $\begin{array}{l}\text { Cuechart } \\
\text { Tellagraf } \\
\text { Data Connection } \\
\text { Disspla } \\
\text { SAS/Graph } \\
\text { Speakeasy }\end{array}$ & $\begin{array}{l}\text { Disspla } \\
\text { SAS/Graph }\end{array}$ & $\begin{array}{l}\text { Cliechart } \\
\text { Tullagraf } \\
\text { Data Connection } \\
\text { Disspla } \\
\text { SAS/Graph } \\
\text { DECwindows }\end{array}$ & $\begin{array}{l}\text { Disspla } \\
\mathrm{X} \text { Window }\end{array}$ & $\begin{array}{l}\text { Disspla } \\
\text { X Window }\end{array}$ \\
\hline $\begin{array}{l}\text { Mathematical } \\
\text { Libraries }\end{array}$ & $\begin{array}{l}\text { IMSL } \\
\text { NAG } \\
\text { AMDLIB }\end{array}$ & $\begin{array}{l}\text { IMSL } \\
\text { NAG } \\
\text { AMDLIB }\end{array}$ & $\begin{array}{l}\text { IMSL } \\
\text { NAG } \\
\text { DOE SLATEC }\end{array}$ & $\begin{array}{l}\text { IMSL } \\
\text { NAG } \\
\text { DOE SLATEC }\end{array}$ & \\
\hline $\begin{array}{l}\text { Office } \\
\text { Automation }\end{array}$ & PROFS & & & & \\
\hline $\begin{array}{l}\text { Programming } \\
\text { Languages }\end{array}$ & $\begin{array}{l}\text { Basic } \\
\text { C } \\
\text { Fortran } \\
\text { Pascal } \\
\text { PL/I } \\
\text { Speakcasy }\end{array}$ & $\begin{array}{l}\text { Cobol } \\
\text { Fortran } \\
\text { PL/I } \\
\text { Pascal }\end{array}$ & $\begin{array}{l}\text { Basic } \\
\text { C } \\
\text { Fortran } \\
\text { Pascal } \\
\text { Macro } \\
\text { Prolog }\end{array}$ & $\begin{array}{l}\text { C } \\
\text { Fortran } \\
\text { Pascal }\end{array}$ & $\begin{array}{l}\text { C } \\
\text { Fortran }\end{array}$ \\
\hline
\end{tabular}




\begin{tabular}{|c|c|c|c|c|c|}
\hline & & $\begin{array}{l}\text { Table } 2 \text { (Contin } \\
\text { vailable Applicatio }\end{array}$ & $\begin{array}{l}\text { ued) } \\
\text { ns at ANL }\end{array}$ & & \\
\hline Application & IBM CMS & IBM MVS Batch & DEC VAX/VMS & Cray UNICOS & Unix \\
\hline $\begin{array}{l}\text { Simulation } \\
\text { Packages }\end{array}$ & CSMP III & $\begin{array}{l}\text { CSMP III } \\
\text { THTB }\end{array}$ & & & \\
\hline Sorting \& Merging & $\begin{array}{l}\text { SORT } \\
\text { SORTF } \\
\end{array}$ & DFSORT & & & \\
\hline $\begin{array}{l}\text { Source Code } \\
\text { Maintenance }\end{array}$ & & $\begin{array}{l}\text { Librarian } \\
\text { Historian }\end{array}$ & $\begin{array}{l}\text { DEC CMS } \\
\text { DEC MMS }\end{array}$ & $\begin{array}{l}\text { UPDATE } \\
\text { SCSS } \\
\text { MAKE }\end{array}$ & $\begin{array}{l}\text { update } \\
\text { scss } \\
\text { make }\end{array}$ \\
\hline Spreadsheet & & & DECalc & & \\
\hline Statistical Analysis & $\begin{array}{l}\text { SAS } \\
\text { Spcakeasy } \\
\end{array}$ & \begin{tabular}{|l} 
SAS \\
BMDP \\
\end{tabular} & SAS & & \\
\hline Symbolic Algebra & REDUCE & & DOE-MACSYMA & & \\
\hline $\begin{array}{l}\text { Terminal } \\
\text { Enhancement }\end{array}$ & IBM VM/370 DMS & & \begin{tabular}{|l} 
FMS \\
TDMS \\
\end{tabular} & & $\operatorname{tn} 3270$ \\
\hline Text Processing & Waterloo Script & Waterloo Script & $\begin{array}{l}\text { Mass-11 } \\
\text { LaTeX } \\
\text { TeX } \\
\text { RUNOFF }\end{array}$ & & $\begin{array}{l}\text { LaTeX } \\
\text { TeX } \\
\text { troff }\end{array}$ \\
\hline
\end{tabular}


NOTES 
NO'TES 


\section{NOTES}


NO'TES 
NO'TES 
NOTES 
Recommended Documentation for Computer Users at ANL

ANL/TM 379

\section{NOTES}




\section{NOTES}


NOTES 

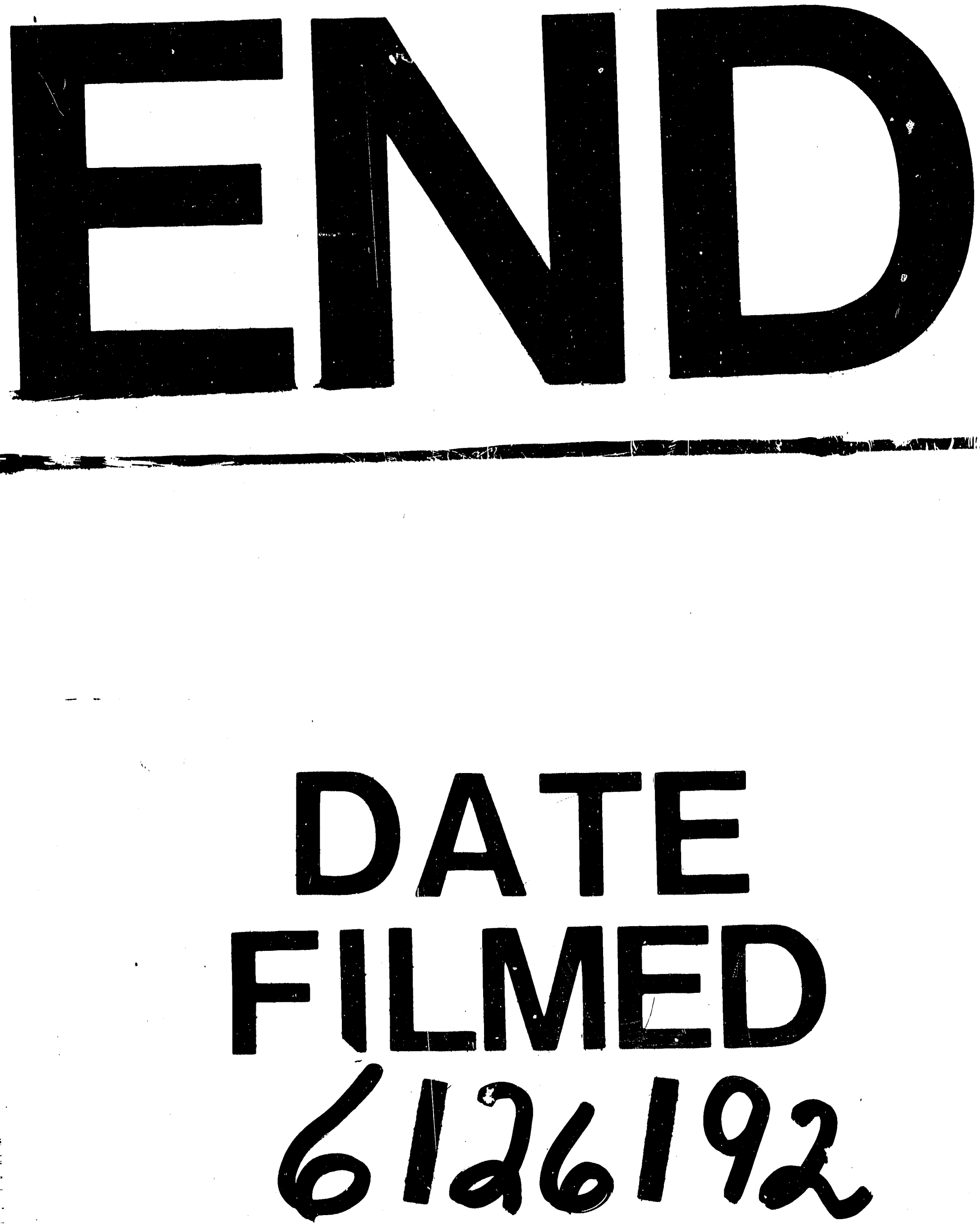
